\title{
Theoretical modeling of microstructured liquids: a simple thermodynamic approach
}

\author{
Matteo Pasquali ${ }^{\mathrm{a}, *, 1}$, L.E. Scriven ${ }^{\mathrm{b}}$ \\ ${ }^{a}$ Department of Chemical Engineering, Rice University, MS 362, 6100 Main Street, Houston, TX 77005, USA \\ b Coating Process Fundamentals Program, Department of Chemical Engineering and Materials Science, \\ University of Minnesota, Minneapolis, MN 55455, USA
}

Received 11 September 2003; received in revised form 19 February 2004

This article is part of a Special Volume containing papers from the 3rd International Workshop on Nonequilibrium Thermodynamics and Complex Fluids

\begin{abstract}
A new method is presented for accounting for microstructural features of flowing complex fluids at the level of mesoscopic, or coarse-grained, models by ensuring compatibility with macroscopic and continuum thermodynamics and classical transport phenomena. In this method, the microscopic state of the liquid is described by variables that are local expectation values of microscopic features. The hypothesis of local thermodynamic equilibrium is extended to include information on the microscopic state, i.e., the energy of the liquid is assumed to depend on the entropy, specific volume, and microscopic variables. For compatibility with classical transport phenomena, the microscopic variables are taken to be extensive variables (per unit mass or volume), which obey convection-diffusion-generation equations. Restrictions on the constitutive laws of the diffusive fluxes and generation terms are derived by separating dissipation by transport (caused by gradients in the derivatives of the energy with respect to the state variables) and by relaxation (caused by non-equilibrated microscopic processes like polymer chain stretching and orientation), and by applying isotropy. When applied to unentangled, isothermal, non-diffusing polymer solutions, the equations developed according to the new method recover those developed by the Generalized Bracket [J. Non-Newtonian Fluid Mech. 23 (1987) 271; A.N. Beris, B.J. Edwards, Thermodynamics of Flowing Systems with Internal Microstructure, first ed., Oxford University Press, Oxford, 1994] and by the Matrix Model [J. Rheol. 38 (1994) 769]. Minor differences with published results obtained by the Generalized Bracket are found in the equations describing flow coupled to heat and mass transfer in polymer solutions. The new method is applied to entangled polymer solutions and melts in the general case where the rate of generation of entanglements depends nonlinearly on the rate of strain. A link is drawn between the mesoscopic transport equations of entanglements and conformation and the microscopic equation describing the configurational distribution of polymer segment stretch and orientation. Constraints are derived on the generation terms in the transport equations of entanglements and conformation, and the formula for the elastic stress is generalized to account for reversible formation and destruction of entanglements. A simplified version of the transport equation of conformation is presented which includes many previously published constitutive models, separates flow-induced polymer stretching and orientation, yet is simple enough to be useful for developing large-scale computer codes for modeling coupled fluid flow and transport phenomena in two- and three-dimensional domains with complex shapes and free surfaces.
\end{abstract}

(C) 2004 Elsevier B.V. All rights reserved.

Keywords: Conformation tensor; Entanglements; Constitutive theory; Constitutive equations; Non-equilibrium thermodynamics; Generalized Bracket; Matrix Model; GENERIC

\section{Introduction}

Many process liquids used in the chemical, food, biomedical, coating, and polymer processing industries, are microstructured. Such liquids include polymer solutions and

\footnotetext{
* Corresponding author. Tel.: +1 713348 5830; fax: +1 7133485478.

E-mail address: mp@rice.edu (M. Pasquali).

${ }^{1}$ The main part of this research was conducted at the Department of Chemical Engineering and Materials Science of the University of Minnesota.
}

melts, liquid crystals, colloidal suspensions, emulsions, and many others. These liquids are not Newtonian; that is, they do not obey a simple linear relationship between stress and rate of strain. The flow behavior of these complex liquids can vary enormously [4-6]. However, liquids with like microstructure behave similarly in simple rheometric flows, and there is evidence that this similarity may carry over to complex process flows. Specifically, the dominant microstructural features of a linear polymer melt are the length and the stiffness of the polymer chains [7-12], which in turn control their degree of entanglement, i.e., the constraints 
that each chain poses to other chains' motion. The same properties are important in polymer solutions, in addition to concentration and to solvent quality, which may depend on temperature $[5,7,8,13]$. As another example, the behavior of emulsions is controlled by the volume fraction of the internal phase, the ratio of the viscosity of the internal and continuous phases, the interfacial tension between the two phases, and the type of interaction forces between droplets (attractive or repulsive). Of course, in polymeric emulsions the microstructural features of polymer solutions are also important.

Processing flows almost always include a combination of shear and extensional kinematics - the exception being fully developed flows in straight pipes and rectilinear channels. Extensional flow kinematics are important in flows where the thickness of a liquid sheet or filament changes, as in calendering, film blowing, and fiber spinning, and in regions of flows where a liquid film splits, as in forward roll coating, or accelerates and thins, as in slot, slide, and curtain coating.

Complex liquids behave differently in shear and extensional flows $[4,5]$. The length, stiffness, and degree of branching of polymer molecules strongly affect the shear and elongational response of polymer solutions and melts [5]. Polymeric liquids and other microstructured materials behave differently in shear and extensional flows because in extensional flows the liquid rotates locally with the rate of strain; therefore, the straining is persistent, i.e., it is always directed along the same direction from the material's perspective, whereas in shear flows, the material and the principal directions of the rate of strain rotate at different angular velocities [14-16].

Fast complex flows of polymer solutions have not yet been modeled well enough to permit accurate design of process equipment without first adjusting the model to match nearly the same flow. The main reason is the lack of theory that effectively accounts for the relevant non-equilibrium microstructural changes at time scales comparable with those of the process. The need for a sound theory is even greater when the typical rates of deformation achieved in the process flow exceed the ranges of state-of-the-art rheometers by an order of magnitude or more and the process flow kinematics depart substantially from the simple shear achievable in viscometric flows, as often occurs in these flows (e.g., coating, fiber spinning). Then theory is needed to project the information acquired in simple experiments to the complex reality of the process. The need for robust theories is even more pressing when tackling more complex transport phenomena, like coupled flow and heat transfer [17-22] or mass transfer [23-30]; experimental evidence on such processes is still limited [31-36].

This article is organized as follows. Section 2 compares and contrasts microscopic and mesoscopic approaches for modeling flows of polymer solutions and melts. Section 3 briefly recalls important theories and results for developing mesoscopic models of flowing liquids-namely, the continuum thermodynamic theory of Leonov, the Generalized
Bracket approach of Grmela and Beris and Edwards, the Matrix Model of Jongschaap, and the GENERIC framework of Grmela and Öttinger. In Section 4, general principles are introduced for developing mesoscopic thermodynamic theories with microstructural variables; such principles are applied to the specific case of polymeric liquids in Section 5, and simplified equation sets are presented in Section 6. Section 7 discusses the connection between microscopic and mesoscopic theories and derives expressions for the coupling between macroscopic flow and polymer conformation and entanglements; Section 8 derives the constraints on such coupling terms. Finally, Section 9 presents our conclusions and perspective on the usefulness of thermodynamically-consistent mesoscopic models.

\section{Coarse-grained and fine-grained theories}

The theories developed to describe the behavior of the microstructure of a material (liquid or solid) fall into two broad categories, loosely termed coarse-grained and fine-grained theories. The former are sometimes called mesoscopic, the latter microscopic. The two approaches differ mainly in the level of detail used to account for the material's microstructure.

The coarse-grained theories introduce field variables that are expectation values or "local average values" of microstructural features, like the average stretch and orientation of the end-to-end connectors of polymer molecules in a dilute polymer solution [12], or the orientation of the director of the nematic phase in a nematic liquid crystal [6,41]. Equations of change are then needed to describe how expectations of the microstructural features evolve in time and space, and how they interact with other mechanical and thermodynamic variables like velocity and temperature. The main advantage of the mesoscopic approach is economy: only a few field variables and differential equations are added to the usual mass, momentum, and internal energy conservation equations. The computational cost of solving flow and transport problems with this approach is therefore moderate and quite comparable to the cost of solving flow and transport problems with the classical constitutive equations that can be rationalized with linear irreversible local-equilibrium thermodynamics $[42,43]$, namely Newton's law of viscosity, Fourier's law of heat conduction, and Fick's law of mass diffusion. The main disadvantage of the mesoscopic approach is that there is no general way to formulate equations of change of microstructure. Several theories have been developed to do this, and those most relevant to our approach are briefly discussed below. However, these theories (including the one introduced in this article) provide no more than the general structure of the equations of change and a set of relationships and inequalities that restrict the functions that appear in them. Constitutive assumptions are still required to specify completely the behavior of a material. 
The fine-grained, or microscopic, approach represents the microstructural features of a material by means of a large number of micromechanical elements obeying stochastic differential equations or, equivalently, by the distribution in phase space of the state variables that describe a micromechanical element $[12,44]$. The equations of change of the microstructural element arise from a balance of momentum on the elementary mechanical components of the model-e.g., the beads of a chain of beads and springs or rods. In dilute solutions, the momentum balance usually neglects the inertia of the beads and includes the drag exerted by the solvent on the beads, the elastic or constraining intramolecular forces exerted by the springs or by the rods, and the Brownian forces representing the momentum exchanged between the polymer chains and the low molecular weight solvent molecules during collisions induced by their rapidly fluctuating velocities. Sometimes external fields are also considered [12]. In concentrated solutions and melts, the interaction of microstructural elements with each other is described through so-called "mean-field approximations" $[12,44]$ rather than with explicit interaction forces.

The main advantage of the microscopic approach is that it requires fewer assumptions about the forces acting on a micromechanical element of microstructure. Another important advantage of this approach is its potential [45-48] for representing molecular individualism observed in experiments with DNA solutions $[49,50]$. This behavior of DNA molecules is poorly approximated by the local average variables approach [45,51].

Microscopic models for the evolution of polymer microstructure can be coupled to macroscopic transport equations of mass and momentum to yield micro-macro models [52]. The main disadvantage of such a detailed accounting of microstructure is its computational cost, which is partly due to the lack of direct (rather than iterative time-stepping) algorithms for solving the coupled equations of macroscopic transport and microstructure evolution at once. In the simplest case, simulations with the most basic stochastic variables employ dumbbells, which require solving three scalar partial partial differential equations for each dumbbell field (or six differential equations for each trumbbell, etc.). The cost of introducing such a field of dumbbell configurations-e.g., by the Brownian Configuration Fields method of [53] - is roughly equivalent to the cost of introducing the conformation tensor or the elastic stress as an additional field variable (three or four scalar partial differential equations in two-dimensional flows, six scalar partial differential equations in three-dimensional flows). To obtain reliable statistics, stochastic methods must introduce and track approximately 1000 duplicate copies [53] of the dumbbell configuration field, which shows that the computational cost of stochastic methods is approximately two to three orders of magnitude larger than the cost of coarse-grained methods if the same solution algorithm is used. (Variance reduction [54] and other new approaches $[55,56]$ somewhat reduce this cost.) However, the available algorithms for solving flow problems with microstructural models have to rely on segregated sequential Picard (or Newton-Picard) iterations or on time-stepping to achieve steady-state solutions $[44,53,57-59]$. Such segregated algorithms converge slowly (if they converge at all), particularly when the equations are tightly coupled, as is the case when the elastic stresses induced by the non-equilibrium microstructure are large-e.g., in fast flows of polymer solutions and melts (see [60] for details).

Computations with micro-macro models are very expensive in two-dimensional flows and prohibitively so in three-dimensional flows with current algorithms and supercomputers. In certain problems with small-scale free surface flows dominated by capillarity (e.g., coating flows of polymer solutions), where the motion of the free surfaces is tightly coupled to the momentum equation, segregated methods are not nearly as robust as Newton's Method with initialization by continuation [61-63]. Moreover, process flow modeling aims at identifying the regions in the space of operating parameters where steady, stable flow is possible. The need to explore wide ranges of parameter space and the current high cost of computing a single flow state makes impracticable modeling complex flow processes with fine-grained models. However, the fine-grained (or micro-macro) approach may soon become viable, as more efficient computational algorithms are developed and faster massively parallel supercomputers become available, particularly because stochastic simulations are likely to take advantage of distributed memory parallel computers.

\section{Comparison of mesoscopic thermodynamic theories}

Several coarse-grained theories exist that describe the flowing microstructure of a polymeric liquid. These theories extend classical continuum thermodynamics based on the hypothesis of local equilibrium to include microstructural variables in the set of thermodynamic variables. The theories most relevant here are the thermodynamic theory of Leonov $[37,64,65]$, the Generalized Bracket formalism of Grmela [38,66], Grmela and Carreau [1], and Beris and Edwards [2,67,68], the Matrix Model of [3], and GENERIC $[40,69]$ (the GENERIC framework can handle the Boltzmann equation and does not require the local equilibrium assumption). Each is briefly described in this section. Other extensions of classical continuum thermodynamics with applications to polymeric liquids are discussed by Maugin and Drouot [70], Stickforth [71], Jou et al. [72], Maugin and Muschik [73,74], Drouot and Maugin [75], Muschik et al. [76], and Liu [77].

\subsection{The approach of Leonov}

Leonov's first work $[37,64]$ on using internal state variables to describe the rheological behavior of polymeric liquids originated from earlier theories of plastic flow of 
elasto-plastic materials [78]. Leonov's internal variable was originally the elastic Finger tensor $\boldsymbol{C}_{\mathrm{e}} \equiv \boldsymbol{F}_{\mathrm{e}} \cdot \boldsymbol{F}_{\mathrm{e}}^{\mathrm{T}}$, which is related to the elastic, or recoverable, part $\boldsymbol{F}_{\mathrm{e}}$ of the deformation gradient. The evolution equation for $\boldsymbol{C}_{\mathrm{e}}$ was coupled to the macroscopic deformation through an upper-convected derivative, similarly to the evolution equation of the Finger tensor $\boldsymbol{B}$ :

$\stackrel{\nabla}{\boldsymbol{B}} \equiv \dot{\boldsymbol{B}}-\nabla \boldsymbol{v}^{\mathrm{T}} \cdot \boldsymbol{B}-\boldsymbol{B} \cdot \nabla \boldsymbol{v}=0$.

Here the overdot denotes the material time derivative. Eq. (1) is a purely kinematic relationship that follows from the definition of the Finger tensor $\boldsymbol{B} \equiv \boldsymbol{F} \cdot \boldsymbol{F}^{\mathrm{T}}$ in terms of the deformation gradient $\boldsymbol{F}$, and from the material time derivative of the deformation gradient [79]:

$\dot{\boldsymbol{F}}=\nabla \boldsymbol{v}^{\mathrm{T}} \cdot \boldsymbol{F}$.

or, in component form,

$\dot{F}_{i j}=\frac{\partial \dot{x}_{i}}{\partial x_{j}^{0}}=\frac{\partial v_{i}}{\partial x_{k}} \frac{\partial \dot{x}_{k}}{\partial x_{j}^{0}}$

where $\partial x_{j}^{0}$ denotes the position of a material point in the reference (undeformed) configuration of the body and $x_{k}$ is the position of the same point in the deformed configuration; summation over repeated indices is implied.

Leonov [37] built the relaxational part of the evolution equation of $\boldsymbol{C}_{\mathrm{e}}$ from a non-equilibrium dissipative potential, and constrained the relaxational term to maintain the condition $\operatorname{det} \boldsymbol{C}_{\mathrm{e}}=1$, which insures that the elastic part of the deformation is isochoric. The elastic stress $\sigma$ followed from the derivative of the specific Helmholtz free energy $a$ with respect to the recoverable strain, i.e., the elastic strain:

$\boldsymbol{\sigma}=2 \rho \boldsymbol{C}_{\mathrm{e}} \cdot \frac{\partial a}{\partial \boldsymbol{C}_{\mathrm{e}}}$.

(See Appendix A for the expression of the derivative of a scalar with respect to a tensor.) Conversely, the only admissible Helmholtz free energy must be the isothermal integral of this relation. In theories of elastic materials, internal, Helmholtz, and other free energies are ultimately based on "reversible work", i.e., the path-independent integral of the product of the elastic stress multiplied by the incremental elastic strain; Eq. (4) generalizes this concept to include integrals of elastic-like internal variables.

More recently Leonov [65] and Leonov and Prokunin [80] extended this theory to include most of the models that have been developed from molecular theories by generalizing the elastic strain tensor to a configuration tensor $\boldsymbol{M}$ (Leonov used the symbol $\boldsymbol{C}$ ) representing the second moment of the end-to-end connector of the polymer coils in a polymer solution or melt (or the connector between successive entanglements). In doing so, Leonov relaxed the constraint det $\boldsymbol{M}=1$ in order to include many rate-type constitutive equations in his framework. The stress-configuration relationship of Eq. (4) stayed unchanged. The later theory of [65] and [80] is briefly outlined below.
Leonov's premises are that the Helmholtz free energy per unit mass $a$ depends on the configuration tensor $\boldsymbol{M}$ and on temperature $T$, i.e., $a=a(\boldsymbol{M}, T)$, and that the elastic stress $\sigma$ depends on the derivative of the free energy with respect to the configuration tensor in the same way as it depends on elastic strain,

$\sigma=2 \rho \boldsymbol{M} \cdot \boldsymbol{S}$,

where $\boldsymbol{S} \equiv \partial a / \partial \boldsymbol{M}$. Leonov then assumes that the appropriate form of the evolution equation of $\boldsymbol{M}$ has as the time derivative of configuration a corotational, or Jaumann, derivative,

$$
\begin{aligned}
\stackrel{\circ}{\boldsymbol{M}} & \equiv \frac{\partial \boldsymbol{M}}{\partial t}+\boldsymbol{v} \cdot \nabla \boldsymbol{M}-\boldsymbol{W}^{\mathrm{T}} \cdot \boldsymbol{M}-\boldsymbol{M} \cdot \boldsymbol{W} \\
& =\boldsymbol{D}_{\mathrm{e}} \cdot \boldsymbol{M}+\boldsymbol{M} \cdot \boldsymbol{D}_{\mathrm{e}},
\end{aligned}
$$

where

$$
\boldsymbol{W} \equiv \frac{1}{2}\left(\nabla \boldsymbol{v}-\nabla \boldsymbol{v}^{\mathrm{T}}\right)
$$

is the vorticity dyadic, ${ }^{2}$ and $\boldsymbol{D}_{\mathrm{e}}$ is called the elastic rate of strain; thus, he tacitly assumes that the configuration tensor translates with the liquid's velocity and rotates with the liquid's angular velocity - half the vorticity. Eq. (6), in fact, defines the elastic rate of strain $\boldsymbol{D}_{\mathrm{e}}$; its complement is the rate of irreversible strain $D_{\mathrm{p}}$ :

$\boldsymbol{D}_{\mathrm{e}}+\boldsymbol{D}_{\mathrm{p}} \equiv \boldsymbol{D} \equiv \frac{1}{2}\left(\nabla \boldsymbol{v}+\nabla \boldsymbol{v}^{\mathrm{T}}\right)$.

A relationship between the irreversible rate of strain $\boldsymbol{D}_{\mathrm{p}}$ and the other variables, i.e., a constitutive equation, must be specified to close the equation set.

Leonov notes that, under his premises, the rate of entropy generation per unit volume $s_{g}$ can be written as

$s_{\mathrm{g}}=-\frac{1}{T} \boldsymbol{q} \cdot \nabla T+\frac{1}{T} \boldsymbol{\sigma}: \boldsymbol{D}_{\mathrm{p}}$.

In linear irreversible thermodynamics, the rate of entropy generation is usually written as the product of what are called thermodynamic forces $X_{k}$ and thermodynamic fluxes $J_{k}$, as

$s_{\mathrm{g}}=\sum_{k} X_{k} J_{k}$

and the thermodynamic fluxes are in turn related to the thermodynamic forces through the matrix of phenomenological coefficients $\mathcal{L}$, as $J_{k}=\sum_{i} L_{k i} J_{i}$ [43]. Adopting this formalism, Leonov identifies $\sigma$ with a thermodynamic force and $\boldsymbol{D}_{\mathrm{p}}$ with the corresponding thermodynamic flux, and writes their relationship as $D_{\mathrm{p}}=N: \sigma$, where $N$ is a fourth-rank tensor that could depend on the configuration tensor and on the temperature. $N$ should be positive definite to guarantee that the rate of entropy generation in Eq. (8) be non-negative.

\footnotetext{
${ }^{2}$ In this article the term dyadic is used in the original definition of Gibbs [81].
} 
Because $\stackrel{\circ}{\boldsymbol{M}}-\boldsymbol{D} \cdot \boldsymbol{M}-\boldsymbol{M} \cdot \boldsymbol{D}=\stackrel{\nabla}{\boldsymbol{M}}$, the complete set of equations of Leonov's theory is

$\stackrel{\nabla}{M}+2 M \cdot N(M, T): \sigma=0$

$\sigma=2 \rho \boldsymbol{M} \cdot \boldsymbol{S}$

together with the constitutive equations of $N(M, T)$ and $a(\boldsymbol{M}, T)$.

The coupling between the configuration tensor and the velocity gradient in Eqs. (11) and (12) is expressed by means of an upper-convected derivative, which carries the implicit assumption that the polymer molecules are deforming affinely [5], i.e., the molecular strain equals the local macroscopic strain; therefore, Eqs. (11) and (12) do not include constitutive equations that allow slip between the polymer molecules and the surrounding liquid, such as the Johnson-Segalman equation [82], the Phan-Thien and Tanner equation [83,84], and the Larson equation [85].

Leonov generalized Eqs. (11) and (12) by introducing the concept of a non-equilibrium stress tensor $\sigma_{\text {ne }} \equiv \sigma / \xi$ and a non-equilibrium irreversible rate of strain in contrast to the equilibrium irreversible rate of strain $\boldsymbol{D}_{\mathrm{ne}} \equiv \xi \boldsymbol{D}_{\mathrm{p}}$, where $\xi$ is a numerical parameter, $-1 \leq \xi \leq 1$. The set of equations that includes non-equilibrium stress and irreversible rate of strain is then

$\stackrel{\circ}{\boldsymbol{M}}-\xi(\boldsymbol{D} \cdot \boldsymbol{M}+\boldsymbol{M} \cdot \boldsymbol{D})+\boldsymbol{E}(\boldsymbol{M}, T)=0$

$\sigma=\frac{2 \rho}{\xi} \boldsymbol{M} \cdot \boldsymbol{S}$,

or, in terms of upper convected derivative,

$\stackrel{\nabla}{\boldsymbol{M}}+(1-\xi)(\boldsymbol{D} \cdot \boldsymbol{M}+\boldsymbol{M} \cdot \boldsymbol{D})+\boldsymbol{E}(\boldsymbol{M}, T)=0$

$\boldsymbol{\sigma}=\frac{2 \rho}{\xi} \boldsymbol{M} \cdot \boldsymbol{S}$

where $\boldsymbol{E}(\boldsymbol{M}, T)$ is an isotropic function of its arguments, called the relaxational part of the evolution equation of $\boldsymbol{M}$. Writing the relaxational part of Eq. (15) as $\boldsymbol{E}(\boldsymbol{M}, T)$ is equivalent to writing it as $N: \sigma$ because both $N$ and $\sigma$ depend on $\boldsymbol{M}$ and $T$ only.

Eqs. (15) and (16) are general enough to include most known rate-type constitutive equations [80]. Leonov [65] rejects equations of change of $\boldsymbol{M}$ where the deformationdependent part differs from the standard lower $(\xi=-1)$ or upper $(\xi=1)$ convected derivatives (see also [86]); mixed derivatives are rejected because these give rise to Hadamard unstable equations of motion, i.e., equations of motion whose solution does not depend continuously on the initial and boundary conditions [[87], p. 227]. Of course, Hadamard instabilities are suppressed if the stress tensor has a (even minute) viscous component-i.e., a component linearly proportional to the rate of strain.

The main merits of Leonov's approach are conciseness and economy. However, this approach carries several limitations. Leonov postulates independently (1) that the
(Helmholtz) free energy depends on the the configuration tensor; (2) the relationship between the rate of change of the configuration tensor and the rate of strain tensor; and (3) the the relationship between elastic stress and configuration tensor. The analysis in Section 5.3 below shows that only the first two postulates can be made independently, whereas the relationship between elastic stress and configuration tensor follows from the requirement that the local rate of entropy production must be non-negative-i.e., the local form of the second law of thermodynamics.

Moreover, Leonov's theory offers no indication on how to extend the relationship between elastic stress and conformation to the case of entangled polymeric liquids when an explicit entanglement density variable is introduced. Also, Leonov offers no provisions on how to include effects of molecular conformation-induced migration in the evolution equation of the configuration tensor. Clearly, the theory is rooted in elasticity and plasticity, and it can be related to macroscopic transport theories and to molecular theories built on statistical mechanics only with ad hoc adjustments (e.g., those of Eqs. (15) and (16)).

\subsection{The Generalized Bracket approach of Grmela, Beris, and Edwards}

In analytical mechanics of idealized, conservative systems of particles or bodies, the time evolution of a closed, isolated system is sometimes expressed in terms of Poisson brackets, which are useful because they are invariant under canonical transformations [88], i.e., transformations that preserve the canonical form of Hamilton's equations (Eqs. (17) and (18)). In analytical mechanics, Newton's equations of motion for a closed system subject to conservative forces are rewritten in terms of generalized coordinates, positions $q_{i}$ and momenta $p_{i}$, as

$\dot{q}_{i}=\frac{\partial H}{\partial p_{i}}, \quad i=1, N$

$\dot{p}_{i}=-\frac{\partial H}{\partial q_{i}}, \quad i=1, N$,

where $H\left(q_{1}, q_{2}, \ldots, q_{N}, p_{1}, p_{2}, \ldots, p_{N}\right)$ is the Hamiltonian of the system, i.e., the total mechanical energy, and $N$ is the number of degrees of freedom of the system. If there are two generic functions, $u, v$, that depend on only the generalized coordinates $q_{i}, p_{i}$, then the Poisson bracket $\{u, v\}$ can be defined usefully as

$\{u, v\} \equiv \sum_{i=1}^{N}\left(\frac{\partial u}{\partial q_{i}} \frac{\partial v}{\partial p_{i}}-\frac{\partial v}{\partial q_{i}} \frac{\partial u}{\partial p_{i}}\right)$.

Because of this definition, the equations of change of any function of the generalized coordinates $u\left(q_{i}, p_{i}\right)$ can be written in the form

$\frac{\mathrm{d} u}{\mathrm{~d} t}=\{u, H\}$. 
Eq. (20) can be derived by evaluating the time rate of change of $u$ by means of implicit differentiation and recalling Eqs. (17) and (18):

$$
\begin{aligned}
& \frac{\mathrm{d}}{\mathrm{d} t} u\left(q_{i}, p_{i}\right)=\sum_{i=1}^{N}\left(\frac{\partial u}{\partial q_{i}} \frac{\mathrm{d} q_{i}}{\mathrm{~d} t}+\frac{\partial u}{\partial p_{i}} \frac{\mathrm{d} p_{i}}{\mathrm{~d} t}\right) \\
& \frac{\mathrm{d}}{\mathrm{d} t} u\left(q_{i}, p_{i}\right)=\sum_{i=1}^{N}\left(\frac{\partial u}{\partial q_{i}} \frac{\partial H}{\partial p_{i}}-\frac{\partial u}{\partial p_{i}} \frac{\partial H}{\partial q_{i}}\right) \\
& \frac{\mathrm{d}}{\mathrm{d} t} u\left(q_{i}, p_{i}\right) \equiv\{u, H\} .
\end{aligned}
$$

The Poisson bracket is a bilinear and antisymmetric operator, i.e.,

$$
\begin{aligned}
& \{\alpha u, v\}=\alpha\{u, v\} \\
& \{u+v, w\}=\{u, w\}+\{v, w\} \\
& \{u, v\}=-\{v, u\}
\end{aligned}
$$

therefore $\{u, u\} \equiv 0$. This property guarantees the conservation of total energy of a system whose evolution is described by the Poisson bracket:

$$
\frac{\mathrm{d}}{\mathrm{d} t} H=\{H, H\}=0 .
$$

Other properties of the Poisson bracket are discussed by [2].

The Poisson bracket can be extended to conservative continuous systems, for example, the ideal fluid and the non-linearly elastic solid ([67] and Ch. 5 of [2]). Kaufman [89], Morrison [90], and Grmela [66] published the first attempts to include dissipation in systems described by bracket formalisms by introducing a dissipation bracket. Grmela and Carreau [1], Grmela [38,91], and Beris and Edwards $[2,67,68]$ used the bracket formalism to formulate the equations of change of polymeric liquids in a unified framework based on introducing the conformation tensor, an expectation value or continuum variable that represents local average values of stretch and orientation of the polymer coils. The basic premise is that the dynamics of an isolated system can be described by the equation [[2], Ch. 7]

$\frac{\mathrm{d} F}{\mathrm{~d} t}=\{[F, H]\} \equiv F, H+[F, H]$

where $\{[\cdot, \cdot]\}$ is called the Generalized Bracket, $\{\cdot, \cdot\}$ is the Poisson bracket, $[\cdot, \cdot]$ is the dissipation bracket, $H$ is the Hamiltonian of the system, and $F$ is any function of the state variables. Beris and Edwards motivate this premise by pointing out that Eq. (28) includes as limiting cases Eqs. (17) and (18) of Hamiltonian dynamics and Eq. (10) of irreversible thermodynamics. Under this premise, the equations of change of the total mass $M$, energy $H$, and entropy $S$ of the system are

$$
\frac{\mathrm{d} M}{\mathrm{~d} t}=\{[M, H]\} \equiv\{M, H\}+[M, H]
$$

$$
\begin{aligned}
& \frac{\mathrm{d} H}{\mathrm{~d} t}=\{[H, H]\} \equiv\{H, H\}+[H, H]=[H, H] \\
& \frac{\mathrm{d} S}{\mathrm{~d} t}=\{[S, H]\} \equiv\{S, H\}+[S, H] .
\end{aligned}
$$

The antisymmetric property of the Poisson bracket Eq. (25) guarantees that $\{H, H\}=0$ in Eq. (30). The system's mass and energy must be conserved because the system is isolated,

$$
\begin{aligned}
\frac{\mathrm{d} M}{\mathrm{~d} t} & =0 \\
\frac{\mathrm{d} H}{\mathrm{~d} t} & =0,
\end{aligned}
$$

whereas the entropy of the system may rise in time because of irreversible processes,

$\frac{\mathrm{d} S}{\mathrm{~d} t} \geq 0$.

In a continuum system, the Poisson bracket represents the rate of change of any quantity due to reversible processes [2]. The total mass of an isolated system does not change because of reversible processes; thus $\{M, H\}=0 .\{S, H\}$ represents the reversible rate of change of entropy; therefore, the Poisson bracket should be built to satisfy $\{S, H\}=0$, because the entropy of an isolated system cannot change due to reversible processes.

These properties of the Poisson bracket, together with the conservation laws of mass Eq. (29), total energy Eq. (30), and entropy Eq. (31) for a closed, isolated system pose conditions on the dissipation bracket:

$$
\begin{array}{ll}
{[H, H]=0} & \text { conservation of energy } \\
{[M, H]=0} & \text { conservation of mass } \\
{[S, H] \geq 0} & \text { second law of thermodynamics. }
\end{array}
$$

Moreover, since the time rate of change of $F$ is linear in $F$, the dissipative bracket must be linear in $F$ too. From the requirements in Eqs. (35)-(37), Beris and Edwards build the most general dissipation bracket admissible for an isolated, closed continuum ([2], Eq. (7.1-19)). Beris and Edwards point out the similarity of postulate (28) to the equations proposed by Prigogine et al. [92] in their unified formulation of dynamics and thermodynamics.

Beris and Edwards ([2], Ch. 8) put forward a unified account of the dynamics of incompressible, isothermal viscoelastic liquids developed by using generalized brackets. They state that the only variables needed to describe the state of the system are the momentum density $\boldsymbol{p} \equiv \rho \boldsymbol{v}$, and the conformation density $\hat{\boldsymbol{M}} \equiv \rho c_{\mathrm{p}} \boldsymbol{M}$, where $\rho$ is the liquid's density, $c_{\mathrm{p}}$ is the number of polymer molecules per unit mass, $\boldsymbol{v}$ is the liquid's velocity, and $\boldsymbol{M} \equiv\langle\boldsymbol{r} \boldsymbol{r}\rangle$ is the conformation tensor (Beris and Edwards used the symbol $\boldsymbol{C}$ for the conformation tensor). The vector $\boldsymbol{r}$ is the end-to-end connector of a polymer coil and the symbol $\langle\cdot\rangle$ indicates average over all possible realizations in phase space. 
The Poisson bracket of this system is taken to be identical to that of a nonlinearly elastic material (non-dissipative), because of the similarity between the conformation tensor and the Finger strain tensor $\boldsymbol{B} \equiv \boldsymbol{F} \cdot \boldsymbol{F}^{\mathrm{T}}$, where $\boldsymbol{F}$ is the deformation gradient. In a crosslinked rubber, $\boldsymbol{M}=N \ell^{2} \boldsymbol{B} / 3$, where $N$ is the number of statistical segments in a chain between two crosslinks and $\ell$ is the length of a statistical segment.

The dissipation bracket $[F, G]$ is built as a bilinear form to exclude nonlinear transport effects [2]. $[F, G]$ is written in terms of the independent variables as ([2], Eq. (8.1-5))

$$
\begin{aligned}
{[F, G]=} & -\int_{\Omega} \frac{\partial f}{\partial \boldsymbol{M}}: \boldsymbol{\Lambda}: \frac{\partial g}{\partial \boldsymbol{M}} d \Omega \\
& -\int_{\Omega} \nabla \frac{\partial f}{\partial \boldsymbol{M}} \bullet \boldsymbol{B} \bullet \nabla \frac{\partial g}{\partial \boldsymbol{M}} d \Omega \\
& -\int_{\Omega} \nabla \frac{\partial f}{\partial \boldsymbol{p}}: \boldsymbol{Q}: \nabla \frac{\partial g}{\partial \boldsymbol{p}} d \Omega \\
& -\int_{\Omega}\left(\nabla \frac{\partial f}{\partial \boldsymbol{p}}: \boldsymbol{L}: \frac{\partial g}{\partial \boldsymbol{M}}-\nabla \frac{\partial g}{\partial \boldsymbol{p}}: \boldsymbol{L}: \frac{\partial f}{\partial \boldsymbol{M}}\right) d \Omega
\end{aligned}
$$

where the symbol $(\bullet)$ is defined by $\boldsymbol{a b c} \bullet \boldsymbol{d e f} \equiv \boldsymbol{c b a}: \boldsymbol{d e} \boldsymbol{f} \equiv$ $(\boldsymbol{a} \cdot \boldsymbol{d})(\boldsymbol{b} \cdot \boldsymbol{e})(\boldsymbol{c} \cdot \boldsymbol{f})$. In Eq. (38) the Volterra functional derivatives of the extensive variables $F$ and $G$ used by Beris and Edwards [2] have been replaced by partial derivatives of their densities $f$ and $g$ with respect to the state variables $\boldsymbol{p}$, and $\boldsymbol{M}$ because hereafter the functions $F \equiv \int_{\Omega} f \mathrm{~d} \Omega$ and $G \equiv \int_{\Omega} g \mathrm{~d} \Omega$ are supposed to depend on the state variables but not on their spatial gradients. The first term in Eq. (38) represents the dissipative effects due to molecular relaxation; the second term characterizes dissipation due to molecular diffusion; and the third term expresses the effects of viscous dissipation. The fourth term of Eq. (38) is not dissipative and represents interactions between the conformation tensor and the velocity gradient other than those embedded in the Poisson bracket borrowed from the elastic solid. The coupling between elastic strain and velocity gradient in the Poisson bracket of a nonlinearly elastic solid is equivalent to the assumption of affine deformation of the microstructure because elastic solids deform affinely by definition; therefore, the fourth-rank tensor $\boldsymbol{L}$ characterizes non-affine deformation of the microstructure. Beris and Edwards [2] comment on the meaning of this coupling and point out that non-affine deformation cannot be included in the Poisson bracket because it violates the Jacobi identity, even though it gives rise to an antisymmetric, non-dissipative term in the dissipation bracket. This term was not included in the first articles of Beris and Edwards and was introduced later [93]. The possibility of non-affine deformation of microstructure and its connections with elastic stress and the momentum equations are discussed in Section 7.2.

In the basic theory, Beris and Edwards assume that the liquid is incompressible and neglect diffusion, as has been customary in developing theories of flow of polymer solutions and melts, and set the sixth-rank tensor $\boldsymbol{B} \equiv \mathbf{0}$; diffusion is considered in Ref. [2] (Ch. 9). The final form of the transport equations they obtain is ([2], Eq. (8.1-7))

$$
\begin{aligned}
& \frac{\partial \rho}{\partial t}=\nabla \cdot \boldsymbol{v} \rho \\
& \rho \frac{\partial \boldsymbol{v}}{\partial t}=-\rho \boldsymbol{v} \cdot \nabla \boldsymbol{v}-\nabla p+\nabla \cdot \boldsymbol{\sigma} \\
& \underbrace{\frac{\partial \boldsymbol{M}}{\partial t}}_{\text {convection }}=-\underbrace{\boldsymbol{v} \cdot \nabla \boldsymbol{M}}_{\text {affine deformation }} \\
& \text { rate of change } \\
& \text { of conformation } \\
& -\underbrace{\boldsymbol{\Lambda}: \boldsymbol{S}}_{\text {relaxation }}+\underbrace{\boldsymbol{L}: \nabla \boldsymbol{v}}_{\text {non-affine deformation }} \\
& \underbrace{\sigma}_{\text {extra stress }}=\underbrace{\boldsymbol{Q}: \nabla v}_{\text {viscous stress }}+\underbrace{2 \boldsymbol{M} \cdot S}_{\begin{array}{c}
\text { elastic stress induced } \\
\text { by affine deformation }
\end{array}} \\
& +\underbrace{2 \boldsymbol{L}: S}_{\text {elastic stress induced by non-affine deformation }},
\end{aligned}
$$

where $\boldsymbol{S} \equiv \partial a / \partial \boldsymbol{M}$ and $a(\boldsymbol{M})$ is the isothermal Helmholtz free energy per unit volume; therefore, $a(\boldsymbol{M})$ is the reversible work stored in a certain class of processes. The mechanical pressure $p$ is constitutively indeterminate because the liquid is incompressible.

Particular models of viscoelastic behavior are recovered from Eqs. (39)-(42) by specifying appropriate expressions of the free energy $a(\boldsymbol{M})$ and the phenomenological tensors $\boldsymbol{Q}, \boldsymbol{\Lambda}$, and $\boldsymbol{L}$. Moreover, the tensors $\boldsymbol{Q}, \boldsymbol{\Lambda}$ and the free energy must satisfy the conditions imposed by the second law of thermodynamics:

$S: \Lambda: S \geq 0$

$\nabla \boldsymbol{v}: \boldsymbol{Q}: \nabla \boldsymbol{v} \geq 0$.

Eq. (43) states that the free energy of the liquid must diminish during the spontaneous process of molecular relaxation, and Eq. (44) states that the rate of working of the viscous stress must contribute to the local rate of entropy production. The two inequalities must hold independently; therefore, molecular relaxation and viscous flow must generate entropy separately.

The Generalized Bracket formalism for dealing with flow and transport in viscoelastic media offers several advantages - and some disadvantages as well. In the bracket context, it is clear how to introduce more field variables into a model of a microstructured material, and how to describe transport in rather general situations ([2], Ch. 9). Moreover, the theory of flow and transport in polymeric liquids developed through the Generalized Bracket is closely connected to molecular theories based on micromechanical models, although GENERIC seems better suited to describe the connection between the fine-grained and coarse-grained levels of description [40,69,94,95].

The bracket approach is not free of drawbacks. In the authors' opinion, the mathematics used is so complex as to 


\section{Bracket Approach to Thermodynamics}

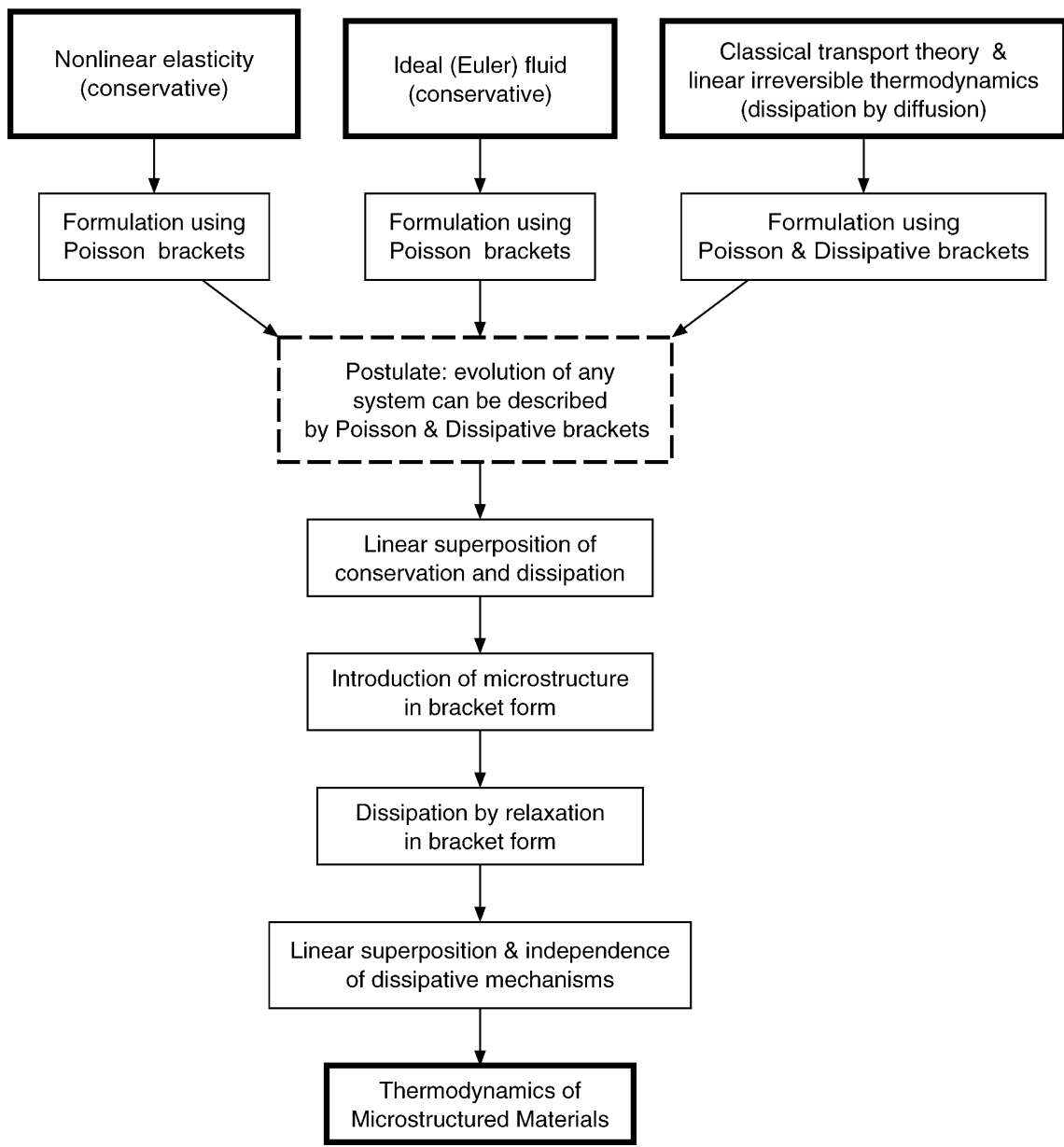

Fig. 1. Schematic development of the Generalized Bracket approach to the thermodynamics of microstructured materials. The Generalized Bracket approach has been very useful in developing theories of flow and transport in polymeric liquids [2]. The work of Grmela and Öttinger [40] and the analysis presented in Sections 4-7 show that equally powerful approaches are available that rely on simpler mathematics.

be sometimes opaque and the concepts are not introduced in the plainest and most intuitive way. The equations are neither used nor solved in the bracket form, and the bracket is used only to generate the partial differential equations of transport as diagrammed in Fig. 1.

Although the laws of conservation of mass and energy and the second law of thermodynamics are embedded in the bracket structure, the system's transport equations must be postulated because the bracket formalism-like any other thermodynamic formalism-does not provide explicit formulae for transport coefficients and generation terms. Building a specific form of the Poisson and dissipation brackets of a system requires making assumptions on how the microstructure of the material and the flow interact and affect each other; these assumptions enter the model through the constitutive equations of the generation (or relaxation) terms and the diffusive transport coefficients, as usually happens in thermodynamics. It seems therefore more natural to seek an extension of thermodynamics of irreversible processes rather than the bracket formalism to check existing models of microstructured materials and to develop new ones.

\subsection{The Matrix Model of Jongschaap}

Jongschaap [39] and Jongschaap et al. [3] used internal variables to develop a unified theory of isothermal flows of non-diffusing polymer solutions and melts. The internal variable of choice was the conformation tensor $\boldsymbol{M}$ (Jongschaap et al. used the symbol $\boldsymbol{S}$ ). In isothermal, incompressible flows, Gibbs' fundamental equation is $a=a(\boldsymbol{M})$, where $a$ is the Helmholtz free energy per unit volume of the liquid. The dissipation is

$T s_{\mathrm{g}}=\boldsymbol{T}: \nabla \boldsymbol{v}-\boldsymbol{S}: \dot{\boldsymbol{M}}$

where $\boldsymbol{T}$ is the stress dyadic, $\boldsymbol{S} \equiv \partial a / \partial \boldsymbol{M}$, and the overdot denotes material time derivative. Jongschaap et al. [3] (p. 775) introduce the principle of macroscopic time rever- 
sal to distinguish reversible and irreversible contributions to Eq. (45):

The value of the state variables in the Gibbs fundamental equation $[a=a(\boldsymbol{M})]$ and the dissipation $\left[T s_{\mathrm{g}}\right]$ defined by Eq. (45) remain unchanged under a reversal of the rates of change of the external rate variables.

Jongschaap et al. motivate this assumption by stating that

The dissipation $\left[T s_{\mathrm{g}}\right]$, being the result of rapid internal fluctuations of variables which are not directly coupled to the external variables, is expected to be invariant under a macroscopic time reversal.

According to Jongschaap's theory, the only external rate variable in Eq. (45) is the velocity gradient. The stress $\boldsymbol{T}$ and the rate of change of conformation $\dot{\boldsymbol{M}}$ are decomposed into reversible and irreversible parts according to whether or not their contribution to the dissipation changes sign when the velocity gradient is reversed:

$$
\begin{aligned}
& \boldsymbol{T}(\nabla \boldsymbol{v}, \boldsymbol{S}) \equiv \boldsymbol{T}^{\mathrm{r}}(\nabla \boldsymbol{v}, \boldsymbol{S})+\boldsymbol{T}^{\mathrm{i}}(\nabla \boldsymbol{v}, \boldsymbol{S}) \\
& \dot{\boldsymbol{M}}(\nabla \boldsymbol{v}, \boldsymbol{S}) \equiv \dot{\boldsymbol{M}}^{\mathrm{r}}(\nabla \boldsymbol{v}, \boldsymbol{S})+\dot{\boldsymbol{M}}^{\mathrm{i}}(\nabla \boldsymbol{v}, \boldsymbol{S}),
\end{aligned}
$$

with

$$
\begin{aligned}
& \boldsymbol{T}^{\mathrm{r}}(-\nabla \boldsymbol{v}, \boldsymbol{S}) \equiv \boldsymbol{T}^{\mathrm{r}}(\nabla \boldsymbol{v}, \boldsymbol{S}) \\
& \boldsymbol{T}^{\mathrm{i}}(-\nabla \boldsymbol{v}, \boldsymbol{S}) \equiv-\boldsymbol{T}^{\mathrm{i}}(\nabla \boldsymbol{v}, \boldsymbol{S}) \\
& \dot{\boldsymbol{M}}^{\mathrm{r}}(-\nabla \boldsymbol{v}, \boldsymbol{S}) \equiv-\dot{\boldsymbol{M}}^{\mathrm{r}}(\nabla \boldsymbol{v}, \boldsymbol{S}) \\
& \dot{\boldsymbol{M}}^{\mathrm{i}}(-\nabla \boldsymbol{v}, \boldsymbol{S}) \equiv \dot{\boldsymbol{M}}^{\mathrm{i}}(\nabla \boldsymbol{v}, \boldsymbol{S}) .
\end{aligned}
$$

Eq. (45) and the aforementioned principle of macroscopic time reversal give

$$
\begin{aligned}
& \boldsymbol{T}^{\mathrm{r}}: \nabla \boldsymbol{v}-\boldsymbol{S}: \dot{\boldsymbol{M}}^{\mathrm{r}}=0 \\
& T s_{\mathrm{g}}=\boldsymbol{T}^{\mathrm{i}}: \nabla \boldsymbol{v}-\boldsymbol{S}: \dot{\boldsymbol{M}}^{\mathrm{i}} \geq 0 .
\end{aligned}
$$

The reversible part of the rate of change of conformation is written as

$\dot{\boldsymbol{M}}^{\mathrm{r}} \equiv \tilde{\boldsymbol{\Lambda}}(\nabla \boldsymbol{v}, S): \nabla \boldsymbol{v}$

where $\tilde{\boldsymbol{\Lambda}}$ is a fourth-rank tensor, and $\tilde{\boldsymbol{\Lambda}}(-\nabla \boldsymbol{v}, \boldsymbol{S})=$ $\tilde{\boldsymbol{\Lambda}}(\nabla \boldsymbol{v}, \boldsymbol{S})$.

The reversible (elastic) part of the stress is thus

$\boldsymbol{T}^{\mathrm{r}}=\boldsymbol{S}: \tilde{\boldsymbol{\Lambda}}=\tilde{\boldsymbol{\Lambda}}^{\mathrm{T}}: \boldsymbol{S}$,

where $\tilde{\boldsymbol{\Lambda}}_{i j k l}^{\mathrm{T}}=\tilde{\boldsymbol{\Lambda}}_{k l i j}$. The irreversible part of the stress and the rate of change of conformation are

$\boldsymbol{T}^{\mathrm{i}} \equiv \boldsymbol{\eta}(\nabla \boldsymbol{v}, \boldsymbol{S}): \nabla \boldsymbol{v}$

$\dot{\boldsymbol{M}}^{\mathrm{i}} \equiv-\boldsymbol{\beta}(\nabla \boldsymbol{v}, \boldsymbol{S}): \boldsymbol{S}$,

where $\boldsymbol{\eta}$ and $\boldsymbol{\beta}$ are fourth-rank tensors. According to Jongschaap et al. [3], $\boldsymbol{\eta}$ and $\boldsymbol{\beta}$ must be positive semi-definite to guarantee that the entropy production rate is not negative.
More precisely, $\boldsymbol{\eta}$ and $\boldsymbol{\beta}$ must be positive semi-definite only if they are independent of $\nabla \boldsymbol{v}$ and $\boldsymbol{S}$ [60]. The tensor $\tilde{\boldsymbol{\Lambda}}$ can then be decomposed into a part that multiplies the rate of strain and a part that multiplies the vorticity:

$$
\dot{\boldsymbol{M}}^{\mathrm{r}} \equiv \tilde{\boldsymbol{\Lambda}}(\nabla \boldsymbol{v}, \boldsymbol{S}): \nabla \boldsymbol{v} \equiv \boldsymbol{\Lambda}^{0}: \boldsymbol{W}+\boldsymbol{\Lambda}^{1}: \boldsymbol{D} .
$$

Jongschaap et al. [3] argue that the entropy production rate should be independent of the choice of (rigid) frame of reference, which implies that

$\boldsymbol{\Lambda}_{i j k l}^{0}=\frac{1}{2}\left(M_{i l} \delta_{j k}+M_{j l} \delta_{i k}-M_{i k} \delta_{j l}-M_{i l} \delta_{j k}\right)$.

Jongschaap et al. [3] use isotropic representation theorems to write general formulae for the tensors $\boldsymbol{\Lambda}^{1}, \boldsymbol{\eta}$, and $\boldsymbol{\beta}$, and show that several models of viscoelastic behavior are included in their Matrix Model and can be recovered by specifying particular forms of the tensors $\boldsymbol{\Lambda}^{1}$ and $\boldsymbol{\beta}$ (e.g, upper-convected Maxwell; Leonov [37]; Johnson and Segalman [82]; Doi [96]; Giesekus [97]; Larson [85]; and finitely extensible nonlinearly elastic dumbbell [12]). Finally, Jongschaap et al. remark that because these two tensors are independent of each other, it is legitimate to choose the expression of $\boldsymbol{\Lambda}^{1}$ suggested by one molecular model, and the expression of $\boldsymbol{\beta}$ suggested by a different model, forming thus new hybrid models. Such a suggestion has very interesting ramifications for the development of modular computer codes for large-scale computational modeling of flow and transport in microstructured liquids (complex fluids) because it permits the separation of different transport phenomena and different parts of constitutive equations into small computer modules (objects) at the time of code-writing. Such objects can be assembled when the computer codes are compiled (once specific constitutive equations have been selected). Creating "hybrid" constitutive equations becomes then as easy as selecting objects (or subroutines) from a library, and new constitutive equations can be introduced with minimal effort—-see Pasquali [60] for details, Pasquali and Scriven [63] and Zevallos et al. [98] for the application to two-dimensional free surface flows, Xie and Pasquali $[99,100]$ for the extension to three-dimensional flows, and Guénette [101] for an early example.

\subsection{GENERIC}

Grmela and Öttinger [40,69] put forward a general formalism to model systems that are locally not in equilibrium with respect to transformation. The premise of Grmela and Öttinger [40] is that the evolution of the state variables of an isolated system can be written as

$\frac{\mathrm{d} \boldsymbol{x}}{\mathrm{d} t}=\mathcal{L} \cdot \frac{\partial e}{\partial \boldsymbol{x}}+\mathcal{M} \cdot \frac{\partial s}{\partial \boldsymbol{x}}$

where $\boldsymbol{x}$ is the (column) vector of the state variables that describe the system, and $e$ and $s$ are the energy and the entropy densities of the system; in Eq. (60) the Volterra functional derivative of the total energy and entropy have been replaced by partial derivatives of their densities without 
loss of generality if the energy and entropy densities of the system depend on the state variables but not on their spatial gradients. The matrix $\mathcal{L}$ is antisymmetric, $(\boldsymbol{a} \cdot \mathcal{L}=-\mathcal{L}$. $\boldsymbol{a} \forall \boldsymbol{a})$ and represents the reversible dynamics of the system, whereas the matrix $\mathcal{M}$ is symmetric and positive definite $(\boldsymbol{a} \cdot \mathcal{M}=\mathcal{M} \cdot \boldsymbol{a} ; \boldsymbol{a} \cdot \mathcal{M} \cdot \boldsymbol{a} \geq 0 \forall \boldsymbol{a})$ and represents the irreversible dynamics of the system. Moreover, the matrices $\mathcal{L}$ and $\mathcal{M}$ enjoy the properties

$\mathcal{L} \cdot \frac{\partial s}{\partial \boldsymbol{x}} \equiv-\frac{\partial s}{\partial \boldsymbol{x}} \cdot \mathcal{L}=\mathbf{0}$

$\mathcal{M} \cdot \frac{\partial e}{\partial \boldsymbol{x}} \equiv \frac{\partial e}{\partial \boldsymbol{x}} \cdot \mathcal{M}=\mathbf{0}$

i.e., the gradient of the entropy with respect to the state variables is always in the null space of $\mathcal{L}$, and the gradient of the energy with respect to the state variables is always in the null space of $\mathcal{M}$. This property guarantees that the principle of conservation of energy and the local form of the second law of thermodynamics are always satisfied,

$\dot{e}=\frac{\partial e}{\partial \boldsymbol{x}} \cdot \frac{\mathrm{d} \boldsymbol{x}}{\mathrm{d} t}=\frac{\partial e}{\partial \boldsymbol{x}} \cdot \mathcal{L} \cdot \frac{\partial e}{\partial \boldsymbol{x}}+\frac{\partial e}{\partial \boldsymbol{x}} \cdot \mathcal{M} \cdot \frac{\partial s}{\partial \boldsymbol{x}}=0$

$\dot{s}=\frac{\partial s}{\partial \boldsymbol{x}} \cdot \frac{\mathrm{d} \boldsymbol{x}}{\mathrm{d} t}=\frac{\partial s}{\partial \boldsymbol{x}} \cdot \mathcal{L} \cdot \frac{\partial e}{\partial \boldsymbol{x}}+\frac{\partial s}{\partial \boldsymbol{x}} \cdot \mathcal{M} \cdot \frac{\partial s}{\partial \boldsymbol{x}} \geq 0$.

The GENERIC framework is very general and has been applied to a number of systems both at the macroscopic and microscopic level. The chief difference between GENERIC and the Generalized Bracket approach is that GENERIC is a "double generator" formalism (i.e., it employs separately energy and entropy in the fundamental Eq. (60)) which is applicable to situations where no concept of temperature is available. The relationships of GENERIC to the Matrix Model and Generalized Bracket approach have been examined by Edwards et al. [102], Edwards [103] and Jongschaap [104]. When applied to macroscopic models, the operator formulation of GENERIC requires simpler algebra than the Bracket approach. Whereas at the microscopic level some of the symmetries of the matrices $\mathcal{L}$ and $\mathcal{M}$ can be derived (for example, the Onsager relations), it is not clear yet to the authors whether such symmetries must hold at all levels of description-specifically at the macroscopic level, and when the matrices $\mathcal{L}$ and $\mathcal{M}$ are allowed to depend on the state variables $\boldsymbol{x}$. One notable advantage of GENERIC is that it is invariant to a nonlinear change of state variables $\boldsymbol{y}=\boldsymbol{f}(\boldsymbol{x})$, provided that the mapping $\boldsymbol{f}$ is one-to-one (bijective)-i.e., the Jacobian of the mapping $\mathcal{J} \equiv \partial \boldsymbol{f} / \partial \boldsymbol{x}$ is invertible everywhere. (This addresses a long-standing criticism of the Onsager relations by Truesdell [105].) This can be verified easily because the new state variables $y$ obey the dynamical equation

$\frac{\mathrm{d} \boldsymbol{y}}{\mathrm{d} t}=\tilde{\mathcal{L}} \cdot \frac{\partial e}{\partial \boldsymbol{y}}+\tilde{\mathcal{M}} \cdot \frac{\partial s}{\partial \boldsymbol{y}}$,

where $\tilde{\mathcal{L}} \equiv \mathcal{J} \mathcal{L} \mathcal{J}^{\mathrm{T}}$ is skew-symmetric iff $\mathcal{L}$ is skew-symmetric, and $\tilde{\mathcal{M}} \equiv \mathcal{J} \mathcal{M} \mathcal{J}^{\mathrm{T}}$ is symmetric and positive definite iff $\mathcal{M}$ is so, provided that $\mathcal{J}$ is invertible.

\subsection{Summary of the literature}

Leonov's approach (Section 3.1), the Generalized Bracket approach (Section 3.2), and the Matrix Model (Section 3.3) lead to identical results when applied to incompressible, isothermal, non-diffusing flows of polymeric liquids described by an explicit polymer conformation variable, except that in Leonov's theory the expression of the elastic stress is postulated and not derived; this excludes from Leonov's theory some models which are admissible in the Generalized Bracket and Matrix Model-for example, the partially retracting non-affine motion of Larson [106]. Moreover, it is not clear how to extend Leonov's ideas to include explicitly the entanglement density in the set of state variables.

The Generalized Bracket formalism, the Matrix Model, and GENERIC can be extended to include the entanglement density in the state variables (for example, a brief analysis of theories that account explicitly for entanglements can be found in Sec. 8.2.2-C of [2], and a more recent theory which accounts for a tensor and a scalar variable has been reported by Öttinger [107]). Yet, it is not evident whether the symmetries that are implicit in the Generalized Bracket (Section 3.2), in the Matrix Model (Section 3.3), and in GENERIC should be imposed on the equation set.

The structure underlying the Generalized Bracket approach, the Matrix Model, and GENERIC is that of linear irreversible thermodynamics, even though non-linear effects have been introduced into all approaches-for example, the tensors $\boldsymbol{\Lambda}$ in the bracket approach (Eq. (38)) and the Matrix Model (Eq. (54)) and the matrix $\mathcal{M}$ in GENERIC (Eq. (60)) can depend on the conformation tensor. More specifically, the equations of change of microstructure are assumed to depend linearly on the velocity gradient. The currently available models of interaction between flow and polymer conformation happen to be linear in the velocity gradient (Section 7.2); therefore, it is not surprising that the Generalized Bracket and Matrix Model successfully generalized the theories of flow of polymeric liquids with a conformation variable. The published models of interaction of flow and entanglements either assume that the entanglement and disentanglement processes are independent of flow, or assume a nonlinear relationship between entanglement generation and velocity gradient: it is not clear to the authors whether the Generalized Bracket approach, Matrix Model, and GENERIC can accommodate these modes of interaction between flow and entanglements.

A different approach to the mesoscopic modeling of microstructured liquids is presented here. It extends classical continuum thermodynamics based on the hypothesis of local equilibrium, but assumes no intrinsic symmetries in the transport equations of microstructure. The physical principles and assumptions needed to arrive at the results of Leonov, Grmela, Beris and Edwards, Jongschaap et al., and Grmela and Öttinger are identified, and the modeling is ex- 
tended to include the effect of changing entanglement density on the flow of polymer solutions and melts. ${ }^{3}$

\section{Thermodynamics with microstructural variables}

Macroscopic thermodynamics deals with equilibrium states in which the state variables of a system do not change, and equilibrium processes in which the state variables change so slowly that at any instant the system is approximately in an equilibrium state. For this to be possible, on the time and length scales of observation spatial gradients of the state variables of the thermodynamic system must be very small, and the microstructure and the chemical composition of the material must equilibrate rapidly to their natural states. If finite gradients of the state variables occur, then the system is not in equilibrium with respect to transport; if the microstructure or chemical composition change in time when gradients are absent, then the system is not in equilibrium with respect to transformation. This latter case includes chemically reacting systems; it also includes systems in which shrinkage or swelling is delayed upon change in temperature or composition.

Macroscopic thermodynamics is used with extraordinarily few exceptions to relate local properties, even when there are gradients and when the system is undergoing transformation: the tremendously useful condition of local equilibrium, introduced by Gibbs [108], is at the foundation of the whole thermodynamics of continua and of the theory of transport phenomena. The local equilibrium hypothesis says that ([43], Ch. 3)

... although the total system is not in [thermodynamic] equilibrium, there exists within small mass elements a state of "local" [thermodynamic] equilibrium, for which the local entropy $s$ is the same function $\left[s=s\left(u, v, c_{k}\right)\right]$ of $u$ [internal energy], $v$ [specific volume] and $c_{k}$ [mass fractions] as in real [thermodynamic] equilibrium.

This relationship between the state variables is called Gibbs' fundamental equation.

In low-molecular weight, non-reacting liquids and gases, the assumption of local equilibrium implies that an infinitesimal element of mass would be at equilibrium (i.e., the value of the state variables would not change) if it were isolated from the system by rigid, impermeable, adiabatic walls, i.e., the value of the state variables in the infinitesimal mass element would be constant in time (Fig. 2(a)). In particular, the entropy of the mass element would not change in time, and no irreversible processes would occur. This interpretation of local equilibrium must be altered when chemically reacting, low-molecular weight liquids and gases are considered. In an isolated infinitesimal mass element of the system, the mass

\footnotetext{
${ }^{3}$ The mutual relationships of the different approaches to microstructure modeling and their ties with other thermodynamic theories are diagrammed in Fig 1.3 of [60].
}
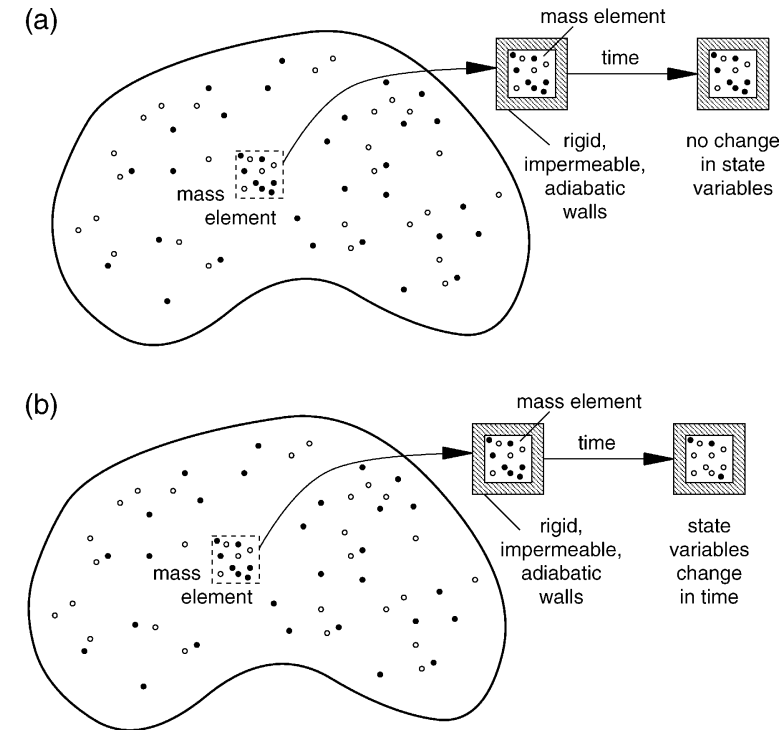

Fig. 2. Non-reacting (a) vs. reacting (b) systems.

fractions of the reacting chemical species change in time (Fig. 2(b)) until the reactions reach equilibrium: the mass element evolves spontaneously to a new state. The entropy of the mass element grows in time, and the assumption of local equilibrium means that the relationship between entropy and state variables holds even though irreversible processes are occurring due to the chemical reactions. Of course, the choice of a time scale is implicit in the definition of equilibrium with respect to transformation. For example, a mixture of hydrogen and oxygen at standard temperature and pressure is not at thermodynamic equilibrium with respect to reaction; but, absent a spark, such a mixture is changing so slowly that for most purposes it can be taken as in thermodynamic equilibrium.

Microstructured liquids are similar to chemically reacting systems. The microstructure of the liquid is often displaced from its equilibrium state during flow. If a small mass element of the flowing liquid were isolated from its surroundings, it would not be at equilibrium with respect to transformation: its microstructure would evolve in time to a natural state, and the state of the system would not change spontaneously thereafter. The characteristic time scale of microstructural evolution is frequently longer than the shortest timescales of practical interest in a process or flow, so that the microstructure cannot relax completely on the faster time scale of interest. The same can be true of microstructural relaxation required for shrinkage or swelling upon rapid change in temperature or composition.

This behavior of microstructured liquids can be exemplified by the elastic recovery of a polymeric liquid (melt or solution) following a sudden shear strain and subsequent removal of the boundary condition responsible for that strain. When a sudden shear strain is imposed on a polymeric liquid, the macroscopic deformation displaces the polymer molecules from their equilibrium distribution of conformations, and this induces a measurable non-isotropic stress: the 
local deformed configuration of the liquid differs from its local stress-free state-i.e., free of all stress, including elastic, or recoverable stress. If after the straining the boundary of the liquid is held in place, the polymer molecules rearrange spontaneously, and the non-isotropic stress relaxes in time to its isotropic equilibrium value; during this relaxation, the local stress-free state of the liquid evolves towards the local deformed state. If the boundary is freed after a long time, the liquid remains in its sheared configuration because the polymer molecules have rearranged and the sheared configuration has become the new stress-free state.

However, if the boundary of the liquid is freed after a time shorter than that needed for a complete rearrangement of the polymer molecules, the sheared configuration does not coincide with the stress-free state: the liquid evolves by essentially elastic displacement (recovery) to a stress-free configuration which is intermediate between the initial configuration at rest and the sheared configuration. This phenomenon, known as recoil $[4,109,110]$, was observed as early as a century ago by Trouton and Andrews [111] and Fano [112]. Interestingly, Trouton and Andrews connected recoil with the ability of a material (pitch) to store elastic energy:

$[\ldots]$ on removing the stress there is a flow back in the opposite direction, which gradually diminishes to zero with time. [...] Evidently, to do this, energy must have been stored in the substance in the form of elastic strain. [...] a store of elastic energy is gradually accumulated, which is preserved intact during the state of steady rotation, and is given out on removal of the stress to produce the return flow.

A thought experiment can be constructed where the shear is imposed in a vanishingly short time, and then the liquid is freed immediately. In this limiting process, the polymer molecules have no time to rearrange after the straining, and the stress-free state remains the initial unsheared configuration: the shear stress in the liquid is wholly recoverable, or elastic, and the liquid returns back to its initial configuration, like an elastomeric crosslinked rubber.

Material and process time scales are particularly important in a thermodynamic theory of processes of microstructured materials because a well-defined separation of material and process time scales is rarely possible. The role of time scales in process thermodynamics is thoroughly analyzed by Woods [113], with particular reference to internal variables. The basic idea used here (after [113]) to introduce microstructure in the thermodynamics of microstructured liquids is that there is a time scale $\lambda_{\mathrm{m}}$ that can be used to separate "fast" dissipative processes like viscous flow, and "slow" dissipative processes like the rearrangement of macromolecules in a flowing liquid, and that the slow dissipative processes can be considered conservative on the time scale $\lambda_{\mathrm{m}}$.

The local equilibrium hypothesis is assumed to hold on that time scale $\lambda_{\mathrm{m}}$, and the microstructural variables whose characteristic relaxation time is larger than $\lambda_{m}$ are included in Gibbs' equation; therefore, work done on the liquid to displace the microstructural variables from their equilibrium values is completely reversible on time scales much shorter than $\lambda_{\mathrm{m}}$, and completely irreversible on time scales much longer than $\lambda_{\mathrm{m}}$. Conversely, microstructural processes that relax faster than $\lambda_{\mathrm{m}}$ are dissipative, whereas microstructural processes that relax slower than $\lambda_{\mathrm{m}}$ are reversible (on short time scales) and contribute to reversible work storage.

An additional restriction is placed on the microstructural variables, that they should be extensive variables. Extensive variables are preferred to diffusive fluxes-which are used in extended irreversible thermodynamics $[72,114,77]$ - for consistency with Gibbs' [108] original postulate that the internal energy of a system is a homogeneous function of all extensive variables ([see also [113], p. 14 and 29]). Moreover, any extensive variable per unit volume $\Phi$ obeys a transport equation of the type

$$
\underbrace{\frac{\partial \Phi}{\partial t}}_{\text {accumulation }}=-\nabla \cdot(\underbrace{v \Phi}_{\text {convection }}+\underbrace{\boldsymbol{J}_{\Phi}}_{\text {diffusion }})+\underbrace{\Phi_{\mathrm{g}}}_{\text {generation }},
$$

where $\boldsymbol{v}$ is the liquid's velocity, $\boldsymbol{J}_{\Phi}$ is the diffusive flux of $\Phi$, and $\Phi_{\mathrm{g}}$ is the local volumetric rate of generation of $\Phi$. Thus, it is convenient to write the transport equations in terms of extensive variables per unit volume. Incidentally, such a form of transport equation is illustrative when deriving models because it separates clearly the effects of transport (flux term) and transformation (generation term) which can be linked directly to microscopic models. ${ }^{4}$

The local form of the second law of thermodynamics requires a non-negative generation term in the entropy balance equation, and this condition sets constraints on the expressions of the diffusive fluxes and the generation terms in all balance equations (see also the landmark paper by Liu [115]). These constraints alone simplify only slightly the constitutive equations of the diffusive fluxes and the generation terms. However, important simplifications and conclusions can be derived by further assuming that the diffusive fluxes should be linear functions of the thermodynamic affinities, which are the spatial gradients of the conjugate thermodynamic variables - the derivatives of the internal energy with respect to the thermodynamics state variables. This assumption has proven adequate to describe flow and transport in low molecular weight liquids and gases [43], but its applicability to transport in macromolecular liquids has not been confirmed experimentally yet.

Further constraints on the constitutive equations and on the possible coupling of the diffusive fluxes with the the thermodynamic affinities can be obtained by invoking isotropy, objectivity and frame indifference. These princi-

\footnotetext{
${ }^{4}$ Eq. (66) is also a useful framework for developing computational codes based on finite elements and (even more so) finite volume.
} 
ples are sometimes misused [113], and their applicability depends on the particular microstructural material in hand.

The following algorithm (the idea of an algorithm is borrowed from the work of Kuiken [116] on thermodynamics applied to linear viscoelasticity) summarizes the steps leading to a theory of flow and transport in liquids with relaxing microstructure consistent with continuum thermodynamics; the detailed derivation is presented in Section 5.

Select a time scale $\lambda_{\mathrm{m}}$ that is appropriate to the material and the process

Identify microstructural variables that cannot relax on the time scale $\lambda_{\mathrm{m}}$

Apply the principle of objectivity: microstructural variables must be independent of the frame of reference in which time derivatives are evaluated, i.e., they must be objective scalars, vectors, dyadics, or higher rank tensors

Recast microstructural variables as extensive variables

Write Gibbs' equation: internal energy $u$ is a function of all extensive variables $x_{i}: u=u\left(x_{1}, \ldots, x_{N}\right)$

Write transport equations of all extensive variables per unit volume

Couple the transport equations with Gibbs' equation and compute the local rate of entropy generation $s_{\mathrm{g}}$

Simplify transport equations if appropriate (e.g. neglect diffusive fluxes)

Enforce $s_{\mathrm{g}} \geq 0$ for all possible processes (local entropy production inequality)

Always appropriate

- Use representation theorems on tensor-valued isotropic functions of tensors to build general constitutive equations of the fluxes and the generation terms

- Apply the principle of indifference to the choice of any rotating rigid frame: $s_{\mathrm{g}}$ must not depend on the rigid, rotating frame with respect to which time derivatives are evaluated

\section{Sometimes appropriate}

- Postulate linear relationship between diffusive fluxes and thermodynamic affinities

- Postulate linear relationship between generation terms and thermodynamic affinities

- Break thermodynamic affinities into orthogonal, i.e., independent, parts ([43], p. 58)

- Assign time-reversal (motion-reversal) parities to diffusive fluxes and generation terms, i.e., establish which fluxes and generation terms would change sign (odd parity) and which would retain their sign (even parity) if the velocity of all molecules was suddenly reversed; derive Onsager's reciprocal relations ([113], p. 163 ff.)
Derive relationships between diffusive fluxes from local entropy production inequality

Derive relationship between elastic stress and state variables from the local entropy production inequality

Derive constraints on the constitutive equations of the fluxes and generation terms from the local entropy production inequality.

\section{Application to polymeric liquids}

The polymeric liquid considered here is a one-component, compressible, non-reacting continuum, i.e., the fluctuations in expectations are negligible compared to their means. The local form of the balance equations of mass, momentum, mechanical energy, internal energy, and entropy in the absence of electromagnetic fields is

$$
\begin{aligned}
& \frac{\partial \rho}{\partial t}=-\nabla \cdot \rho \boldsymbol{v} \\
& \frac{\partial}{\partial t} \rho \boldsymbol{v}=-\nabla \cdot(\boldsymbol{v} \rho \boldsymbol{v}-\boldsymbol{T})-\rho \nabla \Theta \\
& \frac{\partial}{\partial t} \rho\left(\frac{1}{2} v^{2}+\Theta\right) \\
& \quad=-\nabla \cdot\left(\boldsymbol{v} \rho\left(\frac{1}{2} v^{2}+\Theta\right)-\boldsymbol{T} \cdot \boldsymbol{v}\right)-\boldsymbol{T}^{\mathrm{T}}: \nabla \boldsymbol{v} \\
& \frac{\partial}{\partial t} \rho u=-\nabla \cdot\left(\boldsymbol{v} \rho u+\boldsymbol{J}_{\mathrm{u}}\right)+\boldsymbol{T}^{\mathrm{T}}: \nabla \boldsymbol{v} \\
& \frac{\partial}{\partial t} \rho s=-\nabla \cdot\left(\boldsymbol{v} \rho s+\boldsymbol{J}_{\mathrm{s}}\right)+s_{\mathrm{g}} .
\end{aligned}
$$

Here $\rho$ is density, $v$ is the magnitude of the velocity vector $\boldsymbol{v}, \Theta$ is the potential energy per unit mass due to stationary external fields such as gravity, $\boldsymbol{T}$ is the total (Cauchy) stress dyadic, $\nabla$ is the usual gradient operator in physical space $(\nabla=\partial / \partial \boldsymbol{x}), u$ is the internal energy per unit mass, $\boldsymbol{J}_{\mathrm{u}}$ is the diffusive flux of internal energy, $s$ is the (total) entropy per unit mass, $\boldsymbol{J}_{\mathrm{S}}$ is the diffusive flux of entropy, and $s_{\mathrm{g}}$ is the local volumetric rate of entropy generation. ${ }^{5}$

The polymeric liquid is assumed to be in equilibrium for an observer who examines it on a time scale much

\footnotetext{
${ }^{5}$ The total entropy of an ideal rubber decreases during isothermal deformations, yet $s_{\mathrm{g}}=0$. During the isothermal deformation of a rubber, the internal energy stays constant even though the environment is doing work on the rubber; thus, there is a positive flux of heat (i.e., entropy) from the rubber into the environment. Such heat flux is responsible for the decrease in the rubber's configurational entropy; because the temperature stays constant, the vibrational entropy of the rubber does not change. In an adiabatic deformation of a rubber, the total entropy of the rubber stays constant, while at the same time the temperature grows. The temperature increase is accompanied by an increase in vibrational entropy which balances the drop in configurational entropy caused by the deformation [117].
} 
shorter than the characteristic relaxation time of the macromolecules. The fundamental equation has to account therefore for the relevant non-relaxed molecular processes. The two important phenomena are the stretch and orientation (conformation) of the molecules and the formation and destruction of entanglements. The latter phenomenon is relevant when the liquid's concentration is high enough that entanglements between polymer molecules are important $[8,10]$ - equivalently, if the motion of each polymer molecule is confined to a tube-like region by the presence of surrounding molecules [11]. The former can (approximately) be accounted for by a symmetric and positive-definite dyadic $\boldsymbol{M}$, the conformation dyadic; the latter, by a scalar $e$, the number of entanglements per unit mass. In the case of rod-like polymers, molecular stretching is less relevant and the conformation dyadic can often be taken to have constant trace [2,11]. At high enough concentration, rod-like polymers order spontaneously into liquid crystalline mesophases, and this ordering must be accounted for [2]. The treatment below is restricted to liquids which are isotropic in the absence of flow.

In a molecular theory of polymeric liquids, at each point in space the liquid is fully characterized once the expectation value of the distribution function $\Psi(\boldsymbol{r}, \boldsymbol{x}, t)$ of segments $\boldsymbol{r}$ is given, where $\Psi(\boldsymbol{r}, \boldsymbol{x}, t) \mathrm{d} \boldsymbol{r} \mathrm{d} \boldsymbol{x}$ is the number of segments per unit mass of liquid whose end-to-end distance falls between $\boldsymbol{r}$ and $\boldsymbol{r}+\mathrm{d} \boldsymbol{r}$, and whose center of mass is between $\boldsymbol{x}$ and $\boldsymbol{x}+\mathrm{d} \boldsymbol{x}$. In dilute solution theories, $\boldsymbol{r}$ is usually the coil's end-to-end distance; in theories of entangled solutions $\boldsymbol{r}$ is the distance between successive entanglements on the same coil; in theories of crosslinked rubbers, $\boldsymbol{r}$ is the distance between consecutive crosslinks. In such a detailed theory all the thermodynamic functions of the material element located at the position in space $\boldsymbol{x}$ at time $t$ can be calculated from the knowledge of $\Psi(\boldsymbol{r}, \boldsymbol{x}, t)$. In this case $\boldsymbol{M}$ can be defined in terms of the distribution function $\Psi(\boldsymbol{r})$ as

$\boldsymbol{M}(\boldsymbol{x}, t)=\int_{\boldsymbol{r} \in R^{3}} \mathrm{~d} \boldsymbol{r} \Psi(\boldsymbol{r}, \boldsymbol{x}, t) \boldsymbol{r} \boldsymbol{r}$.

The information carried by $\boldsymbol{M}$ is not all of that carried by $\Psi$; therefore, a theory that relies on $\boldsymbol{M}$ in place of $\Psi$ is not as detailed. Still, the molecular theories developed to describe the behavior of polymeric liquids [12] relate the elastic stress dyadic $\sigma$ to the second moment of $\Psi$ and it is reasonable to expect that a single dyadic-valued variable and the entanglement concentration may describe adequately the internal state of the flowing liquid. (However, in more detailed models a coil is described by a chain of connected beads, and $\boldsymbol{r}$ represents the connector of two consecutive beads.) Öttinger and Grmela ([69], p. 6648) remark that a thermodynamic description in terms of the second moment of the distribution function "can only be valid if all the higher moments either are functions of the second moment or possess a rapid time evolution." Of course, this is always true when one tries to reduce the number of variables used to describe any dynamical system - the dynamics of the variables that are eliminated must either be fast compared to the dynamics of the reduced variables or else they must be expressible (at least with reasonable accuracy) in terms of the reduced variables alone.

The extensive variables that characterize the thermodynamic state of a fluid element are therefore the specific internal energy $u$, specific entropy $s$, specific volume $v \equiv \rho^{-1}$, augmented by conformation per unit mass $\boldsymbol{M}$, and number of entanglements per unit mass $e$. The conformation dyadic can also be expressed in terms of the normalized distribution function $\psi(\boldsymbol{r}) \equiv \Psi(\boldsymbol{r}) / c_{\mathrm{p}}$ by

$\boldsymbol{M}=c_{\mathrm{p}} \int_{\boldsymbol{r} \in R^{3}} \mathrm{~d} \boldsymbol{r} \psi(\boldsymbol{r}, \boldsymbol{x}, t) \boldsymbol{r} \boldsymbol{r} \equiv c_{\mathrm{p}}\langle\boldsymbol{r} \boldsymbol{r}\rangle$

where

$c_{\mathrm{p}} \equiv \int_{\boldsymbol{r} \in R^{3}} \mathrm{~d} \boldsymbol{r} \Psi(\boldsymbol{r}, \boldsymbol{x}, t)$

is the number of polymer segments per unit mass of the liquid. Under the hypothesis of local equilibrium, Gibbs' fundamental equation of thermodynamics that relates all extensive quantities per unit mass is

$u=u(s, v, \boldsymbol{M}, e)$.

The general transport equations of microstructure are

$\frac{\partial}{\partial t} \rho e=-\nabla \cdot\left(\boldsymbol{v} \rho e+J_{\mathrm{e}}\right)+e_{\mathrm{g}}$

$\frac{\partial}{\partial t} \rho \boldsymbol{M}=-\nabla \cdot\left(\boldsymbol{v} \rho \boldsymbol{M}+\boldsymbol{J}_{\boldsymbol{M}}\right)+\boldsymbol{M}_{\mathrm{g}}$

where $\boldsymbol{J}_{\mathrm{e}}$ and $\boldsymbol{J}_{\boldsymbol{M}}$ are the diffusive fluxes of entanglements and conformation and $e_{\mathrm{g}}$ and $\boldsymbol{M}_{\mathrm{g}}$ are the local volumetric rates of generation of entanglements and conformation. Because $\boldsymbol{M}$ is a symmetric tensor, the diffusive flux $\boldsymbol{J}_{\boldsymbol{M}}$ must be symmetric with respect to transposition of its last two indices, i.e., $\boldsymbol{J}_{\boldsymbol{M} i j k}=\boldsymbol{J}_{\boldsymbol{M} i k j}$, and the rate of generation $\boldsymbol{M}_{\mathrm{g}}$ must be symmetric, $\boldsymbol{M}_{\mathrm{g}}=\boldsymbol{M}_{\mathrm{g}}^{\mathrm{T}}$.

The transport equations of thermodynamic variables can be rewritten as

$\dot{v}=v \nabla \cdot \boldsymbol{v}$

$\rho \dot{u}=-\nabla \cdot J_{\mathrm{u}}+\boldsymbol{T}: \nabla \boldsymbol{v}$

$\rho \dot{s}=-\nabla \cdot \boldsymbol{J}_{\mathrm{s}}+s_{\mathrm{g}}$

$\rho \dot{e}=-\nabla \cdot J_{\mathrm{e}}+e_{\mathrm{g}}$

$\rho \dot{\boldsymbol{M}}=-\nabla \cdot \boldsymbol{J}_{\boldsymbol{M}}+\boldsymbol{M}_{\mathrm{g}}$,

where the over-dot stands for the material (substantial) time derivative, $\dot{u} \equiv \partial u / \partial t+\boldsymbol{v} \cdot \nabla u$. It follows from Gibbs' Eq. (75) that

$\dot{u}=\frac{\partial u}{\partial s} \dot{s}+\frac{\partial u}{\partial v} \dot{v}+\frac{\partial u}{\partial e} \dot{e}+\frac{\partial u}{\partial \boldsymbol{M}}: \dot{\boldsymbol{M}}^{\mathrm{T}}$ $\equiv T \dot{s}+\tilde{\pi} \dot{v}+\epsilon \dot{e}+S: \dot{M}^{\mathrm{T}}$,

where $T \equiv \partial u / \partial s>0$ is defined to be the thermodynamic temperature, $-\tilde{\pi} \equiv-\partial u / \partial v$ is the thermodynamic 
pressure, ${ }^{6}$ and $\epsilon \equiv \partial u / \partial e$ and $\boldsymbol{S} \equiv \partial u / \partial \boldsymbol{M}$ are the conjugate variables of entanglement and conformation density, hereafter termed microstructural affinities.

Multiplying Eq. (83) by the density, using the balance equations, the symmetry of $\dot{\boldsymbol{M}}$, and the identities

$T \nabla \cdot \boldsymbol{J}_{\mathrm{s}}=\nabla \cdot\left(T \boldsymbol{J}_{\mathrm{s}}\right)-\nabla T \cdot \boldsymbol{J}_{\mathrm{s}}$

$\boldsymbol{S}: \nabla \cdot \boldsymbol{J}_{\boldsymbol{M}}=\nabla \cdot\left(\boldsymbol{J}_{\boldsymbol{M}}: \boldsymbol{S}\right)-\nabla \boldsymbol{S} \bullet \boldsymbol{J}_{\boldsymbol{M}}$,

yields the expression of the entropy production rate:

$$
\begin{aligned}
T s_{\mathrm{g}}= & \nabla \cdot\left(-\boldsymbol{J}_{\mathrm{u}}+T \boldsymbol{J}_{\mathrm{s}}+\epsilon \boldsymbol{J}_{\mathrm{e}}+\boldsymbol{J}_{\boldsymbol{M}}: \boldsymbol{S}\right)+(\boldsymbol{T}-\tilde{\pi} \boldsymbol{I}): \nabla \boldsymbol{v} \\
& -\left(\nabla T \cdot \boldsymbol{J}_{\mathrm{s}}+\nabla \epsilon \cdot \boldsymbol{J}_{\mathrm{e}}+\nabla \boldsymbol{S} \bullet \boldsymbol{J}_{\boldsymbol{M}}\right)-\left(\epsilon e_{\mathrm{g}}+\boldsymbol{S}: \boldsymbol{M}_{\mathrm{g}}\right)
\end{aligned}
$$

The second law of thermodynamics requires that $s_{\mathrm{g}} \geq 0$ in any admissible process satisfying the balance laws, Eqs. (78)-(82). This local statement of the second law of thermodynamics is hereafter termed local entropy production inequality; in the absence of microstructural variables, the local entropy production inequality is equivalent to the local Clausius-Duhem inequality used in rational thermodynamics ([118], p. 295).

The first term in Eq. (86) is the divergence of a flux; therefore, it is not definite in sign and must vanish, leading to the relationship between the diffusive fluxes

$\boldsymbol{J}_{\mathrm{u}}=T \boldsymbol{J}_{\mathrm{s}}+\epsilon \boldsymbol{J}_{\mathrm{e}}+\boldsymbol{J}_{\boldsymbol{M}}: \boldsymbol{S}$,

(of course, $\boldsymbol{J}_{\mathrm{u}}$ is determined only within a divergence-free term) and the expression of the entropy production rate

$$
\begin{aligned}
T S_{\mathrm{g}}= & (\boldsymbol{T}-\tilde{\pi} \boldsymbol{I}): \nabla \boldsymbol{v}-\left(\nabla T \cdot \boldsymbol{J}_{\mathrm{s}}+\nabla \epsilon \cdot \boldsymbol{J}_{\mathrm{e}}+\nabla \boldsymbol{S} \bullet \boldsymbol{J}_{\boldsymbol{M}}\right) \\
& -\left(\epsilon_{\mathrm{g}}+\boldsymbol{S}: \boldsymbol{M}_{\mathrm{g}}\right) .
\end{aligned}
$$

Not all the terms in Eq. (88) necessarily produce entropy: some terms might give a negative contribution to the rate of production of entropy which is balanced exactly by other terms in Eq. (88). It does not seem possible to extract any more information from Eq. (88) without invoking additional principles (like the principle of macroscopic time reversal, Section 3.3 and [3]) or making further assumptions on the form of the constitutive equations of the fluxes and generation terms; therefore, Eq. (88) should be used to check if general nonlinear constitutive equations obey the second law of thermodynamics.

\subsection{Quasilinear phenomenological laws}

Important simplifications can be introduced by assuming that the constitutive equations of the fluxes $\boldsymbol{T}, \boldsymbol{J}_{\mathrm{s}}, \boldsymbol{J}_{\mathrm{e}}, \boldsymbol{J}_{\boldsymbol{M}}$ and of the generation terms $e_{\mathrm{g}}, \boldsymbol{M}_{\mathrm{g}}$ are linear functions of the velocity gradient $\nabla \boldsymbol{v}$ and of the thermodynamic affinities $\nabla T, \nabla \epsilon, \nabla \boldsymbol{S}$. This assumption is not very restrictive because

\footnotetext{
${ }^{6}$ The negative sign comes from the convention in thermodynamics that compression is positive and tension is negative.
}

the constitutive equations can still be non-linear functions of the state variables $s, v, e, \boldsymbol{M}$. Such constitutive equations are called quasilinear phenomenological laws, and are normally used in theories of transport phenomena. For example, Fourier's law of heat conduction says that the heat flux $J_{\mathrm{u}} \equiv-\kappa(\nu, T) \nabla T$ is a linear function of the gradient of temperature, but that it can depend arbitrarily on the temperature and on the specific volume (or the pressure) through the thermal conductivity $\kappa$ ([42], pp. 249-262)—provided that $\kappa \geq 0$. Similarly, Newton's law of viscosity says that the viscous stress must be linearly proportional to the rate of strain, but the viscosity coefficient can depend non-linearly on the temperature and specific volume or pressure ([42], pp. 15-29).

Taking constitutive equations of the generation terms that are linear functions of $\boldsymbol{S}$ and $\epsilon$ does not lead to significant simplifications, yet it restricts unnecessarily the range of admissible constitutive equations; therefore, this hypothesis is not adopted here. A similar situation arises in multicomponent reactive media, where the rate of reaction usually depends nonlinearly on the chemical affinities - the derivatives of the internal energy with respect to concentration at fixed entropy and specific volume-and the linear laws break down ([43], pp. 205-206).

The diffusive fluxes of entropy, entanglements, and conformation and the stress are each split into a term independent of the thermodynamic affinities (respectively $\boldsymbol{J}_{\mathrm{s}}^{0}, \boldsymbol{J}_{\mathrm{e}}^{0}$, $\boldsymbol{J}_{\boldsymbol{M}}^{0}$, and $\boldsymbol{\sigma}$ ) and a term linearly dependent on the thermodynamic affinities (for the stress $\boldsymbol{T} \equiv \boldsymbol{\sigma}+\boldsymbol{\tau}, \boldsymbol{\sigma}$ is the elastic, or non-dissipative part, and $\boldsymbol{\tau}$ is the viscous, or dissipative part):

$$
\boldsymbol{J}_{\mathrm{s}} \equiv \boldsymbol{J}_{\mathrm{s}}^{0}-\boldsymbol{L}^{s s} \cdot \nabla T-\boldsymbol{L}^{s e} \cdot \nabla \epsilon-\boldsymbol{L}^{s \boldsymbol{M}} \bullet \nabla \boldsymbol{S}-\boldsymbol{L}^{s \boldsymbol{v}}: \nabla \boldsymbol{v}
$$

$$
\boldsymbol{J}_{\mathrm{e}} \equiv \boldsymbol{J}_{\mathrm{e}}^{0}-\boldsymbol{L}^{e s} \cdot \nabla T-\boldsymbol{L}^{e e} \cdot \nabla \epsilon-\boldsymbol{L}^{e \boldsymbol{M}} \bullet \nabla \boldsymbol{S}-\boldsymbol{L}^{e \boldsymbol{v}}: \nabla \boldsymbol{v}
$$

$$
\begin{aligned}
& \boldsymbol{J}_{\boldsymbol{M}} \equiv \boldsymbol{J}_{\boldsymbol{M}}^{0}-\boldsymbol{L}^{\boldsymbol{M} s} \cdot \nabla T-\boldsymbol{L}^{\boldsymbol{M} e} \cdot \nabla \epsilon-\boldsymbol{L}^{\boldsymbol{M} \boldsymbol{M}} \bullet \nabla \boldsymbol{S} \\
& \quad-\boldsymbol{L}^{\boldsymbol{M} \boldsymbol{v}}: \nabla \boldsymbol{v} \\
& \boldsymbol{T} \equiv \boldsymbol{\sigma}+\boldsymbol{L}^{\boldsymbol{v} s} \cdot \nabla T+\boldsymbol{L}^{\boldsymbol{v} e} \cdot \nabla \epsilon+\boldsymbol{L}^{\boldsymbol{v M}} \bullet \nabla \boldsymbol{S}+\boldsymbol{L}^{\boldsymbol{v} \boldsymbol{v}}: \nabla \boldsymbol{v}
\end{aligned}
$$

$e_{\mathrm{g}} \equiv e_{\mathrm{g}}^{0}+\boldsymbol{r}^{e s} \cdot \nabla T+\boldsymbol{r}^{e e} \cdot \nabla \epsilon+\boldsymbol{R}^{e \boldsymbol{M}} \bullet \nabla \boldsymbol{S}+\boldsymbol{R}^{e \boldsymbol{v}}: \nabla \boldsymbol{v}$

$$
\begin{aligned}
\boldsymbol{M}_{\mathrm{g}} \equiv & \boldsymbol{M}_{\mathrm{g}}^{0}+\boldsymbol{R}^{\boldsymbol{M} s} \cdot \nabla T+\boldsymbol{R}^{\boldsymbol{M} e} \cdot \nabla \epsilon+\boldsymbol{R}^{\boldsymbol{M} \boldsymbol{M}} \bullet \nabla \boldsymbol{S} \\
& +\boldsymbol{R}^{\boldsymbol{M} \boldsymbol{v}}: \nabla \boldsymbol{v} .
\end{aligned}
$$

The tensors $\boldsymbol{L}^{s s}, \boldsymbol{L}^{s e}, \boldsymbol{L}^{s \boldsymbol{M}}, \ldots, \boldsymbol{R}^{\boldsymbol{M v}}$ are called phenomenological coefficients. The phenomenological coefficients denoted by $\boldsymbol{L}$ couple diffusive fluxes to affinities, whereas those 
denoted by $\boldsymbol{R}$ or $\boldsymbol{r}$ couple generation terms to affinities. The first superscript denotes the type of flux or generation term; the second, the affinity. The phenomenological coefficients $\boldsymbol{r}^{e s}, \boldsymbol{r}^{e e}$, as well as the fluxes $\boldsymbol{J}_{\mathrm{s}}^{0}$ and $\boldsymbol{J}_{\mathrm{e}}^{0}$ are first-rank tensors (vectors); $\boldsymbol{L}^{s s}, \boldsymbol{L}^{s e}, \boldsymbol{L}^{e s}, \boldsymbol{L}^{e e}, \boldsymbol{R}^{e v}$ are second-rank tensors (dyadics); $\boldsymbol{L}^{s v}, \boldsymbol{L}^{e v}, \boldsymbol{L}^{\boldsymbol{v} s}, \boldsymbol{L}^{\boldsymbol{v} e}, \boldsymbol{R}^{e \boldsymbol{M}}, \boldsymbol{R}^{\boldsymbol{M} s}, \boldsymbol{R}^{\boldsymbol{M} e}$, and $\boldsymbol{J}_{M}^{0}$ are third-rank tensors; $\boldsymbol{L}^{s \boldsymbol{M}}, \boldsymbol{L}^{e \boldsymbol{M}} \boldsymbol{L}^{\boldsymbol{M} s}, \boldsymbol{L}^{\boldsymbol{M} e}, \boldsymbol{L}^{v v}, \boldsymbol{R}^{\boldsymbol{M} v}$ are fourth-rank tensors; $\boldsymbol{L}^{\boldsymbol{M} \boldsymbol{v}}, \boldsymbol{L}^{\boldsymbol{v} \boldsymbol{M}}, \boldsymbol{R}^{\boldsymbol{M} \boldsymbol{M}}$ are fifth-rank tensors; and $\boldsymbol{L}^{\boldsymbol{M} \boldsymbol{M}}$ is a sixth-rank tensor. All the fluxes and generation terms are polar scalars, vectors, and tensors, i.e., they do not change sign if the handedness of the basis is changed (see [113] for a useful discussion). Because all the affinities in Eqs. (89)-(94) are also polar tensors, so must be the phenomenological coefficients. The phenomenological coefficients are independent of $\nabla T, \nabla \epsilon, \nabla \boldsymbol{S}$, and $\nabla \boldsymbol{v}$ and must be isotropic functions of the state variables $s, v, e, \boldsymbol{M}$.

No polar vector can be built from a combination of a symmetric second-rank polar tensor, polar scalars, and the isotropic tensors $\boldsymbol{I}$ (second-rank idemfactor, polar) and $\boldsymbol{\epsilon}$ (third-rank alternator, axial) [60]; therefore, $\boldsymbol{r}^{e s}=\mathbf{0}, \boldsymbol{r}^{e e}=$ $\mathbf{0}, \boldsymbol{J}_{\mathrm{s}}^{0}=\mathbf{0}$, and $\boldsymbol{J}_{\mathrm{e}}^{0}=\mathbf{0}$. Similarly, no polar third-rank or fifth-rank tensor can be built from a combination of the state variables and isotropic tensors; therefore, all the third-rank and fifth-rank tensors vanish. Consequently, in this system fluxes of even tensorial-rank do not couple with affinities of odd tensorial rank and fluxes of odd rank do not couple with affinities of even rank, because the only anisotropy of this system is represented by the second-rank, symmetric polar tensor $\boldsymbol{M} .^{7}$ This property of some systems is sometimes called Curie's Principle, even though it follows from isotropy and representation theorems [105]. Moreover, isotropy has the (quite intuitive) consequence that there cannot be fluxes of entropy, entanglements, and conformation in the absence of spatial gradients of temperature and microstructural affinities even in the presence of a macroscopic velocity gradient, whereas there can be a non-vanishing stress even in the absence of a velocity gradient.

The flux of conformation is symmetric with respect to transposition of the last two indices, $\boldsymbol{J}_{\boldsymbol{M} i j k}=\boldsymbol{J}_{\boldsymbol{M} i k j}$, and so is the conformational affinity $\nabla \boldsymbol{S}$ because $\boldsymbol{M}$ is symmetric, i.e., $\nabla_{j} \boldsymbol{S}_{k l}=\nabla_{j} \boldsymbol{S}_{l k}$; therefore, the phenomenological coefficients have the following symmetries: $\boldsymbol{L}_{i j k l}^{\boldsymbol{M} s}=\boldsymbol{L}_{i k j l}^{\boldsymbol{M} s}$; $\boldsymbol{L}_{i j k l}^{\boldsymbol{M} e}=\boldsymbol{L}_{i k j l}^{\boldsymbol{M} e} ; \boldsymbol{L}_{i j k l}^{s \boldsymbol{M}}=\boldsymbol{L}_{i j l k}^{s \boldsymbol{M}} ; \boldsymbol{L}_{i j k l}^{e \boldsymbol{M}}=\boldsymbol{L}_{i j l k}^{e \boldsymbol{M}} ; \boldsymbol{L}_{i j k l p q}^{M M}=\boldsymbol{L}_{i k j l p q}^{M M} ;$ $\boldsymbol{L}_{i j k l p q}^{\boldsymbol{M} \boldsymbol{M}}=\boldsymbol{L}_{i k j l q p}^{\boldsymbol{M} \boldsymbol{M}}$. The entropy production rate Eq. (88) reduces to

$$
\begin{aligned}
T s_{\mathrm{g}}= & \left\{\nabla T \cdot\left(\boldsymbol{L}^{s s} \cdot \nabla T+\boldsymbol{L}^{s e} \cdot \nabla \epsilon+\boldsymbol{L}^{s \boldsymbol{M}} \bullet \nabla \boldsymbol{S}\right)\right. \\
& +\nabla \epsilon \cdot\left(\boldsymbol{L}^{e s} \cdot \nabla T+\boldsymbol{L}^{e e} \cdot \nabla \epsilon+\boldsymbol{L}^{e \boldsymbol{M}} \bullet \nabla \boldsymbol{S}\right) \\
& \left.+\nabla \boldsymbol{S} \bullet\left(\boldsymbol{L}^{\boldsymbol{M} s} \cdot \nabla T+\boldsymbol{L}^{\boldsymbol{M e}} \cdot \nabla \epsilon+\boldsymbol{L}^{\boldsymbol{M M}} \bullet \nabla \boldsymbol{S}\right)\right\}
\end{aligned}
$$

\footnotetext{
${ }^{7}$ In case of anisotropic media, the tensors describing the anisotropy of the medium must be added to the list of variables that can be used in the constitutive equations for the phenomenological coefficients. Such tensors may introduce other couplings between the various thermodynamic variables.
}

$$
\begin{aligned}
& +\nabla \boldsymbol{v}: \boldsymbol{L}^{\boldsymbol{v} v}: \nabla \boldsymbol{v}+\left(\boldsymbol{\sigma}-\tilde{\pi} \boldsymbol{I}-\epsilon \boldsymbol{R}^{e v}-\boldsymbol{S}: \boldsymbol{R}^{\boldsymbol{M} \boldsymbol{v}}\right): \nabla \boldsymbol{v} \\
& -\left(\epsilon e_{\mathrm{g}}^{0}+\boldsymbol{S}: \boldsymbol{M}_{\mathrm{g}}^{0}\right) \geq 0
\end{aligned}
$$

for all possible values of $s, v, e, \boldsymbol{M}, \nabla T, \nabla \epsilon, \nabla \boldsymbol{S}, \nabla \boldsymbol{v}$. The variables $\nabla T, \nabla \epsilon$, and $\nabla S$ appear only in the first brace of Eq. (95); therefore, the diffusive processes driven by $\nabla T, \nabla \epsilon$, and $\nabla \boldsymbol{S}$ must produce entropy independently of momentum diffusion and of entanglement and conformation generation. Hence, in matrix form

$$
\begin{aligned}
& {\left[\nabla T \nabla \frac{\partial u}{\partial e} \nabla \frac{\partial u}{\partial \boldsymbol{M}}\right] \underbrace{\left[\begin{array}{lll}
\cdot \boldsymbol{L}^{s s} . & \cdot \boldsymbol{L}^{s e} \cdot & \cdot \boldsymbol{L}^{s \boldsymbol{M}} \bullet \\
\cdot \boldsymbol{L}^{e s} . & \cdot \boldsymbol{L}^{e e .} & \cdot \boldsymbol{L}^{e \boldsymbol{M}} \bullet \\
\bullet \boldsymbol{L}^{\boldsymbol{M} s} . & \bullet \boldsymbol{L}^{\boldsymbol{M e}} \cdot & \bullet \boldsymbol{L}^{\boldsymbol{M M}} \bullet
\end{array}\right]}_{\mathcal{L}}} \\
& \times\left[\begin{array}{c}
\nabla T \\
\nabla \frac{\partial u}{\partial e} \\
\nabla \frac{\partial u}{\partial \boldsymbol{M}}
\end{array}\right] \geq 0
\end{aligned}
$$

for all possible values of $\nabla T, \nabla \epsilon$, and $\nabla S$; thus the matrix $\mathcal{L}$ must be positive semidefinite. All the second-rank tensors $\boldsymbol{L}^{s s}, \boldsymbol{L}^{s e}, \boldsymbol{L}^{e s}$, and $\boldsymbol{L}^{e e}$ are symmetric because they are isotropic functions of the symmetric tensor $\boldsymbol{M}$ and the scalars $s, v, e$.

\subsection{Onsager's reciprocal relations}

The general applicability of Onsager's reciprocal relations is still a controversial issue $[105,113]$ and their use may or not be appropriate in this case. It is beyond the scope of this article to discuss whether Onsager's reciprocal relations should be used when they cannot be derived from microscopic considerations.

If Onsager's reciprocal relations hold, then the off-diagonal tensors in the matrix $\mathcal{L}$ are related ([113], p. 163). The parities of all the fluxes $\boldsymbol{J}_{\mathrm{s}}, \boldsymbol{J}_{\mathrm{e}}$, and $\boldsymbol{J}_{\boldsymbol{M}}$ under a macroscopic time-reversal is odd $(-1)$; therefore, the second-rank tensor $\boldsymbol{L}^{s e}=\boldsymbol{L}^{e s T}$, the fourth-rank tensors $\boldsymbol{L}_{i j k l}^{s \boldsymbol{M}}=\boldsymbol{L}_{l i j k}^{\boldsymbol{M} s}$, and $\boldsymbol{L}_{i j k l}^{e \boldsymbol{M}}=\boldsymbol{L}_{\text {lijk }}^{\boldsymbol{M} e}$, and the sixth-rank tensor $\boldsymbol{L}_{i j k p q r}^{\boldsymbol{M} \boldsymbol{M}}=\boldsymbol{L}_{p q r i j k}^{\boldsymbol{M M}}$, or, in invariant notation, $\boldsymbol{a} \cdot \boldsymbol{L}^{s \boldsymbol{M}} \vdots \boldsymbol{d c b}=\boldsymbol{d c b} \vdots \boldsymbol{L}^{\boldsymbol{M} s} \cdot \boldsymbol{a}$, $a \cdot L^{e M} \vdots d c b=d c b \vdots L^{M e} \cdot a$, and $a b c \vdots L^{M M}:=d e f=$ $\boldsymbol{d e f}: L^{M M}: a b c$ for any choice of the vectors $a, b, \ldots, f$. The cross-coupling terms relating the vector fluxes of entropy $\boldsymbol{J}_{\mathrm{S}}$ and entanglements $\boldsymbol{J}_{\mathrm{e}}$ to the third-rank tensor $\nabla \boldsymbol{S}$ need not vanish. Coupling terms of the form

$$
\begin{aligned}
\boldsymbol{L}_{i j k l}^{s \boldsymbol{M}}= & \boldsymbol{L}_{i j k l}^{\boldsymbol{M}_{\boldsymbol{s}}}=\alpha_{0}\left(\boldsymbol{\delta}_{i j} \boldsymbol{\delta}_{k l}+\boldsymbol{\delta}_{i k} \boldsymbol{\delta}_{j l}+\boldsymbol{\delta}_{i l} \boldsymbol{\delta}_{j k}\right) \\
& +\alpha_{1}\left(\boldsymbol{M}_{i j} \boldsymbol{M}_{k l}+\boldsymbol{M}_{i k} \boldsymbol{M}_{j l}+\boldsymbol{M}_{i l} \boldsymbol{M}_{j k}\right) \\
& +\alpha_{2}\left(\boldsymbol{M}_{i j}^{2} \boldsymbol{M}_{k l}^{2}+\boldsymbol{M}_{i k}^{2} \boldsymbol{M}_{j l}^{2}+\boldsymbol{M}_{i l}^{2} \boldsymbol{M}_{j k}^{2}\right) \\
& +\alpha_{3}\left(\boldsymbol{\delta}_{i j} \boldsymbol{M}_{k l}+\boldsymbol{\delta}_{i k} \boldsymbol{M}_{j l}+\boldsymbol{\delta}_{i l} \boldsymbol{M}_{j k}+\boldsymbol{M}_{i j} \boldsymbol{\delta}_{k l}+\boldsymbol{M}_{i k} \boldsymbol{\delta}_{j l}\right. \\
& \left.+\boldsymbol{M}_{i l} \boldsymbol{\delta}_{j k}\right)+\alpha_{4}\left(\boldsymbol{\delta}_{i j} \boldsymbol{M}_{k l}^{2}+\boldsymbol{\delta}_{i k} \boldsymbol{M}_{j l}^{2}+\boldsymbol{\delta}_{i l} \boldsymbol{M}_{j k}^{2}+\boldsymbol{M}_{i j}^{2} \boldsymbol{\delta}_{k l}\right.
\end{aligned}
$$




$$
\begin{aligned}
& \left.+\boldsymbol{M}_{i k}^{2} \boldsymbol{\delta}_{j l}+\boldsymbol{M}_{i l}^{2} \boldsymbol{\delta}_{j k}\right)+\alpha_{5}\left(\boldsymbol{M}_{i j} \boldsymbol{M}_{k l}^{2}+\boldsymbol{M}_{i k} \boldsymbol{M}_{j l}^{2}\right. \\
& \left.+\boldsymbol{M}_{i l} \boldsymbol{M}_{j k}^{2}+\boldsymbol{M}_{i j}^{2} \boldsymbol{M}_{k l}+\boldsymbol{M}_{i k}^{2} \boldsymbol{M}_{j l}+\boldsymbol{M}_{i l}^{2} \boldsymbol{M}_{j k}\right)
\end{aligned}
$$

where $\alpha_{i}=\alpha_{i}(s, v, e, \boldsymbol{M})$, are isotropic in $\boldsymbol{M}$ and satisfy Onsager's relations as well as the other symmetries previously established; thus, they should be admissible. Beris and Edwards ([2], Eq. (9.1-2), p. 329) excluded a cross-coupling between flux of entropy and conformation gradient (and flux of conformation and gradient of entropy) because this coupling is not allowed by the Curie's Principle ([93], p. 2476); however, later they stated that they excluded from the dissipation bracket some terms which were unlikely to contribute to the physics of the situation even though such terms were compatible with the Curie's Principle [119]; cross-coupling terms between different rank tensors are included, for example, in the description of stress-induced diffusion in Eqs. (9.2-6)-(9.2.8) of [2].

\subsection{The stress dyadic}

The term $\nabla v: \boldsymbol{L}^{\boldsymbol{v} v}: \nabla \boldsymbol{v}$ must also give a positive entropy production; therefore, $\boldsymbol{L}^{v v}$ is a positive definite fourth-rank tensor, the viscosity tetradic, and the viscous stress is $\boldsymbol{\tau}=$ $\boldsymbol{L}^{v v}: \nabla \boldsymbol{v}$. If Onsager's relations hold, then $\boldsymbol{L}_{i j k l}^{v v}=\boldsymbol{L}_{k l i j}^{v v}$. If the stress tensor is symmetric, as it commonly is in polymeric liquids, then $\boldsymbol{L}^{v v}$ has the following symmetries: $\boldsymbol{L}_{i j k l}^{v v}=\boldsymbol{L}_{j i k l}^{v v}$, and $\boldsymbol{L}_{i j k l}^{v v}=\boldsymbol{L}_{i j l k}^{v v}$ and the viscous stress is $\boldsymbol{\tau}=\boldsymbol{L}^{\boldsymbol{v} v}: \boldsymbol{D}$, where $\boldsymbol{D} \equiv\left(\nabla \boldsymbol{v}+\nabla \boldsymbol{v}^{\mathrm{T}}\right) / 2$ is the rate of strain.

The contribution to the entropy production rate (Eq. (95))

$$
\left(\sigma-\tilde{\pi} \boldsymbol{I}-\epsilon \boldsymbol{R}^{e v}-\boldsymbol{S}: \boldsymbol{R}^{\boldsymbol{M} v}\right): \nabla \boldsymbol{v}
$$

changes sign if the velocity is suddenly reversed [3]; therefore, it cannot be positive definite, and it should vanish. It can be split into two independent parts by defining the tensors

$$
\boldsymbol{R}_{i j k l}^{\boldsymbol{M D}} \equiv \frac{1}{2}\left(\boldsymbol{R}_{i j k l}^{\boldsymbol{M v}}+\boldsymbol{R}_{i j l k}^{\boldsymbol{M v}}\right)
$$

$\boldsymbol{R}_{i j k l}^{\boldsymbol{M W}} \equiv \frac{1}{2}\left(\boldsymbol{R}_{i j k l}^{\boldsymbol{M v}}-\boldsymbol{R}_{i j l k}^{\boldsymbol{M v}}\right)$

such that $\boldsymbol{R}^{M v}=\boldsymbol{R}^{M D}+\boldsymbol{R}^{M W}$. $\boldsymbol{R}^{M D}$ is symmetric with respect to transposition of the last two indices, whereas $\boldsymbol{R}^{M W}$ is skew symmetric with respect to transposition of the last two indices. Then

$$
\boldsymbol{R}^{M v}: \nabla v=R^{M D}: D+R^{M W}: W
$$

where $\boldsymbol{D}$ is the rate of strain and $\boldsymbol{W}$ is the vorticity dyadic. In polymeric liquids the stress dyadic is symmetric; therefore, $\sigma: \boldsymbol{W}=0 . \boldsymbol{R}^{e v}$ is an isotropic function of the symmetric tensor $\boldsymbol{M}$; therefore, it is symmetric and $\boldsymbol{R}^{e v}: \boldsymbol{W}=0$. Eq. (98) can be rewritten as

$$
\left(\sigma-\tilde{\pi} \boldsymbol{I}-\epsilon \boldsymbol{R}^{e v}-S: \boldsymbol{R}^{M D}\right): D+S: \boldsymbol{R}^{M W}: \boldsymbol{W}=0
$$

for any value of $\boldsymbol{D}$ and $\boldsymbol{W}$. The rate of strain and the vorticity are independent; therefore, the elastic stress $\sigma$ must obey the equation

$\boldsymbol{\sigma}=\tilde{\pi} \boldsymbol{I}+\epsilon \boldsymbol{R}^{e v}+\boldsymbol{S}: \boldsymbol{R}^{M D}$

which shows that the elastic stress is related to the part of the generation terms of entanglements and conformation that depend on the rate of strain - an equivalent relationship was derived by Öttinger [107]. It is important to point out that the expression of the elastic stress is derived here, as in the Generalized Bracket, Matrix, and GENERIC approaches, whereas it is postulated in the approach of Leonov. Eq. (102) also implies that

$S: R^{M W}: W=0$

for any skew symmetric tensor $\boldsymbol{W}$. However, Eq. (104) is not an additional constraint on $\boldsymbol{R}^{\boldsymbol{M} \boldsymbol{W}}$ because any fourth-rank tensor isotropic in $\boldsymbol{M}$, symmetric with respect to transposition of the first pair of indices and skew symmetric with respect to transposition of the second pair of indices enjoys that property [60].

The mechanical pressure $p$ (mean normal stress) is related to the thermodynamic pressure $-\tilde{\pi}$ by

$$
\begin{aligned}
p \equiv & -\frac{1}{3} \operatorname{tr} \boldsymbol{T}=-\tilde{\pi}-\frac{1}{3} \epsilon \operatorname{tr} \boldsymbol{R}^{e v}-\frac{1}{3} \boldsymbol{S}: \boldsymbol{R}^{\boldsymbol{M D}}: \boldsymbol{I} \\
& -\frac{1}{3} \boldsymbol{I}: \boldsymbol{L}^{\boldsymbol{v} v}: \boldsymbol{D} .
\end{aligned}
$$

To recover the definition of thermodynamic pressure at equilibrium with respect to transport $(\nabla \boldsymbol{v} \equiv \mathbf{0})$ and microstructural changes $\left(\boldsymbol{M} \equiv \boldsymbol{M}^{0}, e \equiv e^{0}\right)$, the relationship

$$
\begin{aligned}
& \epsilon\left(e^{0}, \boldsymbol{M}^{0}\right) \operatorname{tr} \boldsymbol{R}^{e v}\left(e^{0}, \boldsymbol{M}^{0}\right) \\
& \quad+\boldsymbol{S}\left(e^{0}, \boldsymbol{M}^{0}\right): \boldsymbol{R}^{\boldsymbol{M D}}\left(e^{0}, \boldsymbol{M}^{0}\right): \boldsymbol{I}=0
\end{aligned}
$$

should hold.

\subsection{Microstructure relaxation}

The last term in Eq. (95) must be non-negative because it is independent of the other terms in the expression of the entropy production rate. This term represents entropy generation due to re-equilibration of microstructure, i.e., relaxation and re-entanglement of the polymer molecules:

$-\left(\epsilon_{\mathrm{g}}^{0}+\boldsymbol{S}: \boldsymbol{M}_{\mathrm{g}}^{0}\right) \geq 0$.

If the dissipative processes leading to the relaxation of the polymer molecules and their re-entanglement are taken to be independent, then each of them must produce entropy:

$-\epsilon_{\mathrm{g}}^{0} \geq 0$

$-\boldsymbol{S}: \boldsymbol{M}_{\mathrm{g}}^{0} \geq 0$.

Not enough is known of the mechanisms of entanglement formation and destruction during flow and relaxation; therefore, it is preferable to use inequality 107 to check the 
thermodynamic consistency of constitutive equations which include explicitly the effect of changing entanglement concentration. Appendices B and C show that the expression of the entropy production rate and the transport equations of conformation hold both in inertial frames and rigid rotating frames.

\subsection{Specification of models}

Constitutive functions for the internal energy, the phenomenological coefficients, and the generation terms must be specified to close the equations of the model and thus permit theoretical predictions of flow and transport in polymeric liquids. This is in contrast to the case of isotropic unstructured liquids, where the thermal conductivity, the shear and bulk viscosities, and the internal energy function suffice to specify completely the model. The complete set of equations that describes flow and transport in a polymeric liquid is the following. The balance equations are

$$
\begin{aligned}
\dot{v}= & \nu \nabla \cdot \boldsymbol{v} \\
\rho \dot{\boldsymbol{v}}= & \nabla \cdot \boldsymbol{T}-\nabla \Theta \\
\rho \dot{u}= & -\nabla \cdot \boldsymbol{J}_{\mathrm{u}}+\boldsymbol{T}: \boldsymbol{D} \\
\rho \dot{\boldsymbol{s}}= & -\nabla \cdot \boldsymbol{J}_{\mathrm{s}}+\frac{1}{T}\left(\boldsymbol{\tau}: \boldsymbol{D}-\nabla T \cdot \boldsymbol{J}_{\mathrm{s}}-\nabla \epsilon \cdot \boldsymbol{J}_{\mathrm{e}}\right. \\
& \left.-\nabla \boldsymbol{S} \bullet \boldsymbol{J}_{\boldsymbol{M}}\right)+\frac{1}{T}\left(-\epsilon_{\mathrm{g}}^{0}-\boldsymbol{S}: \boldsymbol{M}_{\mathrm{g}}^{0}\right)
\end{aligned}
$$

$$
\begin{aligned}
& \rho \dot{e}=-\nabla \cdot \boldsymbol{J}_{\mathrm{e}}+\boldsymbol{R}^{e v}: \boldsymbol{D}+e_{\mathrm{g}}^{0} \\
& \rho \dot{\boldsymbol{M}}=-\nabla \cdot \boldsymbol{J}_{\boldsymbol{M}}+\boldsymbol{R}^{\boldsymbol{M} \boldsymbol{D}}: \boldsymbol{D}+\boldsymbol{R}^{\boldsymbol{M W}}: \boldsymbol{W}+\boldsymbol{M}_{\mathrm{g}}^{0} .
\end{aligned}
$$

The relationships between fluxes and linear phenomenological laws are

$$
\begin{aligned}
& \boldsymbol{T}=\boldsymbol{T}^{\mathrm{T}}=\boldsymbol{\sigma}+\boldsymbol{\tau} \\
& \boldsymbol{\sigma}=\boldsymbol{\sigma}^{\mathrm{T}}=\tilde{\pi} \boldsymbol{I}+\epsilon \boldsymbol{R}^{e v}+\boldsymbol{S}: \boldsymbol{R}^{\boldsymbol{M} \boldsymbol{D}} \\
& \boldsymbol{\tau}=\boldsymbol{\tau}^{\mathrm{T}}=\boldsymbol{L}^{v \boldsymbol{v}}: \boldsymbol{D} \\
& \boldsymbol{J}_{\mathrm{u}}=T \boldsymbol{J}_{\mathrm{s}}+\epsilon \boldsymbol{J}_{\mathrm{e}}+\boldsymbol{J}_{\boldsymbol{M}}: \boldsymbol{S} \\
& \boldsymbol{J}_{\mathrm{s}} \equiv-\boldsymbol{L}^{s s} \cdot \nabla T-\boldsymbol{L}^{s e} \cdot \nabla \epsilon-\boldsymbol{L}^{s \boldsymbol{M}} \bullet \nabla \boldsymbol{S} \\
& \boldsymbol{J}_{\mathrm{e}} \equiv-\boldsymbol{L}^{e s} \cdot \nabla T-\boldsymbol{L}^{e e} \cdot \nabla \epsilon-\boldsymbol{L}^{e \boldsymbol{M}} \bullet \nabla \boldsymbol{S} \\
& \boldsymbol{J}_{\boldsymbol{M}} \equiv-\boldsymbol{L}^{\boldsymbol{M} s} \cdot \nabla T-\boldsymbol{L}^{\boldsymbol{M} e} \cdot \nabla \epsilon-\boldsymbol{L}^{\boldsymbol{M} \boldsymbol{M}} \bullet \nabla \boldsymbol{S},
\end{aligned}
$$

\begin{tabular}{|c|c|c|c|}
\hline Physical quantity & Tensorial rank & Symmetries & Other properties \\
\hline$u$ & 0 & - & - \\
\hline$L^{s s}$ & 2 & $\boldsymbol{L}_{i j}^{s s}=\boldsymbol{L}_{j i}^{s s}$ & $\boldsymbol{a} \boldsymbol{a}: \boldsymbol{L}^{s s} \geq 0 \forall \boldsymbol{a}$ \\
\hline$L^{s e}$ & 2 & $\boldsymbol{L}_{i j}^{s e}=\boldsymbol{L}_{j i}^{s e}$ & $\boldsymbol{L}^{s e}=\boldsymbol{L}^{e s}$ \\
\hline$L^{e s}$ & 2 & $\boldsymbol{L}_{i j}^{e s}=\boldsymbol{L}_{j i}^{e s}$ & $\boldsymbol{L}^{e s}=\boldsymbol{L}^{s e}$ \\
\hline$L^{e e}$ & 2 & $\boldsymbol{L}_{i j}^{e e}=\boldsymbol{L}_{j i}^{e e}$ & $\boldsymbol{a} \boldsymbol{a}: \boldsymbol{L}^{e e} \geq 0 \quad \forall \boldsymbol{a}$ \\
\hline$L^{s M}$ & 4 & $\boldsymbol{L}_{i j k l}^{s M}=\boldsymbol{L}_{i j l k}^{s M}$ & $\boldsymbol{L}_{i j k l}^{s \boldsymbol{M}}=\boldsymbol{L}_{l i j k}^{\boldsymbol{M} s}$ \\
\hline$L^{M s}$ & 4 & $\boldsymbol{L}_{i j k l}^{\boldsymbol{M} s}=\boldsymbol{L}_{i k j l}^{\boldsymbol{M} s}$ & $\boldsymbol{L}_{i j k l}^{\boldsymbol{M} s}=\boldsymbol{L}_{l i j k}^{s \boldsymbol{M}}$ \\
\hline$L^{e M}$ & 4 & $\boldsymbol{L}_{i j k l}^{e \boldsymbol{M}}=\boldsymbol{L}_{i j l k}^{e \boldsymbol{M}}$ & $\boldsymbol{L}_{i j k l}^{e \boldsymbol{M}}=\boldsymbol{L}_{l i j k}^{\boldsymbol{M} e}$ \\
\hline$L^{M e}$ & 4 & $\boldsymbol{L}_{i j k l}^{\boldsymbol{M} e}=\boldsymbol{L}_{i k j l}^{\boldsymbol{M} e}$ & $\boldsymbol{L}_{i j k l}^{\boldsymbol{M} e}=\boldsymbol{L}_{l i j k}^{e \boldsymbol{M}}$ \\
\hline$L^{M M}$ & 6 & $\boldsymbol{L}_{i j k l p q}^{M M}=\boldsymbol{L}_{i k j l p q}^{M M}=\boldsymbol{L}_{i j k l q p}^{M M}=\boldsymbol{L}_{l p q i j k}^{M M}$ & $a b c \bullet L^{M M} \bullet a b c \geq 0 \forall a, b, c$ \\
\hline$L^{v v}$ & 4 & $\boldsymbol{L}_{i j k l}^{v v}=\boldsymbol{L}_{j i k l}^{v v}=\boldsymbol{L}_{i j l k}^{v v}=\boldsymbol{L}_{k l i j}^{v v}$ & $a b: L^{v v}: a b \geq 0 \quad \forall a, b$ \\
\hline $\boldsymbol{R}^{e v}$ & 2 & $\boldsymbol{R}_{i j}^{e v}=\boldsymbol{R}_{j i}^{e v}$ & \\
\hline$R^{M D}$ & 4 & $\boldsymbol{R}_{i j k l}^{M \boldsymbol{D}}=\boldsymbol{R}_{j i k l}^{M \boldsymbol{D}}=\boldsymbol{R}_{i j l k}^{M \boldsymbol{D}}$ & \\
\hline$R^{M W}$ & 4 & $\boldsymbol{R}_{i j k l}^{M W}=\boldsymbol{R}_{j i k l}^{M W}=-\boldsymbol{R}_{i j l k}^{M W}$ & $S: \boldsymbol{R}^{M W}: \boldsymbol{W}=0$ \\
\hline$e_{\mathrm{g}}^{0}$ & 0 & - & $\epsilon e_{\mathrm{g}}^{0} \leq 0$ \\
\hline $\boldsymbol{M}_{\mathrm{g}}^{0}$ & 2 & $\boldsymbol{M}_{g i j}^{0}=\boldsymbol{M}_{g j i}^{0}$ & $\boldsymbol{S}: \boldsymbol{M}_{\mathrm{g}}^{0} \leq 0$ \\
\hline
\end{tabular}

and the definitions of temperature, thermodynamic pressure, and other conjugate variables are

$$
\begin{aligned}
T & \equiv \frac{\partial u}{\partial s} \\
\tilde{\pi} & \equiv \frac{\partial u}{\partial v} \\
\epsilon & \equiv \frac{\partial u}{\partial e} \\
\boldsymbol{S} & \equiv \frac{\partial u}{\partial \boldsymbol{M}} .
\end{aligned}
$$

Table 1

Phenomenological coefficients and material functions that must be specified through constitutive laws to close the set of balance equations of mass, momentum, internal energy, entropy, entanglement, and conformation (Eqs. (110)-(115)) together with the relationships between the diffusive fluxes and affinities (Eqs. (116)-(122)), and restrictions placed on the constitutive laws. The independent variables are $s, v, \boldsymbol{M}, e$. All the tensors are isotropic functions of $\boldsymbol{M}$ 
The phenomenological coefficients and the material functions that must be specified through constitutive laws are listed in Table 1, and the restrictions on the constitutive laws are summarized there.

Kinetic theories play a very important role in selecting appropriate constitutive functions by suggesting particular dependencies of the free energy on the state variables and particular forms of the diffusive and generation terms in the transport equations $[12,120,27,121]$. However, the transport and constitutive equations derived from kinetic theories rely on specific micromechanical models of microstructure and should be used to complement, not substitute, macroscopic theories.

\section{Simplified models}

In practical applications it is often possible to neglect some of the phenomena included in Eqs. (110)-(122), and therefore to simplify the modeling. For example, polymer melts and solutions behave as incompressible liquids in most processing flows. Diffusion and heat transfer seem usually negligible in fast processes like coating flows, and they are commonly neglected in viscoelastic flow modeling ([122] and references therein). The most relevant reduced equation sets that apply in the simpler situations of incompressible flow, non-diffusing flows, and isothermal flows are reported in the next sections; other interesting cases are summarized in Appendices D and E.

\subsection{Incompressible liquid, non-diffusing polymer conformation and entanglements}

The equation set describing the flow of an incompressible, non-diffusing polymer solution or melt is obtained from Eqs. (67)-(77) by setting $v$ constant and by zeroing the diffusive fluxes of entanglements $\boldsymbol{J}_{\mathrm{e}}$ and conformation $\boldsymbol{J}_{\boldsymbol{M}}$ :

$$
\begin{aligned}
& 0=\nabla \cdot \boldsymbol{v} \\
& \rho \dot{u}=-\nabla \cdot \boldsymbol{J}_{\mathrm{u}}+\boldsymbol{T}: \boldsymbol{D} \\
& \rho \dot{s}=-\nabla \cdot \boldsymbol{J}_{\mathrm{s}}+\frac{1}{T}\left(-\boldsymbol{J}_{\mathrm{s}} \cdot \nabla T+\boldsymbol{\tau}: \boldsymbol{D}-\epsilon e_{\mathrm{g}}^{0}-\boldsymbol{S}: \boldsymbol{M}_{\mathrm{g}}^{0}\right) \\
& \rho \dot{\boldsymbol{e}}=\boldsymbol{R}^{e \boldsymbol{v}}: \boldsymbol{D}+e_{\mathrm{g}}^{0} \\
& \rho \dot{\boldsymbol{M}}=\boldsymbol{R}^{\boldsymbol{M D}}: \boldsymbol{D}+\boldsymbol{R}^{\boldsymbol{M} \boldsymbol{W}}: \boldsymbol{W}+\boldsymbol{M}_{\mathrm{g}}^{0} \\
& \boldsymbol{T}=\boldsymbol{\sigma}+\boldsymbol{\tau} \\
& \boldsymbol{\sigma}=-\pi \boldsymbol{I}+\epsilon \boldsymbol{R}^{e v}+\boldsymbol{S}: \boldsymbol{R}^{\boldsymbol{M D}} \\
& \boldsymbol{\tau}=\boldsymbol{L}^{\boldsymbol{v} \boldsymbol{v}}: \boldsymbol{D} \\
& \boldsymbol{J}_{\mathrm{u}}=T \boldsymbol{J}_{\mathrm{s}} \\
& \boldsymbol{J}_{\mathrm{s}}=-\boldsymbol{L}^{s s} \cdot \nabla T .
\end{aligned}
$$

Constitutive equations must be specified for the internal energy $u(s, \boldsymbol{M}, e)$, the viscosity tetradic $\boldsymbol{L}^{\boldsymbol{v} v}(s, \boldsymbol{M}, e)$, the coupling between rate of strain and entanglement generation $\boldsymbol{R}^{e v}(s, \boldsymbol{M}, e)$, the coupling between rate of strain and conformation generation $\boldsymbol{R}^{\boldsymbol{M} D}(s, \boldsymbol{M}, e)$, the thermal conductivity tensor $\boldsymbol{L}^{s s}(s, \boldsymbol{M}, e)$, and the relaxation terms $e_{\mathrm{g}}^{0}$ and $\boldsymbol{M}_{\mathrm{g}}^{0}$; the coupling between vorticity and conformation generation $\boldsymbol{R}^{\boldsymbol{M} \boldsymbol{W}}(s, \boldsymbol{M}, e)$ is given by Eq. (C.2).

\subsection{Isothermal, incompressible, non-diffusing flows}

Isothermal, incompressible flows are the most important class of process flows of polymer solutions. Virtually all coating flows and many polymer processing applications fall into this category. The balance Eqs. (78)-(82) become

$$
\begin{aligned}
& 0=\nabla \cdot \boldsymbol{v} \\
& \rho \dot{\boldsymbol{v}}=\nabla \cdot \boldsymbol{T}-\nabla \Theta \\
& \rho \dot{u}=-\nabla \cdot \boldsymbol{J}_{\mathrm{u}}+\boldsymbol{T}: \nabla \boldsymbol{v} \\
& \rho \dot{\boldsymbol{s}}=-\nabla \cdot \boldsymbol{J}_{\mathrm{s}}+s_{\mathrm{g}} \\
& \rho \dot{\boldsymbol{e}}=e_{\mathrm{g}} \\
& \rho \dot{\boldsymbol{M}}=\boldsymbol{M}_{\mathrm{g}} .
\end{aligned}
$$

Because the temperature is constant, it is convenient to use the Helmholtz free energy $a \equiv u-T s \equiv a=a(T, e, \boldsymbol{M})$ rather than the internal energy to describe these flows; thus

$\dot{a}=\dot{u}-\dot{T} s-T \dot{s}=-s \dot{T}+\epsilon \dot{e}+S: \dot{M} ;$

therefore

$$
\frac{\partial a}{\partial T}=s, \quad \frac{\partial a}{\partial e}=\epsilon, \quad \frac{\partial a}{\partial \boldsymbol{M}}=\boldsymbol{S} .
$$

By using Eqs. (139)-(143), the local entropy production inequality can be put in the form

$$
\begin{aligned}
T \dot{S}_{\mathrm{g}}= & \nabla \cdot\left(-\boldsymbol{J}_{\mathrm{u}}+T \boldsymbol{J}_{\mathrm{s}}\right)-\boldsymbol{J}_{\mathrm{s}} \cdot \nabla T+\boldsymbol{T}: \boldsymbol{D} \\
& -\boldsymbol{M}_{\mathrm{g}}: \boldsymbol{S}-e_{\mathrm{g}} \epsilon \geq 0 .
\end{aligned}
$$

Because the flow is isothermal, $\dot{T}=0$ and $\nabla T=0$, the fluxes of internal energy and entropy are constitutively undetermined and behave as Lagrange multipliers to ensure that the balance equations of internal energy and entropy are always satisfied. The relationship $\boldsymbol{J}_{\mathrm{u}}=T \boldsymbol{J}_{\mathrm{s}}$ must still hold. The balance laws of internal energy and entropy need not be considered further because internal energy, entropy, and their diffusive fluxes do not enter the balance equations of mass, momentum, conformation and entanglements.

The stress tensor $\boldsymbol{T}$ is also constitutively determined only up to an isotropic value, because the velocity field of an incompressible material is divergence-free (Eq. (137)): 


$$
\begin{aligned}
(\boldsymbol{T}+\pi \boldsymbol{I}): \boldsymbol{D} & =\boldsymbol{T}: \boldsymbol{D}+\pi \boldsymbol{I}: \boldsymbol{D} \\
& =\boldsymbol{T}: \boldsymbol{D}+\pi \nabla \cdot \boldsymbol{v}=\boldsymbol{T}: \boldsymbol{D} .
\end{aligned}
$$

The field $\pi(\boldsymbol{x})$ is determined by solving the balance equations together with boundary conditions, and the stress tensor follows the equation

$\boldsymbol{T}=-\pi \boldsymbol{I}+\boldsymbol{\sigma}+\boldsymbol{\tau}$

where $\sigma$ is the elastic stress and $\boldsymbol{\tau}$ is the viscous stress. The local entropy production inequality simplifies therefore to

$\boldsymbol{\sigma}: \boldsymbol{D}+\boldsymbol{\tau}: \boldsymbol{D}-\boldsymbol{M}_{\mathrm{g}}: \boldsymbol{S}-e_{\mathrm{g}} \epsilon \geq 0$.

If the rates of generation of conformation $\boldsymbol{M}_{\mathrm{g}}$ and entanglements $e_{\mathrm{g}}$ depend linearly on the velocity gradient $\nabla \boldsymbol{v}$, then the analysis in Section 5 holds and thus

$e_{\mathrm{g}}=e_{\mathrm{g}}^{0}+\boldsymbol{R}^{e v}: \boldsymbol{D}$

$M_{\mathrm{g}}=M_{\mathrm{g}}^{0}+R^{M D}: D+R^{M W}: W$,

and

$\sigma=\epsilon \boldsymbol{R}^{e v}+S: R^{M D}$

$\tau=L^{v v}: D$

$0=S: R^{M W}: W$.

In fully non-linear theories (theories in which the generation terms may depend non-linearly on the rate of strain), Eq. (148) should be used to check constitutive assumptions against the second law of thermodynamics, as discussed in Section 8.

\subsection{Isothermal, incompressible, non-diffusing flows, unentangled polymer}

As in the preceeding section, the energy and entropy equations decouple from the mass, momentum, and conformation balance equations; thus, the relevant balance equations are

$0=\nabla \cdot \boldsymbol{v}$

$\rho \dot{\boldsymbol{v}}=\nabla \cdot \boldsymbol{T}-\nabla \Theta$

$\rho \dot{\boldsymbol{M}}=\boldsymbol{M}_{\mathrm{g}}$,

and the local entropy production inequality is

$\boldsymbol{\sigma}: \boldsymbol{D}+\boldsymbol{\tau}: \boldsymbol{D}-\boldsymbol{M}_{\mathrm{g}}: S \geq 0$,

with $a=a(\boldsymbol{M})$.

Assuming a linear coupling between generation of conformation $\boldsymbol{M}_{\mathrm{g}}$ and velocity gradient $\nabla \boldsymbol{v}$ (as in Section 5)

$\boldsymbol{M}_{\mathrm{g}}=\boldsymbol{M}_{\mathrm{g}}^{0}+\boldsymbol{R}^{M D}: D+\boldsymbol{R}^{M W}: \boldsymbol{W}$,

then

$\sigma=S: R^{M D}$

$\tau=L^{v v}: D$
$0=S: R^{M W}: W$

In non-linear theories, Eq. (157) should be used to check constitutive assumptions against the second law of thermodynamics. Eqs. (154)-(156), (158)-(161)) coincide with Eqs. (8.1-7a)-(8.1-7c) of Beris and Edwards [2] and with the equations proposed by Jongschaap et al. [3] by identifying

$$
\begin{gathered}
\underbrace{\boldsymbol{R}_{i j k l}^{\boldsymbol{M D}}=}_{\text {this work }} \delta_{i k} M_{j l}+\delta_{i l} M_{j k}+\delta_{j k} M_{i l}+\delta_{j l} M_{i k} \\
+\underbrace{\boldsymbol{L}_{i j k l}}_{\text {Generalized Bracket }}=\underbrace{\boldsymbol{\Lambda}_{i j k l}^{1}}_{\text {Matrix Model }}
\end{gathered}
$$

where $\boldsymbol{L}_{i j k l}$ is the tensor defined in Eq. (41) in Section 3.2 (Eq. (8.1-7c) of [2]), $\boldsymbol{\Lambda}^{1}$ is the tensor defined in Eq. (58) (Eq. (36) of [3]); and by identifying the viscosity tensors $\boldsymbol{L}^{\boldsymbol{v} v} \equiv \boldsymbol{Q}=\boldsymbol{\eta}$. This shows that the simple thermodynamic approach yields the same equations as the Generalized Bracket and Matrix Model, at least when the equations of change of microstructure depend linearly on the velocity gradient and diffusion is neglected. The model of Leonov is not recovered because in that model the stress is postulated rather than derived - see the discussion in Section 5.3 above.

\section{Evolution equations of conformation and entanglement concentration}

The evolution equations of conformation and entanglement concentration must reflect the effects of local macroscopic deformation on the material and its internal relaxations. Eqs. (114) and (115) can be simplified substantially by using micromechanical models of polymer behavior.

The generation terms $\boldsymbol{M}_{\mathrm{g}}$ and $e_{\mathrm{g}}$ represent changes due to internal processes only, i.e., processes that do not involve transfer through the boundary of a liquid element. The discussion in the following subsections is therefore restricted to internal processes. A simpler form of the generation terms of conformation $\boldsymbol{M}_{\mathrm{g}}$ is presented below in Section 7.2, where the effect of the macroscopic deformation gradient on coil stretching, coil rotation with respect to neighboring coils, and collective rotation of coils splits into independent parts. The resulting equation of change of conformation is a particular case of Eq. (115) and includes the mechanisms of affine deformation, partial slip as put forward by Gordon and Schowalter [123,124], incomplete retraction [106,125], and complete retraction [11,96], which are at the basis of all known rate-type constitutive equations for dilute solutions, semidilute unentangled and entangled solutions, concentrated solutions, and melts.

No general mechanism of entanglement formation and destruction is known: published models of polymer behavior that account explicitly for an entanglement variable are reviewed by Yosick et al. [126]. The salient difference between the models that have been proposed so far is whether 
or not the velocity gradient affects the rates at which entanglements break and reform.

The generation terms of entanglements and conformation are related respectively to the rates of change due to internal processes of the zeroth and second moment of the segment distribution function $\Psi(\boldsymbol{r}, \boldsymbol{x}, t)$. If only internal processes are considered, the distribution function obeys the equation $[12,39,127]$

$$
\frac{\partial \Psi}{\partial t}+\boldsymbol{v} \cdot \nabla \Psi+\frac{\partial}{\partial \boldsymbol{r}} \cdot \Psi \dot{\boldsymbol{r}}=\Psi_{\mathrm{p}}-\Psi_{\mathrm{d}},
$$

where $\Psi_{\mathrm{p}}(\boldsymbol{r}) \mathrm{d} \boldsymbol{r}$ and $\Psi_{\mathrm{d}}(\boldsymbol{r}) \mathrm{d} \boldsymbol{r}$ are respectively the rates of production and destruction of segments of length $\boldsymbol{r}$ per unit mass. In theories of unentangled solutions, segments represent the end-to-end connectors of polymer molecules; therefore, $\Psi_{\mathrm{p}}(\boldsymbol{r}) \mathrm{d} \boldsymbol{r} \equiv \Psi_{\mathrm{d}}(\boldsymbol{r}) \mathrm{d} \boldsymbol{r} \equiv 0$ if no polymerization reactions occur. In theories of entangled solutions, segments portray the connectors between successive entanglements on the same polymer chain; therefore, $\Psi_{\mathrm{p}}(\boldsymbol{r}) \mathrm{d} \boldsymbol{r}$ and $\Psi_{\mathrm{d}}(\boldsymbol{r}) \mathrm{d} \boldsymbol{r}$ need not vanish.

Taking the zeroth moment of Eq. (163), recalling that $\int_{\boldsymbol{r} \in R^{3}} \mathrm{~d} \boldsymbol{r} \Psi=c_{\mathrm{p}}$, and applying the divergence theorem leads to

$\dot{c}_{\mathrm{p}}=\int_{\boldsymbol{r} \in R^{3}} \mathrm{~d} \boldsymbol{r} \Psi_{\mathrm{p}}-\int_{\boldsymbol{r} \in R^{3}} \mathrm{~d} \boldsymbol{r} \Psi_{\mathrm{d}}$

irrespective of the expression of $\dot{\boldsymbol{r}}$. In an unentangled, nonreacting solution, Eq. (164) simply states that the number of segments per unit mass does not change due to internal processes. In an entangled solution of linear polymers the number of segments (defined as connectors between two entanglements or between an entanglement and a loose end) per unit mass is $c_{\mathrm{p}}=c_{\mathrm{m}}+2 e$, where $e$ is the number of entanglements per unit mass and $c_{\mathrm{m}}$ is the number of polymer molecules per unit mass. If no chemical reactions occur, $c_{\mathrm{m}}$ is constant and the number of segments per unit mass can change due to breaking and forming of entanglements, $\dot{c}_{\mathrm{p}}=2 \dot{e}$.

The rate of change of the second moment of $\Psi$ depends on the expression of $\dot{r}$. The conformation of a segment is changed by effects of flow and deformation, and by effects of Brownian motion and entropic elasticity. Splitting these effects as

$\dot{\boldsymbol{r}} \equiv \boldsymbol{f}(\nabla \boldsymbol{v}, \boldsymbol{r})+\boldsymbol{g}(\boldsymbol{r}, \Psi)$

leads to

$$
\begin{aligned}
\dot{\boldsymbol{M}}= & v \boldsymbol{M}_{\mathrm{g}}=\int_{\boldsymbol{r} \in R^{3}} \mathrm{~d} \boldsymbol{r} \Psi(\boldsymbol{f} \boldsymbol{r}+\boldsymbol{r} \boldsymbol{f})+\int_{\boldsymbol{r} \in R^{3}} \mathrm{~d} \boldsymbol{r} \Psi(\boldsymbol{g} \boldsymbol{r}+\boldsymbol{r} \boldsymbol{g}) \\
& +\int_{\boldsymbol{r} \in R^{3}} \mathrm{~d} \boldsymbol{r} \Psi_{\mathrm{p}} \boldsymbol{r} \boldsymbol{r}-\int_{\boldsymbol{r} \in R^{3}} \mathrm{~d} \boldsymbol{r} \Psi_{\mathrm{d}} \boldsymbol{r} \boldsymbol{r} .
\end{aligned}
$$

The integrals in Eqs. (164) and (166) can be expressed in terms of $c_{\mathrm{p}}$ (or $e$ ) and $\boldsymbol{M}$ only if $\boldsymbol{f}(\nabla \boldsymbol{v}, \boldsymbol{r}), \boldsymbol{g}(\boldsymbol{r}, \Psi), \Psi_{\mathrm{p}}$, and $\Psi_{\mathrm{d}}$ have very simple forms. Yet, these integrals can be used to suggest expressions of the generation terms of conformation $\boldsymbol{M}_{\mathrm{g}}$ and entanglements $e_{\mathrm{g}}$, as described in Sections 7.1 and 7.2 .

\subsection{Rate of change of entanglements by internal processes}

The rate of generation of entanglements $e_{\mathrm{g}}$ is the rate of change of entanglement density $\rho e$ due to internal processes. It is related to the rate of change of segment density $\rho c_{\mathrm{p}}$. Because the number of polymer segments per unit mass equals the number of polymer molecules per unit mass plus twice the number of entanglements per unit mass, $c_{\mathrm{p}}=$ $c_{\mathrm{m}}+2 e$, and $c_{\mathrm{m}}$ is constant,

$\nu e_{\mathrm{g}}=\dot{e}=\frac{1}{2} \dot{c}_{\mathrm{p}}$.

Eqs. (164) and (167) give

$\nu e_{\mathrm{g}}=\frac{1}{2} \int_{\boldsymbol{r} \in R^{3}} \mathrm{~d} \boldsymbol{r} \Psi_{\mathrm{p}}-\frac{1}{2} \int_{\boldsymbol{r} \in R^{3}} \mathrm{~d} \boldsymbol{r} \Psi_{\mathrm{d}} \equiv e_{\mathrm{p}}-e_{\mathrm{d}}$

where the specific rate of entanglement generation has been split into a production and a destruction terms $e_{\mathrm{p}}, e_{\mathrm{d}} \geq 0$.

There is no consensus in the literature on the general expressions for $\Psi_{\mathrm{p}}$ and $\Psi_{\mathrm{d}}$. The simplest assumption about the rate of production of entanglement is that entanglement formation is a random process driven by thermal fluctuations and is independent of flow and coil deformation [128], whence $\Psi_{\mathrm{p}}=k \Psi^{0}$, where $k$ is a rate constant and $\Psi^{0}$ is the equilibrium distribution function of segments. Graessley [129] considered simple shear flow and argued that the rate of entanglement formation may depend on the contact time between neighboring polymer chains, i.e., the time in which the centers of mass of two chains are closer than a critical distance, approximately equal to the radius of gyration of the polymer coil. This contact time is inversely proportional to the relative velocity of molecules, identified by Graessley with the product of shear rate and characteristic molecular size; therefore, the rate of entanglement formation drops with growing shear rate, and the number of entanglements per polymer molecule is lower during flow than at equilibrium. Graessley argued that Lodge's assumption of first-order kinetics of entanglement formation is inadequate and that the rate of production of entanglements should be higher when few entanglements are present because the molecules are "much freer of encumbrances" ([129], p. 2700). To estimate the contact time between polymer molecules, Graessley treated the flowing coils as spheres whose radius corresponded to the molecules' equilibrium sizes; he neglected any flow-induced anisotropic shape. This approximation is reasonable at moderate rates of strain in weakly-stretching flows, such as simple shear flow; it becomes questionable in flows strong enough to distort considerably the polymer coils. The rate of production of entanglements may depend therefore on the segments' lengths and on their orientations with respect to the principal directions of the rate of strain. In general, the rate of production of segments may depend on the segment distribution function $\Psi$, segment stretch, segment density, local value of the velocity gradient, and the other thermodynamic variables ( $v$ and $T$ or $s$ ). 
Similar considerations apply to the rate of destruction of entanglements. Lodge [128] assumed that the rate of destruction of entanglements follows linear kinetics, $\Psi_{\mathrm{d}}=k \Psi$. Graessley [129] suggested that the rate of destruction of entanglements should rise with shear rate because the rate of separation of polymer coils grows with shear rate. Marrucci et al. [130] suggested that the rate of destruction of entanglements should depend on the elastic stress rather than the velocity gradient because structural rearrangements occur during relaxation processes, and they assumed that elastically stressed molecules disentangle faster. Of course, stressed molecules are extended and bent ones; therefore, the argument of Marrucci et al. [130] can be reformulated in terms of molecular conformation dyadic rather than elastic stress, i.e., in terms of polymer segment's stretch and orientation. The relationship between rate of destruction of entanglements and elastic stress was slightly modified in a later work [131]. Other expressions of the rate of destruction of entanglements involving the elastic stress were proposed by De Cleyn and Mewis [132] and by Mewis and Denn [127]. Liu et al. [133] postulated that the rate of destruction of entanglements rises with the rate of strain. A collection of published expressions of the rates of production and destruction of entanglements has been compiled by Yosick et al. ([126], Table 1), who also slightly modified the model of Liu et al. [133].

The concept of persistent straining seems also important in describing how polymer molecules entangle and disentangle. The strain is persistent when an ensemble of polymer coils rotates with the same angular velocity of the eigenvectors of the rate of strain [15]. If molecular conformation is included in a model, then the straining is persistent when the principal direction of extensional straining is aligned with the principal direction of elongation of the conformation tensor [63]; the liquid is recoiling when the principal direction of elongation of the conformation tensor is aligned with the principal direction of contraction of $\boldsymbol{D}$, and the principal direction of contraction of the conformation tensor is aligned with the principal direction of extension of the rate of strain; the straining is intermittent in other situations. Of course, in three-dimensional flows with a non-uniform velocity gradient, the liquid could be recoiling along one axis while suffering persistent or intermittent straining in the perpendicular plane.

From the point of view of a description in terms of local expectation values, it is reasonable to assume that the rates of production and destruction of entanglements may depend on the thermodynamic variables $T$ (or $s$ ), $\nu, e$, and $\boldsymbol{M}$, and the rate of strain - they must be independent of the vorticity $W$ ([60], for details)

$e_{\mathrm{p}}=e_{\mathrm{p}}(T, v, e, \boldsymbol{M}, \boldsymbol{D})$

$e_{\mathrm{d}}=e_{\mathrm{d}}(T, v, e, \boldsymbol{M}, \boldsymbol{D})$.

At equilibrium the number of entanglements per unit mass should be constant; therefore, the rates of entanglement production and destruction are related by
$e_{\mathrm{p}}\left(T, v, e^{0}, c_{\mathrm{p}} N l^{2} \boldsymbol{I}, \mathbf{0}\right)=e_{\mathrm{d}}\left(T, v, e^{0}, c_{\mathrm{p}} N l^{2} \boldsymbol{I}, \mathbf{0}\right)$

where $e^{0}$ and $c_{\mathrm{p}} N l^{2} \boldsymbol{I}$ are the equilibrium values of $e$ and $\boldsymbol{M}$. Hereafter the constitutive laws for $e_{\mathrm{p}}$ and $e_{\mathrm{d}}$ are not assumed to be linear in $\boldsymbol{D}$ (unlike in Section 5.1), and this has interesting implications for the formula of the elastic stress and for the local entropy production inequality, as discussed in Section 8.

\subsection{Rate of change of conformation by internal processes}

The generation term of conformation $\boldsymbol{M}_{\mathrm{g}}$ is the rate of change of conformation $\boldsymbol{M} \equiv c_{\mathrm{p}}\langle\boldsymbol{r} \boldsymbol{r}\rangle$ by internal processes. The average molecular conformation per unit mass $\boldsymbol{M}$ can change if the end-to-end distance of the segments $\boldsymbol{r}$ changes or if the number of segments per unit mass $c_{\mathrm{p}}$ is not constant. Polymer coils stretch and rotate by action of the velocity gradient. Entropic elasticity drives the coils towards a most-probable average end-to-end distance, $\bar{r} \equiv \sqrt{N l^{2}}$, where $N$ is the number of Kuhn steps and $l$ is the length of a Kuhn step ([7], pp. 411-412). When a segment represents the end-to-end connector of a polymer molecule (as in dilute and semi-dilute unentangled solutions and melts), then the number of segments per unit mass can change only if a polymerization reaction is occurring. When a segment portrays an entanglement-to-entanglement connector (as in semi-dilute and concentrated entangled solutions and melts), then the number of segments per unit mass can change due to breaking and forming of entanglements. Of course, this carries the tacit assumption that unentangled strands do not contribute to the stress.

It is convenient to split the rate of generation of conformation per unit mass $\nu \boldsymbol{M}_{\mathrm{g}}$ into three parts representing coils' stretch-and-orientation by action of flow and deformation, coils' relaxation by effect of entropic elasticity and Brownian motion, and change in coils' conformation due to formation and destruction of entanglements:

$$
\begin{aligned}
v \boldsymbol{M}_{\mathrm{g}}= & \underbrace{F(\nabla \boldsymbol{v}, \boldsymbol{M}, e)}_{\text {deformation }}+\underbrace{G(\boldsymbol{M}, e)}_{\text {relaxation }} \\
& +\underbrace{H(\nabla \boldsymbol{v}, \boldsymbol{M}, e)}_{\text {entanglement and disentanglement }}
\end{aligned}
$$

where the function $H(\nabla \boldsymbol{v}, \boldsymbol{M}, e)$ should be related to the rates of generation and destruction of entanglements. Comparing Eq. (172) to Eq. (166), and recalling that $\dot{\boldsymbol{M}}=v \boldsymbol{M}_{\mathrm{g}}$ when only internal processes are considered, leads to

$$
\begin{aligned}
& \mathcal{F}(\nabla \boldsymbol{v}, \boldsymbol{M}, e) \equiv \int_{\boldsymbol{r} \in R^{3}} \mathrm{~d} \boldsymbol{r} \Psi(\boldsymbol{f} \boldsymbol{r}+\boldsymbol{r} \boldsymbol{f}) \\
& \mathcal{G}(\boldsymbol{M}, e) \equiv \int_{\boldsymbol{r} \in R^{3}} \mathrm{~d} \boldsymbol{r} \Psi(\boldsymbol{g} \boldsymbol{r}+\boldsymbol{r} \boldsymbol{g}) \\
& \mathcal{H}(\nabla \boldsymbol{v}, \boldsymbol{M}, e) \equiv \int_{\boldsymbol{r} \in R^{3}} \mathrm{~d} \boldsymbol{r} \Psi_{\mathrm{p}} \boldsymbol{r} \boldsymbol{r}-\int_{\boldsymbol{r} \in R^{3}} \mathrm{~d} \boldsymbol{r} \Psi_{\mathrm{d}} \boldsymbol{r} \boldsymbol{r} .
\end{aligned}
$$




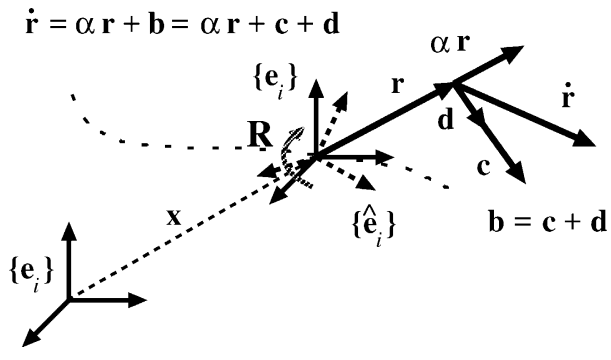

Fig. 3. Decomposition of the motion of a material filament into a stretch $\alpha \boldsymbol{r}$ and a rotation $\boldsymbol{b}$, the latter with respect to a chosen frame of reference $\left\{\boldsymbol{e}_{i}\right\} . \boldsymbol{c}$ is the rotation with respect to the frame $\left\{\hat{\boldsymbol{e}}_{i}\right\}$, which rotates with an angular velocity $\boldsymbol{R}$ with respect to $\left\{\boldsymbol{e}_{i}\right\}$; thus, $\boldsymbol{c}$ describes the orientation of filament $\boldsymbol{r}$ with respect to other filaments in an ensemble that rotates with angular velocity $\boldsymbol{R}$, whereas $\boldsymbol{d}$ describes rigid-body rotation of all the filaments of an ensemble.

\subsubsection{Conformation changes by flow and deformation}

Virtually all microscopic theories of polymer dynamics approximate a polymer segment with an infinitesimal linear element, or a sequence of connected infinitesimal linear elements $[12,41,11]$. The motion of an infinitesimal (on the macroscopic length scale of the flow) material filament $\ell$ is instructive. Up to first order in $|\ell|$, it is $\dot{\ell}=\ell \cdot \nabla \boldsymbol{v} \equiv$ $\boldsymbol{\ell} \cdot(\boldsymbol{D}+\boldsymbol{W})$ and can be broken into a stretch along the axis, a rotation with respect to the center of mass of the material element in which $\boldsymbol{\ell}$ is embedded and a solid body rotation with the material element (Fig. 3):

$$
\begin{aligned}
\dot{\ell}= & \underbrace{\frac{\ell \ell: D}{\ell \cdot \ell} \ell}_{\text {rate of stretching }}+\underbrace{\ell \cdot\left(D-\frac{\ell \ell: D}{\ell \cdot \ell} I\right)}_{\text {relative rotation rate }} \\
+\underbrace{\ell \cdot W}_{\text {solid-body rotation rate }} & .
\end{aligned}
$$

The vorticity dyadic $\boldsymbol{W}$ corresponds to the average angular velocity (with respect to a chosen frame of reference) of the material element only if the material element is isotropic [134] or, more generally, symmetric with respect to reflections about the principal axes of the rate-of-strain $\boldsymbol{D}$.

Polymer segments are finitely extensible unlike material filaments; therefore, the contribution of the stretch to their instantaneous incremental deformation should decrease with their extension. Also, bundles of stretched coils are not isotropic and can rotate at an average angular velocity different from half the vorticity, the angular velocity of isotropic liquid "particles". The difference between the average angular velocity of the coils and the vorticity can be related to the concept of a deformational part of the vorticity [135]; in fact, such a difference of angular velocities is independent of the choice of frame and so is objective.

If stretch, rotation, and relaxation are regarded as independent phenomena, then a more general time rate of change of polymer segments due to flow and deformation can be defined as

$$
\begin{aligned}
\underbrace{\boldsymbol{f}(\nabla \boldsymbol{v}, \boldsymbol{r})}_{\text {change due to flow }}= & \underbrace{\xi(\boldsymbol{r} \cdot \boldsymbol{r}) \frac{\boldsymbol{r r}: \boldsymbol{D}}{\boldsymbol{r} \cdot \boldsymbol{r}} \boldsymbol{r}}_{\text {segment stretching }} \\
& +\underbrace{\zeta(\boldsymbol{r} \cdot \boldsymbol{r}) \boldsymbol{r} \cdot\left(\boldsymbol{D}-\frac{\boldsymbol{r} \boldsymbol{r}: \boldsymbol{D}}{\boldsymbol{r} \cdot \boldsymbol{r}} \boldsymbol{I}+\boldsymbol{W}-\boldsymbol{R}\right)}_{\text {segment orientation }} \\
& +\underbrace{\boldsymbol{r} \cdot \boldsymbol{R}}_{\text {segment solid-body rotation }}
\end{aligned}
$$

where $\xi$ and $\zeta(0 \leq \xi, \zeta \leq 1)$ are functions of $|\boldsymbol{r}|$ that represent the resistance of the molecules to stretch and rotation and $\boldsymbol{R}$ is a dyadic representing the average over a material volume of the rate of rotation of the molecules. Hereafter, $\boldsymbol{R} \equiv \boldsymbol{W}$ (the case $\boldsymbol{R} \neq \boldsymbol{W}$ is analyzed by [60].)

According to the definition of $\mathcal{F}(\nabla \boldsymbol{v}, \boldsymbol{M}, e)$, Eqs. (173) and (177)

$$
\begin{aligned}
\mathcal{F}(\nabla \boldsymbol{v}, \boldsymbol{M}, e)= & \int_{\boldsymbol{r} \in R^{3}} \mathrm{~d} \boldsymbol{r} \Psi 2 \xi \frac{\boldsymbol{D}: \boldsymbol{r} \boldsymbol{r}}{\boldsymbol{r} \cdot \boldsymbol{r}} \boldsymbol{r} \\
& +\int_{\boldsymbol{r} \in R^{3}} \mathrm{~d} \boldsymbol{r} \Psi \zeta\left[\boldsymbol{r} \boldsymbol{r} \cdot \boldsymbol{D}+\boldsymbol{D} \cdot \boldsymbol{r} \boldsymbol{r}-2 \frac{\boldsymbol{D}: \boldsymbol{r} \boldsymbol{r}}{\boldsymbol{r} \cdot \boldsymbol{r}} \boldsymbol{r}\right] \\
& +\int_{\boldsymbol{r} \in R^{3}} \mathrm{~d} \boldsymbol{r} \Psi\left(\boldsymbol{r} \boldsymbol{r} \cdot \boldsymbol{W}+\boldsymbol{W}^{\mathrm{T}} \cdot \boldsymbol{r} \boldsymbol{r}\right) .
\end{aligned}
$$

Eq. (178) suggests approximating $\mathcal{F}(\nabla \boldsymbol{v}, \boldsymbol{M}, e)$ as

$$
\begin{aligned}
\mathcal{F}(\nabla \boldsymbol{v}, \boldsymbol{M}, e)= & 2 \xi(\overline{\boldsymbol{M}}, \bar{e}) \frac{\boldsymbol{D}: \boldsymbol{M}}{\boldsymbol{I}: \boldsymbol{M}} \boldsymbol{M} \\
& +\zeta(\overline{\boldsymbol{M}}, \bar{e})\left(\boldsymbol{M} \cdot \boldsymbol{D}+\boldsymbol{D} \cdot \boldsymbol{M}-2 \frac{\boldsymbol{D}: \boldsymbol{M}}{\boldsymbol{I}: \boldsymbol{M}} \boldsymbol{M}\right) \\
& +\boldsymbol{M} \cdot \boldsymbol{W}+\boldsymbol{W}^{\mathrm{T}} \cdot \boldsymbol{M}
\end{aligned}
$$

where $\overline{\boldsymbol{M}} \equiv 3 \boldsymbol{M} / c_{\mathrm{p}} N l^{2}, \bar{e} \equiv e / e_{0}$, and $e_{0}$ is the number of entanglements per unit mass at equilibrium. The approximations involved in passing from Eqs. (178) and (179) depend on the particular expression of $\xi$ and $\zeta$, and cannot be justified in general. Eq. (179) relies on an assumption similar to the quadratic closure approximation, except that the (unknown) constitutive functions $\xi(\boldsymbol{r})$ and $\zeta(\boldsymbol{r})$ appear inside the configurational integrals in Eq. (178). The most appropriate closure approximation for various classes of liquids is still a subject of debate, and various forms have been derived from microscopic theories as well as macroscopic arguments (e.g., [136-140]). The choice of closure approximation has quantitative and sometimes qualitative effects on the dynamics predicted by the model [141-146] — note that one of the restrictions placed on the closure approximation in [141] using the bracket approach (Eq. (18), i.e., that the closure tensor should be a homogeneous function of degree one of $\boldsymbol{M}$ ) can be derived simply by dimensional analysis.

However, Eq. (179) is a special case of Eq. (99); it has the advantage of a relatively simple form, separates the effect of polymer segment stretch and orientation at the macroscopic level, and includes all the well-known models of flow-induced conformational changes - the affine deformation model for $\xi=\zeta=1$, the Gordon-Schowalter 
'non-affine' deformation for $\xi=\zeta<1[123,124]$, the partially extending mechanism (or incomplete retraction) of Larson [106,85] for $\xi<1, \zeta=1$, and the non-extending chain deformation mechanism of Doi [96] for $\xi=0, \zeta=1$.

A function $\zeta \neq 1$ implies that the eigenvectors of the conformation dyadic do not rotate at the same angular velocity as the eigenvectors of the strain dyadic, which violates the experimentally valid (for flexible polymer melts and solutions) Lodge-Meissner relationship in step strains ([41], pp. 83-85 and 136-138). This can be avoided by choosing a slowly decreasing function $\zeta(\overline{\boldsymbol{M}})$ such that $\zeta(\overline{\boldsymbol{M}})=1$ when $\overline{\boldsymbol{M}} \approx \boldsymbol{I}$. This constraint on $\zeta(\overline{\boldsymbol{M}})$ is also consistent with the physical meaning of the function $\zeta(\overline{\boldsymbol{M}})$ because when the coils are almost unstretched they tend to follow the macroscopic deformation entirely (affinely). Similarly, when $\bar{M} \approx$ $I$ the stretching resistance should be low (unless the polymer is rod-like), $\xi(\overline{\boldsymbol{M}}) \approx 1$, so that the affine deformation formula is recovered, i.e., $\mathcal{F}(\nabla \boldsymbol{v}, \boldsymbol{M}, e)=\nabla \boldsymbol{v}^{\mathrm{T}} \cdot \boldsymbol{M}+\boldsymbol{M} \cdot \nabla \boldsymbol{v}$.

\subsubsection{Conformation changes by entropic elasticity and}

\section{Brownian motion}

The rate of change of segment conformation due to entropic elasticity and Brownian motion is usually written as [12]

$$
\begin{aligned}
\boldsymbol{g}(\boldsymbol{r}, \Psi)= & -\underbrace{\zeta^{-1}(\boldsymbol{r})}_{\begin{array}{l}
\text { anisotropic } \\
\text { friction }
\end{array}} \\
& (\underbrace{\Gamma(\boldsymbol{r}) \boldsymbol{r}}_{\begin{array}{l}
\text { intramolecular } \\
\text { elastic forces }
\end{array}}+\underbrace{\frac{\partial}{\partial \boldsymbol{r}} \cdot \xi^{-1}(\boldsymbol{r}) \ln \Psi}_{\text {Brownian forces }})
\end{aligned}
$$

where $\zeta^{-1}(\boldsymbol{r})$ is a friction dyadic that represents the possibility that a segment may move preferentially along particular directions due to the presence of neighboring oriented segments $[12,97,147-149], \Gamma(\boldsymbol{r}) \equiv \partial \Phi / \partial \boldsymbol{r}$ represents the effect of intramolecular forces derived from a potential $\Phi$, i.e., elastic forces ([12] and references therein), and $\xi^{-1}(\boldsymbol{r})$ is a dyadic that accounts for the possibility of anisotropic Brownian motion, i.e., a non-Maxwellian velocity distribution $[12,97,149]$. Because $\boldsymbol{g}$ is independent of $\nabla \boldsymbol{v}, \mathcal{G}$ is also taken to be independent of it. The most general expression of $\mathcal{G}(\boldsymbol{M}, e)$ allowed by the isotropic representation theorems is then

$$
\begin{aligned}
\mathcal{G}(\boldsymbol{M}, e)= & -\frac{c_{\mathrm{p}} N l^{2}}{\lambda}\left[g_{0}(\overline{\boldsymbol{M}}, \bar{e}) \boldsymbol{I}+g_{1}(\overline{\boldsymbol{M}}, \bar{e}) \overline{\boldsymbol{M}}\right. \\
& \left.+g_{2}(\overline{\boldsymbol{M}}, \bar{e}) \overline{\boldsymbol{M}}^{2}\right],
\end{aligned}
$$

where $g_{0}, g_{1}$, and $g_{2}$ are dimensionless functions of $\overline{\boldsymbol{M}}, \bar{e}$, and the other thermodynamic state variables, $\lambda$ is a relaxation time, and the factor $-c_{\mathrm{p}} / \lambda$ has been grouped for convenience. Eq. (181) is completely general, and its only drawback is that the effects of anisotropic friction, entropic elasticity, and Brownian motion are not uncoupled.
The function $g_{1}$ is often related to the finite extensibility of polymer coils, as in the FENE-P [12] and FENE-CR [150] models. The function $g_{2}$ is sometimes associated with the idea that a polymer molecule in a concentrated solution or melt relaxes faster along the direction of mean orientation of the surrounding molecules $[149,147,97,151]$. However, a general way has not been found to separate the effects of finite extensibility and anisotropic relaxation in the coarse-grained expression of $\mathcal{G}(\boldsymbol{M})$. The form of the functions $\xi, \zeta, g_{0}, g_{1}$, and $g_{2}$ as well as the expressions of the free energy and the local rate of entropy production due to microstructural relaxation are summarized in Table 2 for several constitutive models - see also Beris and Edwards [2], Jongschaap et al. [3], Kwon and Leonov [86] for similar summaries.

\subsubsection{Conformation changes by production and destruction of entanglements}

The rate of change of conformation by formation and destruction of entanglements (Eq. (175)) is closely related to the rates of production and destruction (Eq. (168)) of entanglements because the same kernels $\Psi_{\mathrm{p}}$ and $\Psi_{\mathrm{d}}$ appear in the definition of $\mathcal{H}, e_{\mathrm{p}}$, and $e_{\mathrm{d}}$. An exact relationship between $e_{\mathrm{p}}, e_{\mathrm{d}}$, and $\mathcal{H}$ can be derived if particularly simple, yet physically meaningful, expressions are chosen for $\Psi_{\mathrm{p}}$ and $\Psi_{\mathrm{d}}$, for example, $\Psi_{\mathrm{p}} \equiv k_{\mathrm{p}}(T, v, \boldsymbol{M}, e, \boldsymbol{D}) \Psi^{0}$ and $\Psi_{\mathrm{d}} \equiv$ $k_{\mathrm{d}}(T, v, \boldsymbol{M}, e, \boldsymbol{D}) \Psi$. This expression of the rate of production of entanglements states that entanglement generation is a random process that may progress at a rate that depends on the local average thermodynamic variables, and that the segments created when new entanglements form are distributed according to the equilibrium distribution of segment stretch and orientation $\Psi_{0}$. Similarly, the expression of the rate of destruction of entanglements accounts for possible non-linear effects only through the zeroth and second moment of the distribution function, and not the distribution function itself; the segments lost to disentanglements have the same distribution $\Psi$ of those that are left, i.e., the likelihood that a segment disappears due to disentanglement is independent of the stretch and orientation of the segment itself. The definitions in Eq. (168) lead to

$$
\begin{aligned}
e_{\mathrm{p}} & \equiv \frac{1}{2} \int_{\boldsymbol{r} \in R^{3}} \mathrm{~d} \boldsymbol{r} k_{\mathrm{p}} \Psi^{0}=\frac{1}{2} k_{\mathrm{p}} \int_{\boldsymbol{r} \in R^{3}} \mathrm{~d} \boldsymbol{r} \Psi^{0}=\frac{1}{2} k_{\mathrm{p}} c_{\mathrm{p}}^{0} \\
& =\frac{1}{2} k_{\mathrm{p}}\left(2 e^{0}+c_{\mathrm{m}}\right) \\
e_{\mathrm{d}} & \equiv \frac{1}{2} \int_{\boldsymbol{r} \in R^{3}} \mathrm{~d} \boldsymbol{r} k_{\mathrm{d}} \Psi=\frac{1}{2} k_{\mathrm{d}} \int_{\boldsymbol{r} \in R^{3}} \mathrm{~d} \boldsymbol{r} \Psi=\frac{1}{2} k_{\mathrm{d}} c_{\mathrm{p}} \\
& =\frac{1}{2} k_{\mathrm{d}}\left(2 e+c_{\mathrm{m}}\right)
\end{aligned}
$$

and the rate of production and destruction of conformation associated with these processes is 
Table 2

Constitutive functions for several models of polymer dynamics: upper-convected Maxwell/Oldroyd-B [157]; Leonov [37]; Johnson and Segalman [82]; PTT-1 and PTT-2 [83,84]; Doi (dilute and semidilute rods); Giesekus [97]; Larson [85] (the parameter $\bar{\zeta}$ in "Larson-1" is one-third the parameter $\xi$ in Larson's original paper; the parameter $\bar{\xi}$ in "Larson-2-4" coincides with the parameter $1-\xi$ in Larson's original paper); note that $I_{\boldsymbol{M}}+\boldsymbol{I}: \boldsymbol{M}^{-1}-6=$ $\boldsymbol{M}^{-1}:(\boldsymbol{M}-\boldsymbol{I})^{2}>0$; FENE-P [12]; FENE-CR [150]; Marrucci [158]. $I_{\boldsymbol{M}}, I_{\boldsymbol{M}}$, and $I I_{\boldsymbol{M}}$ are the first (trace), second, and third (determinant) invariants of $\boldsymbol{M}$. Notes: In Doi's [96] equation, the derivatives of the free energy are constrained derivatives because the $I_{M} \equiv 3$ in the model and the derivatives with respect to $\boldsymbol{M}$ must be taken by accounting for the constraint (see Appendix A). There are four versions of Larson's equation because of the approximations in Larson's original article. Larson-1 is equivalent to Eq. (54b) of Larson [85] (see the analysis in [80]); Larson-2 is equivalent to Eq. (54a) of the original paper; Larson-3 uses the same free energy and the same form of the relaxation terms of conformation as the original paper; Larson-4 uses the same free energy and the postulate of the original paper that the relaxation rate should be proportional to the stress

\begin{tabular}{|c|c|c|c|c|c|c|c|}
\hline Constitutive model & $\xi$ & $\zeta$ & $g_{0}$ & $g_{1}$ & $g_{2}$ & $(2 \rho / G) a(\boldsymbol{M})$ & $(2 \rho \lambda / G) T s_{\mathrm{g}}$ \\
\hline UCM/Oldroyd-B & 1 & 1 & -1 & 1 & 0 & $I_{M}-3$ & $I_{M}-3$ \\
\hline Leonov & 1 & 1 & $-1 / 2$ & $\left(I I_{M}-I_{M}\right) / 6$ & $1 / 2$ & $I_{M}-3$ & $I_{M}^{2} / 3+\left(I_{M} / 6-1\right) I I_{M}-3 / 2$ \\
\hline Johnson-Segalman & $\Xi$ & $\Xi$ & -1 & 1 & 0 & $\left(I_{M}-3\right) / E$ & $\left(I_{M}-3\right) / \Xi$ \\
\hline PTT-1 & $\Xi$ & $\Xi$ & $-1-\alpha\left(I_{M}-3\right)$ & $1+\alpha\left(I_{M}-3\right)$ & 0 & $\left(I_{M}-3\right) / E$ & $\left(1+\alpha\left(I_{M}-3\right)\right)\left(I_{M}-3\right) / \Xi$ \\
\hline PTT-2 & $\Xi$ & $\Xi$ & $-\exp \left(\alpha\left(I_{M}-3\right)\right)$ & $\exp \left(\alpha\left(I_{M}-3\right)\right)$ & 0 & $\left(I_{M}-3\right) / E$ & $\exp \left(\alpha\left(I_{M}-3\right)\right)\left(I_{M}-3\right) / \Xi$ \\
\hline Doi (rods) & 0 & 1 & -1 & 1 & 0 & $-\ln I I I_{M}$ & $\boldsymbol{I}: \boldsymbol{M}^{-1}-3$ \\
\hline Giesekus & 1 & 1 & $\alpha-1$ & $1-2 \alpha$ & $\alpha$ & $I_{M}-3$ & $I_{\boldsymbol{M}}-3+\alpha \boldsymbol{I}:(\boldsymbol{M}-\boldsymbol{I})^{2}$ \\
\hline Larson-1 & 1 & 1 & $-1-\bar{\zeta}\left(I_{M}-3\right)$ & $1+\bar{\zeta}\left(I_{M}-3\right)$ & 0 & $\bar{\zeta}^{-1} \ln \left(1+\bar{\zeta}\left(I_{M}-3\right)\right)$ & $I_{M}-3$ \\
\hline Larson-2 & $\underline{\bar{\xi}}$ & 1 & -1 & 1 & 0 & $\left(I_{M}-3\right) / \bar{\xi}$ & $\left(I_{M}-3\right) / \bar{\xi}$ \\
\hline Larson-3 & $\bar{\xi}$ & 1 & -1 & 1 & 0 & $I_{M}-I I I_{M}$ & $I_{\underline{M}}+I: M_{-}^{-1}-6$ \\
\hline Larson-4 & $\bar{\xi}$ & 1 & $-\bar{\xi}-3(1-\bar{\xi}) / I_{M}$ & $\bar{\xi}+3(1-\bar{\xi}) / I_{M}$ & 0 & $I_{M}-I I I_{M}$ & $\left(\bar{\xi}+3(1-\bar{\xi}) / I_{M}\right)\left(I_{M}+I: M^{-1}-6\right)$ \\
\hline FENE-P & 1 & 1 & -1 & $b-1 / b-I_{M} / 3$ & 0 & $3(b-1) \ln b-1 / b-I_{M} / 3$ & $b-1 / b-I_{M} / 3\left(b-1 / b-I_{M} / 3 I_{M}-3\right)$ \\
\hline FENE-CR & 1 & 1 & $-b-1 / b-I_{M} / 3$ & $b-1 / b-I_{M} / 3$ & 0 & $3(b-1) \ln b-1 / b-I_{M} / 3$ & $\left(b-1 / b-I_{M} / 3\right)^{2}\left(I_{M}-3\right)$ \\
\hline Marrucci & 1 & 1 & $-1 / 1-\beta\left(I_{M}-3\right)$ & $1 / 1-\beta\left(I_{M}-3\right)$ & 0 & $I_{M}-3$ & $I_{M}-3 / 1-\beta\left(I_{M}-3\right)$ \\
\hline
\end{tabular}

$$
\begin{aligned}
\mathcal{H}^{\mathrm{p}} & \equiv \int_{\boldsymbol{r} \in R^{3}} d \boldsymbol{r} k_{\mathrm{p}} \Psi^{0} \boldsymbol{r} \boldsymbol{r}=k_{\mathrm{p}} \int_{\boldsymbol{r} \in R^{3}} \mathrm{~d} \boldsymbol{r} \Psi^{0} \boldsymbol{r} \boldsymbol{r}=k_{\mathrm{p}} \boldsymbol{M}^{0} \\
& =\frac{1}{3} k_{\mathrm{p}} c_{\mathrm{p}}^{0} N l^{2} \boldsymbol{I}=k_{\mathrm{p}}\left(2 e^{0}+c_{\mathrm{m}}\right) \tilde{\boldsymbol{M}}^{0}=2 e_{\mathrm{p}} \tilde{\boldsymbol{M}}^{0}
\end{aligned}
$$

$$
\begin{aligned}
\mathcal{H}^{\mathrm{d}} & \equiv \int_{\boldsymbol{r} \in R^{3}} d \boldsymbol{r} k_{\mathrm{d}} \Psi \boldsymbol{r} \boldsymbol{r}=k_{\mathrm{d}} \int_{\boldsymbol{r} \in R^{3}} \mathrm{~d} \boldsymbol{r} \Psi \boldsymbol{r} \boldsymbol{r}=k_{\mathrm{d}} \boldsymbol{M} \\
& =k_{\mathrm{d}} c_{\mathrm{p}} \tilde{\boldsymbol{M}}=k_{\mathrm{p}}\left(2 e+c_{\mathrm{m}}\right) \tilde{\boldsymbol{M}}=2 e_{\mathrm{d}} \tilde{\boldsymbol{M}},
\end{aligned}
$$

where $\tilde{\boldsymbol{M}}^{0} \equiv N l^{2} \boldsymbol{I} / 3$ and $\tilde{\boldsymbol{M}} \equiv \boldsymbol{M} / c_{\mathrm{p}}$ are respectively the average segment conformation at equilibrium and during flow. Eqs. (184) and (185) suggests the definition

$\mathcal{H} \equiv 2 e_{\mathrm{p}} N l^{2} \overline{\boldsymbol{M}}^{\mathrm{p}}-2 e_{\mathrm{d}} N l^{2} \overline{\boldsymbol{M}}^{\mathrm{d}}$

where the dyadics $\overline{\boldsymbol{M}}^{\mathrm{p}}$ and $\overline{\boldsymbol{M}}^{\mathrm{d}}$ represent the average conformation of new segments that are created by entanglement formation and existing segments that disappear by entanglement destruction. The isotropic representation theorems lead to the final expression

$$
\begin{aligned}
\mathcal{H} \equiv & 2 e_{\mathrm{p}} N l^{2}\left(k_{0}^{\mathrm{p}} \boldsymbol{I}+k_{1}^{\mathrm{p}} \overline{\boldsymbol{M}}+k_{2}^{\mathrm{p}} \overline{\boldsymbol{M}}^{2}\right) \\
& -2 e_{\mathrm{d}} N l^{2}\left(k_{0}^{\mathrm{d}} \boldsymbol{I}+k_{1}^{\mathrm{d}} \overline{\boldsymbol{M}}+k_{2}^{\mathrm{d}} \overline{\boldsymbol{M}}^{2}\right)
\end{aligned}
$$

where $k_{0}^{\mathrm{p}}, \ldots, k_{2}^{\mathrm{d}}$ are dimensionless scalar functions that may depend on the invariants of $\overline{\boldsymbol{M}}, e / e^{0}$, and the other thermodynamic properties. Eq. (187) is merely a reasonable postulate founded on the similarity of the expressions of the rate of generation of entanglements and the rate of generation of conformation due to entanglement formation and destruction.

\subsection{Relationship to reptation-based models}

The theory developed here to describe temporary networks with variable (in space and time) number of junctions (entanglements) can be related to simple descriptions of reptation such as those of Larson [85] and Jongschaap [39]. Reptation is based on introducing a distribution function $f(s, t, \boldsymbol{r}, \boldsymbol{x})$, where $s$ is arclength along a tube that constrains segments at location in space $\boldsymbol{x}$. The quantity $f(s, t, \boldsymbol{r}, \boldsymbol{x}) \mathrm{d} s$ is the number of strands with stretch and orientation $\boldsymbol{r}$ at arclength location between $s$ and $s+\mathrm{d} s$ and is related to the distribution $\Psi(t, \boldsymbol{r}, \boldsymbol{x})$ by

$\Psi(t, \boldsymbol{r}, \boldsymbol{x})=\int_{-L / 2}^{L / 2} \mathrm{~d} s f(s, t, \boldsymbol{r}, \boldsymbol{x})$

where $\pm L / 2$ are the extremities of the tube. Of course, $f$ obeys a continuity equation

$\frac{\partial f}{\partial t}+\boldsymbol{v} \cdot \nabla f+\frac{\partial}{\partial \boldsymbol{r}} \cdot f \dot{\boldsymbol{r}}+\frac{\partial}{\partial s} f \dot{s}=0$

where $\dot{s}$ is the arclength velocity with which a segment migrates along the chain, which is assumed to be due to diffusive processes, $\dot{s}=-D_{\text {rep }} \partial f / \partial s$. Integrating Eq. (189) along the length of the tube yields

$$
\frac{\partial \Psi}{\partial t}+\boldsymbol{v} \cdot \nabla \Psi+\frac{\partial}{\partial \boldsymbol{r}} \cdot \Psi \dot{\boldsymbol{r}}=-\int_{-L / 2}^{L / 2} \frac{\partial}{\partial s} f \dot{s}=-[f \dot{s}]_{-L / 2}^{L / 2}
$$

where the last term in the equation is the flux of segments into (or out of) the tube. Defining this flux as the sum of positive (entering segments) and negative (exiting segments) 
contributions $-[f \dot{s}]^{L / 2}=[f \dot{s}]_{-L / 2} \equiv(1 / 2)\left(\Psi_{\text {in }}-\Psi_{\text {out }}\right)$ yields

$\frac{\partial \Psi}{\partial t}+\boldsymbol{v} \cdot \nabla \Psi+\frac{\partial}{\partial \boldsymbol{r}} \cdot \Psi \dot{\boldsymbol{r}}=\Psi_{\text {in }}-\Psi_{\text {out }}$,

which has the same form as Eq. (163): the rate of generation of entanglements parallels the rate at which segments enter the tube; the rate of destruction of entanglements parallels the rate at which segments exit the tube. Therefore, it seems that the same analysis holds at the coarse-grained level both for reptation-based and entanglement-based models.

\subsection{Equation of change of conformation}

Eqs. (172), (179), (181) and (187) yield the expression of the rate of generation (change) of conformation $\nu \boldsymbol{M}_{\mathrm{g}}$ due to internal processes and the balance equation of conformation (no diffusion): evolution equation of conformation can be important if the three-dimensional stability of a two-dimensional flow is investigated, as is frequently desirable in the analysis of process flows of polymer solutions, because it implies that a strong planar extensional flow will induce a spanwise deformation (usually a contraction) of the polymer molecules and thus will induce spanwise stresses (usually compressive)—see [63] for a discussion of this effect in free surface flows. This property also has important implications for the development of computational codes for planar flows, because in such flows the spanwise component of the conformation tensor must be computed together with the in-plane components. This has caused errors in previously published work (see, for example, the work of Lee et al. [152] with the FENE-P equation).

Finally, once reliable experimental data are available on the average molecular stretch in the neutral direction, this

$$
\begin{aligned}
\dot{\boldsymbol{M}} \equiv & \frac{\partial \boldsymbol{M}}{\partial t}+\boldsymbol{v} \cdot \nabla \boldsymbol{M}=v \boldsymbol{M}_{\mathrm{g}}=\underbrace{2 \xi \frac{\boldsymbol{D}: \boldsymbol{M}}{\boldsymbol{I}: \boldsymbol{M}} \boldsymbol{M}}_{\text {molecular stretching }} \\
& +\underbrace{\zeta\left(\boldsymbol{M} \cdot \boldsymbol{D}+\boldsymbol{D} \cdot \boldsymbol{M}-2 \frac{\boldsymbol{D}: \boldsymbol{M}}{\boldsymbol{I}: \boldsymbol{M}}+\boldsymbol{M} \cdot(\boldsymbol{W}-\boldsymbol{R})+(\boldsymbol{W}-\boldsymbol{R})^{\mathrm{T}} \cdot \boldsymbol{M}\right)}_{\text {molecular orientation }} \\
& +\underbrace{\boldsymbol{M} \cdot \boldsymbol{R}+\boldsymbol{R}^{\mathrm{T}} \cdot \boldsymbol{M}}_{\text {solid }- \text { body rotation }}-\frac{c_{\mathrm{p}} N l^{2}}{\lambda} \underbrace{\left(g_{0} \boldsymbol{I}+g_{1} \overline{\boldsymbol{M}}+g_{2} \overline{\boldsymbol{M}}^{2}\right)}_{\text {molecular relaxation }}+\underbrace{2 e_{\mathrm{p}} N l^{2}\left(k_{0}^{\mathrm{p}} \boldsymbol{I}+k_{1}^{\mathrm{p}} \overline{\boldsymbol{M}}+k_{2}^{\mathrm{p}} \overline{\boldsymbol{M}}^{2}\right)}_{\text {segment generation }} \\
& -\underbrace{2 e_{\mathrm{d}} N l^{2}\left(k_{0}^{\mathrm{d}} \boldsymbol{I}+k_{1}^{\mathrm{d}} \overline{\boldsymbol{M}}+k_{2}^{\mathrm{d}} \overline{\boldsymbol{M}}^{2}\right)}_{\text {segment destruction }}
\end{aligned}
$$

The dimensionless functions $\xi, \zeta, \lambda, g_{0}, g_{1}, g_{2}, e_{\mathrm{p}}, e_{\mathrm{d}}, k_{0}^{\mathrm{p}}$, $k_{1}^{\mathrm{p}}, k_{2}^{\mathrm{p}}, k_{0}^{\mathrm{d}}, k_{1}^{\mathrm{d}}$, and $k_{2}^{\mathrm{d}}$ depend on the thermodynamic state variables $s$ (or $T$ ), $v$ (if the liquid is compressible), $\overline{\boldsymbol{M}}$, and $\bar{e}$ (if the liquid is entangled). Eq. (192) holds in inertial frames as well as in rigid frames translating and rotating with respect to them ([60], for details).

\subsection{Molecular contraction along the neutral eigenvector of the velocity gradient in two-dimensional flows}

An interesting feature of the equation of change of conformation Eq. (192) is that it can predict a contraction of the "molecules" in the neutral direction in two-dimensional flows both for entangled and unentangled solutions. In an unentangled solution, the equation of change of the eigenvalue $m$ of $\overline{\boldsymbol{M}}$ associated with the eigenvector of $\overline{\boldsymbol{M}}$ aligned along the neutral direction of the flow is

$\dot{m}=(\xi-\zeta) \frac{\boldsymbol{D}: \overline{\boldsymbol{M}}}{\boldsymbol{I}: \overline{\boldsymbol{M}}} m-\frac{1}{\lambda}\left(g_{0}+g_{1} m+g_{2} m^{2}\right)$,

which always admits the steady solution $m=1$ irrespective of the rate of strain only if both conditions $\xi \equiv \zeta$ and $g_{0}+g_{1}+g_{2} \equiv 0$ are satisfied. This property of the simple test can be used to decide which class of models is most appropriate to describe each type of molecules.

\section{Constraints on generation terms}

The constraints on the form of the evolution equations and the expressions of the stress are obtained by substituting Eqs. (167), (169), (170) and (192) in Eq. (148). This leads to the local entropy production inequality ([60], for details)

$$
\begin{aligned}
& \frac{1}{\rho} \boldsymbol{\sigma}: \boldsymbol{D}+\frac{1}{\rho} \boldsymbol{\tau}: \boldsymbol{D}-\left(e_{\mathrm{p}}-e_{\mathrm{d}}\right) \epsilon \\
& \quad-2\left[(\xi-\zeta) \frac{\boldsymbol{M}}{\boldsymbol{I}: \boldsymbol{M}} \boldsymbol{M}: \boldsymbol{S}-\zeta \boldsymbol{M} \cdot \boldsymbol{S}\right]: \boldsymbol{D} \\
& \quad+\frac{c_{\mathrm{p}} N l^{2}}{\lambda}\left(g_{0} \boldsymbol{I}: \boldsymbol{S}+g_{1} \overline{\boldsymbol{M}}: \boldsymbol{S}+g_{2} \overline{\boldsymbol{M}}^{2}: \boldsymbol{S}\right) \\
& \quad-2 e_{\mathrm{p}} N l^{2} \tilde{\boldsymbol{M}}_{\mathrm{p}}: \boldsymbol{S}+2 e_{\mathrm{d}} N l^{2} \tilde{\boldsymbol{M}}_{\mathrm{d}}: \boldsymbol{S} \geq 0
\end{aligned}
$$

where $\tilde{\boldsymbol{M}}_{\mathrm{p}}$ is the average dimensionless conformation of new segments generated when entanglements form (strands enter the tube), and $\tilde{\boldsymbol{M}}_{\mathrm{d}}$ is the average dimensionless conformation of segments lost due to entanglement destruction 
(strands escaped from the tube). If Lodge's [128] simplifying hypotheses are used, then $\tilde{\boldsymbol{M}}_{\mathrm{p}}$ coincides with the mean conformation, $\tilde{\boldsymbol{M}}_{\mathrm{p}} \equiv \overline{\boldsymbol{M}}$, and $\tilde{\boldsymbol{M}}_{\mathrm{d}}$ equals the equilibrium conformation, $\tilde{\boldsymbol{M}}_{\mathrm{d}} \equiv \boldsymbol{I}$.

Some of the terms in Eq. (194) do not contribute to entropy production. Specifically, $\boldsymbol{\sigma}: \boldsymbol{D}$ is the rate of reversible working on time scales shorter than the relaxation times of the microstructural elastic-like conformation and entanglement variables; therefore, it must be balanced by the rate of change of Helmholtz free energy associated with the rate of change of conformation and entanglements due to reversible processes. The analysis of Eq. (194) in the nonlinear case is complicated by the possible nonlinear dependence of the rates of production $e_{\mathrm{p}}$ and destruction $e_{\mathrm{d}}$ of entanglements on the strain rate tensor. ${ }^{8}$ To streamline the analysis, it is convenient to split the rates of production and destruction of entanglements into strain rate independent terms, respectively $e_{\mathrm{p}}^{0}$ and $e_{\mathrm{d}}^{0}$, reversible, strain rate dependent term, $e_{\mathrm{p}}^{r D}$ and $e_{\mathrm{d}}^{r \boldsymbol{D}}$, and irreversible, strain rate dependent terms $e_{\mathrm{p}}^{i \boldsymbol{D}}$ and $e_{\mathrm{d}}^{i \boldsymbol{D}}$ :

$e_{\mathrm{p}} \equiv e_{\mathrm{p}}^{0}(\boldsymbol{M}, e)+e_{\mathrm{p}}^{r \boldsymbol{D}}(\boldsymbol{M}, e, \boldsymbol{D})+e_{\mathrm{p}}^{i \boldsymbol{D}}(\boldsymbol{M}, e, \boldsymbol{D})$

$e_{\mathrm{d}} \equiv e_{\mathrm{d}}^{0}(\boldsymbol{M}, e)+e_{\mathrm{d}}^{r \boldsymbol{D}}(\boldsymbol{M}, e, \boldsymbol{D})+e_{\mathrm{d}}^{i \boldsymbol{D}}(\boldsymbol{M}, e, \boldsymbol{D})$.

The functions $e_{\mathrm{p}}^{0}$ and $e_{\mathrm{d}}^{0}$ represent entanglement formation and destruction independent of flow. They account for spontaneous microstructural rearrangements due to the non-equilibrium conformation of the polymer segments and of their degree of entanglement. An additional principle is needed to split the deformation-dependent entanglement formation and destruction into reversible and irreversible parts: the entropy production rate is an even function of the rate of strain,

$s_{\mathrm{g}}(T, v, \boldsymbol{M}, e, \boldsymbol{D})=s_{\mathrm{g}}(T, v, \boldsymbol{M}, e,-\boldsymbol{D})$

More general forms of this principle were recorded by Woods ([113], p. 157) and Jongschaap et al. [3]. Eq. (197) implies that the terms in Eq. (194) that change sign when the sign of $\boldsymbol{D}$ is reversed do not contribute to entropy production and must add to zero. Because $e_{\mathrm{p}}$ and $e_{\mathrm{d}}$ multiply functions that are independent of $\boldsymbol{D}$ in Eq. (194), the reversible part of the entanglement formation $e_{\mathrm{p}}^{r \boldsymbol{D}}$ and destruction $e_{\mathrm{d}}^{r \boldsymbol{D}}$ rates are odd functions of $\boldsymbol{D}$, whereas the irreversible parts of the entanglement formation $e_{\mathrm{p}}^{i \boldsymbol{D}}$ and destruction $e_{\mathrm{d}}^{i \boldsymbol{D}}$ are even functions of $\boldsymbol{D}$. It is useful to analyze separately the three important cases included in Eqs. (194)-(196):

${ }^{8}$ In this case, the "Grmela expression" $[3,39,91]$ for the elastic stress $\boldsymbol{\sigma}=\sum_{i} \frac{\partial a}{\partial \boldsymbol{\Theta}_{i}} \odot \frac{\partial \boldsymbol{\Theta}_{i}{ }^{g-}}{\partial \boldsymbol{D}}$

where $a$ is the Helmholtz free energy, $\boldsymbol{\Theta}_{i}$ are the internal variables, $\boldsymbol{\Theta}_{i}^{g-}$ is the reversible part of the generation term in the equation of change of $\boldsymbol{\Theta}_{i}$ and $\odot$ is an appropriate dot product does not hold because one of the $\boldsymbol{\Theta}_{i}^{g-}$ is not a linear function of $\boldsymbol{D}$.
Sec. 8.1: $e_{\mathrm{p}}, e_{\mathrm{d}}$ are independent of $\boldsymbol{D}, e_{\mathrm{p}} \equiv e_{\mathrm{p}}^{0}(\boldsymbol{M}, e)$, and $e_{\mathrm{d}} \equiv e_{\mathrm{d}}^{0}(\boldsymbol{M}, e)$. This case includes the models of Marrucci et al. [130], Acierno et al. [131], Mewis and Denn [127], and De Cleyn and Mewis [132].

Sec. 8.2: $e_{\mathrm{p}}, e_{\mathrm{d}}$ are even functions of $\boldsymbol{D}, e_{\mathrm{p}} \equiv e_{\mathrm{p}}^{0}(\boldsymbol{M}, e)+$ $e_{\mathrm{p}}^{i \boldsymbol{D}}(\boldsymbol{M}, e, \boldsymbol{D})$, and $e_{\mathrm{d}} \equiv e_{\mathrm{d}}^{0}(\boldsymbol{M}, e)+e_{\mathrm{d}}^{i \boldsymbol{D}}(\boldsymbol{M}, e, \boldsymbol{D})$, which means that entanglement formation and destruction are irreversible processes. This case includes the models of Liu et al. [133], Moldenaers and Mewis [153], and Yosick et al. [126].

Sec. 8.3: $e_{\mathrm{p}}, e_{\mathrm{d}}$ depend on $\boldsymbol{D}$, and all the terms in Eqs. (195) and (196) are non-zero. This is the most general case and it includes both reversible and irreversible mechanisms of entanglement production and destruction. None of the published models falls into this category. The general model of Öttinger [107] includes the case where the rate of change of a scalar (which could be taken as the entanglement density) consists both by an irreversible part (independent of rate of strain) and a reversible part (linear in the rate of strain).

\subsection{Strain-rate independent entanglement generation}

The local entropy production inequality Eq. (194) can be rewritten as

$$
\begin{aligned}
& \frac{1}{\rho} \boldsymbol{\tau}: \boldsymbol{D}+\left[\frac{\sigma}{\rho}-2(\xi-\zeta) \frac{\boldsymbol{M}}{\boldsymbol{I}: \boldsymbol{M}} \boldsymbol{M}: \boldsymbol{S}-2 \zeta \boldsymbol{M} \cdot \boldsymbol{S}\right]: \boldsymbol{D} \\
& \quad+\frac{c_{\mathrm{p}} N l^{2}}{\lambda}\left(g_{0} \boldsymbol{I}: \boldsymbol{S}+g_{1} \overline{\boldsymbol{M}}: \boldsymbol{S}+g_{2} \overline{\boldsymbol{M}}^{2}: \boldsymbol{S}\right) \\
& \quad-\left(e_{\mathrm{p}}^{0}-e_{\mathrm{d}}^{0}\right) \epsilon-2 e_{\mathrm{p}}^{0} N l^{2} \tilde{\boldsymbol{M}}_{\mathrm{p}}: \boldsymbol{S}+2 e_{\mathrm{d}}^{0} N l^{2} \tilde{\boldsymbol{M}}_{\mathrm{d}}: \boldsymbol{S} \geq 0,
\end{aligned}
$$

with $\boldsymbol{\tau}(-\boldsymbol{D})=-\boldsymbol{\tau}(\boldsymbol{D})$ by definition of viscous stress. Because Eq. (198) should hold for all possible values of $\boldsymbol{D}$, it follows that

$$
\underbrace{\frac{1}{\rho} \boldsymbol{\tau}: \boldsymbol{D}}_{\begin{array}{l}
\text { dissipation by } \\
\text { viscous flow }
\end{array}} \geq 0
$$

$$
\begin{aligned}
\underbrace{\boldsymbol{\sigma}}_{\text {elastic stress }}=\underbrace{2 \rho \xi \frac{\boldsymbol{M}}{\boldsymbol{I}: \boldsymbol{M}} \boldsymbol{M}: \boldsymbol{S}}_{\begin{array}{c}
\text { stress by molecular } \\
\text { stretching }
\end{array}} \\
+\underbrace{2 \rho \zeta\left(-\frac{\boldsymbol{M}}{\boldsymbol{I}: \boldsymbol{M}} \boldsymbol{M}: \boldsymbol{S}+\boldsymbol{M} \cdot \boldsymbol{S}\right)}_{\begin{array}{c}
\text { stress by molecular } \\
\text { orientation }
\end{array}}
\end{aligned}
$$




$$
\underbrace{\frac{c_{\mathrm{p}} N l^{2}}{\lambda}\left(g_{0} \boldsymbol{I}: \boldsymbol{S}+g_{1} \overline{\boldsymbol{M}}: \boldsymbol{S}+g_{2} \overline{\boldsymbol{M}}^{2}: \boldsymbol{S}\right)}_{\text {free energy drop by segment relaxation }}
$$

free energy drop by net destruction of entanglements

$$
\begin{aligned}
& \underbrace{2 e_{\mathrm{p}}^{0} N l^{2} \tilde{\boldsymbol{M}}_{\mathrm{p}}: S}_{\text {free energy rise by entanglement-driven generation of segments }} \\
& +\underbrace{2 e_{\mathrm{d}}^{0} N l^{2} \tilde{\boldsymbol{M}}_{\mathrm{d}}: S}_{\text {free energy drop by disentanglement-driven destruction of segments }} \quad \geq 0 .
\end{aligned}
$$

Eq. (199) says that viscous stress contributes to entropy production independently of molecular relaxation and entanglement. Eq. (200) says that the elastic stress is due to the stretching (terms with the prefactor $\xi$ ) and orientation due to relative rotation (terms with the prefactor $\zeta$ ) of segments induced by the rate of strain. Eq. (201) accounts for the dissipation caused by molecular relaxation and entanglement generation.

Eqs. (199)-(201) are a consequence of the balance laws, the entropy inequality, and the constitutive assumptions about $\boldsymbol{M}_{\mathrm{g}}$ and $e_{\mathrm{g}}$. One more postulate can be added to Eqs. (199)-(201). The functions $\mathcal{G}$ and $\mathcal{H}$ account for two distinct physical phenomena. The relaxation of segments $\mathcal{G}$ describes the effects of intramolecular forces and Brownian motion on segment conformation, whereas $\mathcal{H}$ accounts for losses and gains of segments due to destruction and formation of entanglements (reptation out of and into the tube). These two processes occur on the same time scale; thus the entropy produced by them collectively should be non-negative (Eq. (201)). However, the microscopic definitions of $\mathcal{G}$ (Eq. (174)) and $\mathcal{H}$ (Eq. (175)) suggest that each of these two processes produces entropy independently; therefore, it is reasonable to postulate that

$$
\begin{aligned}
& \frac{c_{\mathrm{p}} N l^{2}}{\lambda}\left(g_{0} \boldsymbol{I}: \boldsymbol{S}+g_{1} \overline{\boldsymbol{M}}: \boldsymbol{S}+g_{2} \overline{\boldsymbol{M}}^{2}: \boldsymbol{S}\right) \geq 0 \\
& \left(e_{\mathrm{d}}^{0}-e_{\mathrm{p}}^{0}\right) \epsilon-2 e_{\mathrm{p}}^{0} N l^{2} \tilde{\boldsymbol{M}}_{\mathrm{p}}: \boldsymbol{S}+2 e_{\mathrm{d}}^{0} N l^{2} \tilde{\boldsymbol{M}}_{\mathrm{d}}: \boldsymbol{S} \geq 0 .
\end{aligned}
$$

Constitutive models that violate either Eq. (202) or Eq. (203) but satisfy Eq. (201) cannot be dismissed as unrealistic because they do not violate the second law of thermodynamics. Yet they include either of the following concepts:

- entropic elasticity can drive segments to states of higher free energy by feeding on entanglement production and destruction, which simultaneously tend to drive the system to a state of lower free energy;

- entanglement production and destruction may push the system to states of higher free energy by feeding on the concurrent entropic relaxation of segments, which drive the system toward a state of lower free energy.
Whether only Eq. (201) should be satisfied or both Eqs. (202) and (203) cannot be decided by using the "equilibrium version" of the second law $\left(\dot{s}_{\mathrm{g}} \geq 0\right)$, although it seems reasonable to postulate that both inequalities should hold in "non-equilibrium" situations.

\subsection{Entanglement generation as irreversible process}

The local entropy production inequality (Eq. (194)) is

$$
\begin{aligned}
& \frac{1}{\rho} \boldsymbol{\tau}: \boldsymbol{D}+\left(e_{\mathrm{d}}^{i \boldsymbol{D}}-e_{\mathrm{p}}^{i \boldsymbol{D}}\right) \epsilon+2 e_{\mathrm{d}}^{i \boldsymbol{D}} N l^{2} \tilde{\boldsymbol{M}}_{\mathrm{d}}: \boldsymbol{S}-2 e_{\mathrm{p}}^{i \boldsymbol{D}} N l^{2} \tilde{\boldsymbol{M}}_{\mathrm{p}}: \boldsymbol{S} \\
& \quad+\left[\frac{\boldsymbol{\sigma}}{\rho}-2(\xi-\zeta) \frac{\boldsymbol{M}}{\boldsymbol{I}: \boldsymbol{M}} \boldsymbol{M}: \boldsymbol{S}-2 \zeta \boldsymbol{M} \cdot \boldsymbol{S}\right]: \boldsymbol{D} \\
& \quad+\frac{c_{\mathrm{p}} N l^{2}}{\lambda}\left(g_{0} \boldsymbol{I}: \boldsymbol{S}+g_{1} \overline{\boldsymbol{M}}: \boldsymbol{S}+g_{2} \overline{\boldsymbol{M}}^{2}: \boldsymbol{S}\right) \\
& \quad+\left(e_{\mathrm{d}}^{0}-e_{\mathrm{p}}^{0}\right) \epsilon+2 e_{\mathrm{d}}^{0} N l^{2} \tilde{\boldsymbol{M}}_{\mathrm{d}}: \boldsymbol{S}-2 e_{\mathrm{p}}^{0} N l^{2} \tilde{\boldsymbol{M}}_{\mathrm{p}}: \boldsymbol{S} \geq 0
\end{aligned}
$$

The term

$$
\left[\frac{\sigma}{\rho}-2(\xi-\zeta) \frac{\boldsymbol{M}}{\boldsymbol{I}: \boldsymbol{M}} \boldsymbol{M}: \boldsymbol{S}-2 \zeta \boldsymbol{M} \cdot \boldsymbol{S}\right]: \boldsymbol{D}
$$

is the only one that changes sign when the strain rate is reversed; therefore, it cannot contribute to dissipation and Eq. (200) holds (see Section 8.1). The first four terms in Eq. (204) depend on $\boldsymbol{D}$, whereas the last four terms are independent of $\boldsymbol{D}$; therefore, the inequalities

$$
\begin{aligned}
& \underbrace{\frac{1}{\rho} \boldsymbol{\tau}: \boldsymbol{D}}_{\text {free energy drop by viscous flow }} \\
& +\quad \underbrace{\left(e_{\mathrm{d}}^{i \boldsymbol{D}}-e_{\mathrm{p}}^{i \boldsymbol{D}}\right) \epsilon} \\
& \text { free energy drop by straining-induced net destruction of entanglements } \\
& +\quad \underbrace{2 e_{\mathrm{d}}^{i \boldsymbol{D}} N l^{2} \tilde{\boldsymbol{M}}_{\mathrm{p}}: \boldsymbol{S}} \\
& \text { free energy rise by straining-induced generation of segments } \\
& -\underbrace{2 e_{\mathrm{p}}^{i \boldsymbol{D}} N l^{2} \tilde{\boldsymbol{M}}_{\mathrm{d}}: \boldsymbol{S}}_{\text {free energy drop by straining-induced destruction of segments }} \geq 0
\end{aligned}
$$

$$
\begin{array}{r}
\underbrace{\frac{c_{\mathrm{p}} N l^{2}}{\lambda}\left(g_{0} \boldsymbol{I}: \boldsymbol{S}+g_{1} \overline{\boldsymbol{M}}: g_{2} \overline{\boldsymbol{M}}^{2}: \boldsymbol{S}\right)}_{\text {free energy drop by segment relaxation }} \\
+\quad \underbrace{\left(e_{\mathrm{d}}^{0}-e_{\mathrm{p}}^{0}\right) \frac{\partial a}{\partial e}}
\end{array}
$$

free energy drop by flow-independent net destruction of entanglements

$$
\begin{aligned}
& \underbrace{2 e_{\mathrm{d}}^{0} N l^{2} \tilde{\boldsymbol{M}}_{\mathrm{d}}: S}_{\text {free energy drop by flow-independent destruction of segments }} \\
& \underbrace{2 e_{\mathrm{p}}^{0} N l^{2} \tilde{\boldsymbol{M}}_{\mathrm{p}}: S}_{\text {free energy rise by flow-independent generation of segments }}
\end{aligned}
$$


hold independently. These inequalities follow from the balance laws, the entropy inequality, the constitutive equations of the rates of generation of entanglements and conformation, and the postulate that the entropy production rate is an even function of the rate of strain.

Additional assumptions can be made to break down Eqs. (206) and (207) further. If dissipation by viscous stresses and that by flow-induced entanglement generation are taken to be independent, then

$$
\begin{aligned}
& \frac{1}{\rho} \boldsymbol{\tau}: \boldsymbol{D} \geq 0 \\
& \left(e_{\mathrm{d}}^{i \boldsymbol{D}}-e_{\mathrm{p}}^{i \boldsymbol{D}}\right) \epsilon+2 e_{\mathrm{d}}^{i \boldsymbol{D}} N l^{2} \tilde{\boldsymbol{M}}_{\mathrm{d}}: \boldsymbol{S}-2 e_{\mathrm{p}}^{i \boldsymbol{D}} N l^{2} \tilde{\boldsymbol{M}}_{\mathrm{p}}: \boldsymbol{S} \geq 0 .
\end{aligned}
$$

Whether the inequalities Eqs. (208) and (209) should hold separately or not is an open question. However, Eq. (206) carries the interesting implication that the viscous stress may be related to the rate of generation of entanglements induced by flow.

If segment relaxation and flow-independent entanglement generation are assumed to dissipate separately (see discussion in Section 8.1) then inequalities (202) and (203) hold.

\subsection{Reversible and irreversible entanglement generation}

In this case, the local entropy production inequality (Eq. (194)) reads

$$
\begin{aligned}
& \frac{1}{\rho} \boldsymbol{\tau}: \boldsymbol{D}+\left(e_{\mathrm{d}}^{i \boldsymbol{D}}-e_{\mathrm{p}}^{i \boldsymbol{D}}\right) \epsilon+2 e_{\mathrm{d}}^{i \boldsymbol{D}} N l^{2} \tilde{\boldsymbol{M}}_{\mathrm{d}}: \boldsymbol{S}-2 e_{\mathrm{p}}^{i \boldsymbol{D}} N l^{2} \tilde{\boldsymbol{M}}_{\mathrm{p}}: \boldsymbol{S} \\
& \quad+\left[\frac{\boldsymbol{\sigma}}{\rho}-2(\xi-\zeta) \frac{\boldsymbol{M}}{\boldsymbol{I}: \boldsymbol{M}} \boldsymbol{M}: \boldsymbol{S}-2 \zeta \boldsymbol{M} \cdot \boldsymbol{S}\right]: \boldsymbol{D} \\
& \quad+\left(e_{\mathrm{d}}^{r \boldsymbol{D}}-e_{\mathrm{p}}^{r \boldsymbol{D}}\right) \epsilon+2 e_{\mathrm{d}}^{r \boldsymbol{D}} N l^{2} \tilde{\boldsymbol{M}}_{\mathrm{d}}: \boldsymbol{S}-2 e_{\mathrm{p}}^{r \boldsymbol{D}} N l^{2} \tilde{\boldsymbol{M}}_{\mathrm{p}}: \boldsymbol{S} \\
& \quad+\frac{c_{\mathrm{p}} N l^{2}}{\lambda}\left(g_{0} \boldsymbol{I}: \boldsymbol{S}+g_{1} \overline{\boldsymbol{M}}: \boldsymbol{S}+g_{2} \overline{\boldsymbol{M}}^{2}: \boldsymbol{S}\right) \\
& \quad+\left(e_{\mathrm{d}}^{0}-e_{\mathrm{p}}^{0}\right) \epsilon+2 e_{\mathrm{d}}^{0} N l^{2} \tilde{\boldsymbol{M}}_{\mathrm{d}}: \boldsymbol{S}-2 e_{\mathrm{p}}^{0} N l^{2} \tilde{\boldsymbol{M}}_{\mathrm{d}}: \boldsymbol{S} \geq 0 .
\end{aligned}
$$

The terms that change sign when the strain rate is reversed must add to zero:

$$
\begin{aligned}
0= & {\left[\frac{\boldsymbol{\sigma}}{\rho}-2(\xi-\zeta) \frac{\boldsymbol{M}}{\boldsymbol{I}: \boldsymbol{M}} \boldsymbol{M}: \boldsymbol{S}-2 \zeta \boldsymbol{M} \cdot \boldsymbol{S}\right]: \boldsymbol{D} } \\
& +\left(e_{\mathrm{d}}^{r \boldsymbol{D}}-e_{\mathrm{p}}^{r \boldsymbol{D}}\right) \epsilon+2 e_{\mathrm{d}}^{r \boldsymbol{D}} N l^{2} \tilde{\boldsymbol{M}}_{\mathrm{d}}: \boldsymbol{S}-2 e_{\mathrm{p}}^{r \boldsymbol{D}} N l^{2} \tilde{\boldsymbol{M}}_{\mathrm{p}}: \boldsymbol{S}
\end{aligned}
$$

The reversible rates of production and destruction of entanglements must be split into a linear term and a non-linear term to obtain the relationship between elastic stress, conformation and entanglements; the two terms are defined by

$$
\begin{aligned}
& e_{\mathrm{p}}^{r \boldsymbol{D}} \equiv \boldsymbol{R}_{\mathrm{p}}^{e \boldsymbol{v}}: \boldsymbol{D}+e_{\mathrm{p}}^{r N L} \\
& e_{\mathrm{d}}^{r \boldsymbol{D}} \equiv \boldsymbol{R}_{\mathrm{d}}^{e \boldsymbol{v}}: \boldsymbol{D}+e_{\mathrm{d}}^{r N L}
\end{aligned}
$$

where $\boldsymbol{R}_{\mathrm{p}}^{e v}$ and $\boldsymbol{R}_{\mathrm{d}}^{e v}$ may depend on the state variables but are independent of $\boldsymbol{D}$, and $e_{\mathrm{p}}^{r N L}, e_{\mathrm{d}}^{r N L}$ are non-linear, odd functions of $\boldsymbol{D}$. Eq. (211) then becomes

$$
\begin{aligned}
0= & {\left[\frac{\boldsymbol{\sigma}}{\rho}-2(\xi-\zeta) \frac{\boldsymbol{M}}{\boldsymbol{I}: \boldsymbol{M}} \boldsymbol{M}: \boldsymbol{S}-2 \zeta \boldsymbol{M} \cdot \boldsymbol{S}\right.} \\
& +\epsilon\left(\boldsymbol{R}_{\mathrm{d}}^{e \boldsymbol{v}}-\boldsymbol{R}_{\mathrm{p}}^{e \boldsymbol{v}}\right)+2 N l^{2} \boldsymbol{R}_{\mathrm{d}}^{e \boldsymbol{v}} \tilde{\boldsymbol{M}}_{\mathrm{d}}: \boldsymbol{S} \\
& \left.-2 N l^{2} \boldsymbol{R}_{\mathrm{p}}^{e \boldsymbol{v}} \tilde{\boldsymbol{M}}_{\mathrm{p}}: \boldsymbol{S}\right]: \boldsymbol{D}+\left(e_{\mathrm{d}}^{r N L}-e_{\mathrm{p}}^{r N L}\right) \epsilon \\
& +2 e_{\mathrm{d}}^{r N L} N l^{2} \tilde{\boldsymbol{M}}_{\mathrm{d}}: \boldsymbol{S}-2 e_{\mathrm{p}}^{r N L} N l^{2} \tilde{\boldsymbol{M}}_{\mathrm{p}}: \boldsymbol{S} .
\end{aligned}
$$

Eq. (214) is of the form $\boldsymbol{A}: \boldsymbol{D}+f(\boldsymbol{D})=0$, where $f(\boldsymbol{D})$ is a nonlinear function, and must hold for any traceless, symmetric $\boldsymbol{D}$; therefore, $\boldsymbol{A}: \boldsymbol{D}=0$ and $f(\boldsymbol{D})=0$ separately. It follows that the relationship between elastic stress, conformation, and entanglements is

$$
\begin{aligned}
\underbrace{\boldsymbol{\sigma}}_{\text {elastic stress }}= & \underbrace{2 \rho \xi \frac{\boldsymbol{M}}{\boldsymbol{I}: \boldsymbol{M}}: \boldsymbol{S}}_{\text {stress by molecular stretching }} \\
& +\underbrace{2 \rho \zeta\left(-\frac{\boldsymbol{M}}{\boldsymbol{I}: \boldsymbol{M}} \boldsymbol{M}: \boldsymbol{S} \cdot \boldsymbol{M}\right)}_{\text {stress by molecular orientation }} \\
& +\underbrace{\rho \epsilon\left(\boldsymbol{R}_{\mathrm{p}}^{e v}-\boldsymbol{R}_{\mathrm{d}}^{e v}\right)}_{\text {stress by net generation of entanglements }} \\
& -\underbrace{2 \rho N l^{2} \boldsymbol{R}_{\mathrm{d}}^{e v} \tilde{\boldsymbol{M}}_{\mathrm{d}}: \boldsymbol{S}}_{\text {stress by bain loss of segments by disentanglement }}
\end{aligned}
$$

up to a constitutively indeterminate isotropic term; the non-linear contributions to the reversible rate of formation and destruction of entanglements must satisfy the relationship

$0=\left(e_{\mathrm{d}}^{r N L}-e_{\mathrm{p}}^{r N L}\right) \epsilon+2 e_{\mathrm{d}}^{r N L} N l^{2} \tilde{\boldsymbol{M}}_{\mathrm{d}}: \boldsymbol{S}-2 e_{\mathrm{p}}^{r N L} N l^{2} \tilde{\boldsymbol{M}}_{\mathrm{p}}: \boldsymbol{S}$.

Eq. (215) shows that if the free energy depends on entanglements (or on the number of strands inside the tube) and the rates of formation and destruction of entanglements include a term that depends linearly on the rate of strain, then there is a reversible stress that arises from the deformation-driven entanglement and disentanglement of the molecules.

The rate of entropy production due to internal molecular processes reduces to that examined in Section 8.2; therefore, Eqs. (206) and (207) and the following discussion apply here too. The analysis in this section hinges on the same assumptions used in Sec. 8.2. The relationship between elastic stress and microstructural state variables Eq. (215) holds because the reversible work done by the elastic stress $\boldsymbol{\sigma}: \boldsymbol{D}$ depends linearly on $\boldsymbol{D}$, i.e., $\boldsymbol{\sigma}$ is independent of $\boldsymbol{D}$; therefore, 
a non-linear dependence of the rates of production and destruction of entanglements on the rate of strain cannot lead to a net reversible work.

\section{Summary and conclusions}

A new method has been developed to account for the evolving microstructure of a flowing polymeric liquid by introducing microstructural variables that are local expectation values of microscopic features. The method extends irreversible thermodynamics based on the local equilibrium hypothesis, ensuring that the coarse-grained equations of the microstructural models are compatible with the first and second laws of thermodynamics.

When applied to unentangled polymeric liquids, the formalism developed here reproduces the results of the Generalized Bracket formalism of Grmela and Carreau [1] and Beris and Edwards [2], and of the Matrix Model of Jongschaap et al. [3]. But it appears simpler than both the Generalized Bracket and the Matrix Model. Moreover, it is more general than the thermodynamic framework of Leonov $[37,64]$, because it specifies how to introduce as many microstructural variables as needed, and because it clarifies the relationship between the elastic stress and the microstructural variables (which is assumed in Leonov's work and derived here). When applied to polymer solutions undergoing coupled flow and heat or mass transfer, some differences appear between the equations derived here and those derived by the Generalized Bracket [2] — namely, that a gradient of temperature can drive a diffusive flux of conformation (and vice versa), or that a gradient of chemical potential can drive a flux of conformation (and vice versa); however, these differences disappear if appropriate expressions for the generating functionals are assumed in the Generalized Bracket [119]; therefore, the simple thermodynamic approach and the Generalized Bracket formalism seem to yield consistent results. When applied to entangled polymeric liquids, the formalism presented here reproduces a key result obtained with GENERIC [107] - that if the rate of generation of entanglements is coupled linearly to the rate of strain, then a part of the elastic (reversible) stress is related to this coupling term.

To model flowing polymer solutions and melts, the microstructural variables of choice are the conformation tensor, representing the local expectation value of the stretch and orientation of polymer segments (which are polymer molecules in unentangled liquids, or parts of polymer molecules included between consecutive entanglements on the same chain in entangled liquids), and the number of entanglements per unit volume. A set of thermodynamic restrictions is derived on the equations of change of entanglement density and conformation, and the relationship is explained between these equations of change and the elastic stress. The equations of the theory include many previously published models of polymer behavior (or constitutive equations), some of which include an explicit entanglement variable. The thermodynamic framework is advantageous for computational modeling; the calculation of separate constitutive functions (see Table 2) and their derivatives can be delegated to subroutines or objects in a computational code; thus, changing constitutive equations, adding new ones, or mixing parts of one equation with another become straightforward, all of this while retaining the power of Newton's Method and arclength continuation. Yet, caution must be exercised when mixing models because the range of admissible values of the invariants of the conformation tensor depends on the choice of model, and the local rate of entropy production in turn depends on the instantaneous value of the invariants of the conformation tensor; thus, a proof must be sought that the entropy production rate is non-negative in the admissible range of conformation values or, alternatively, the entropy production rate must be monitored during calculations.

However, thermodynamics (in whatever embodiment) cannot provide the constitutive form of the diffusive fluxes and generation terms that appear in the transport equations of microstructure, but only a set of relationships between such terms. To arrive at a predictive set of equations guidance will be needed from kinetic theory and statistical mechanics and from insightful experiments that in the near future may be able to separate the effects of flow on molecular stretch and orientation, entanglement density, and elastic and viscous stress.

To this extent, a multi-level modeling approach may prove highly effective: the same flow could be modeled in a few selected cases with both a fine-grained model free (or nearly so) of adjustable parameters and a coarse-grained model. Developing an automatic estimation algorithm seems possible that would tune the unknown constitutive parameters and functions of the coarse-grained model to best reproduce the predictions obtained with the fine-grained model, thereby achieving the goal of projecting effectively the multitude of degrees of freedom of the fine-grained model onto a relatively small set of variables characteristic of the coarse-grained model, which could then be used to model efficiently complex process flows.

\section{Acknowledgements}

Matteo Pasquali gratefully acknowledges useful discussions and correspondence with Miroslav Grmela, Antony Beris, Rob Jongschaap, Brian Edwards, and Hans Christian Öttinger. Thanks are due also to Giulio Sarti and to the late Gianni Astarita for discussions of this work during early stages of development. Dave Morse, Chris Macosko, Lyudmila Pekurovsky, Mikhail Pekurovsky, Costas Aggelidis, Roger Fosdick, Richard James, and Dan Joseph provided helpful feedback. This work was supported by NSF and industrial sponsors through the Center for Interfacial Engineering at the University of Minnesota and by a subsequent grant from NSF to M.P. (award CTS-CAREER-0134389). 


\section{Appendix A. Derivative of a scalar function of a second-order tensor}

The formulae for computing the derivatives of a scalar isotropic function $a(\boldsymbol{A})$ with respect to a second-order tensor $\boldsymbol{A}$ are reported here for convenience; they can also be found, for example, in Truesdell and Noll [118] and Wang and Truesdell [154]. Because of isotropy, $a$ depends only on the invariants of $\boldsymbol{A}$, i.e., $a(\boldsymbol{A}) \equiv a(I, I I, I I I)$; Thus,

$\frac{\partial a}{\partial \boldsymbol{A}}=\frac{\partial a}{\partial I} \frac{\partial I}{\partial \boldsymbol{A}}+\frac{\partial a}{\partial I I} \frac{\partial I I}{\partial \boldsymbol{A}}+\frac{\partial a}{\partial I I I} \frac{\partial I I I}{\partial \boldsymbol{A}}$

where

$\frac{\partial I}{\partial \boldsymbol{A}}=\boldsymbol{I} ; \quad \frac{\partial I I}{\partial \boldsymbol{A}}=I \boldsymbol{I}-\boldsymbol{A}^{\mathrm{T}} ; \quad \frac{\partial I I I}{\partial \boldsymbol{A}}=I I I \boldsymbol{A}^{-\mathrm{T}}$

where $\boldsymbol{A}^{-\mathrm{T}}$ denotes the transpose of the inverse of $\boldsymbol{A}$. Combining the two expressions above yields

$\frac{\partial a}{\partial \boldsymbol{A}}=\left(\frac{\partial a}{\partial I}+I \frac{\partial a}{\partial I I}\right) \boldsymbol{I}-\frac{\partial a}{\partial I I} \boldsymbol{A}^{\mathrm{T}}+I I I \frac{\partial a}{\partial I I I} \boldsymbol{A}^{-\mathrm{T}}$.

If one of the invariants of the tensor is constrained to be constant, then the derivative has to be taken subject to the appropriate constraint. If the trace of $\boldsymbol{A}$ is constant, as in the rod model [96], then the constrained derivative (denoted by a $\hat{\partial})$ is

$\frac{\hat{\partial} a}{\hat{\partial} \boldsymbol{A}}=\frac{\partial a}{\partial \boldsymbol{A}}-\frac{1}{3} \frac{\partial a}{\partial \boldsymbol{A}}: \boldsymbol{I I}$.

If the determinant of $\boldsymbol{A}$ is constant, then the constrained derivative (denoted by a $\tilde{\partial}$ ) is

$\frac{\tilde{\partial} a}{\tilde{\partial} \boldsymbol{A}}=\frac{\partial a}{\partial \boldsymbol{A}}-\frac{\partial a}{\partial \boldsymbol{A}}: \frac{\boldsymbol{A}^{-1} \boldsymbol{A}^{-\mathrm{T}}}{\left(\boldsymbol{A}^{-1}: \boldsymbol{A}^{-\mathrm{T}}\right)}$.

\section{Appendix B. Invariance of entropy production under rigid changes of frames}

The expression of the entropy production rate ordinarily should not change if the balance equations are written in a non-inertial, rigid frame, because the relative accelerations arising in accelerating frames ordinarily do not contribute to the rate of conversion of mechanical energy and internal energy, $\boldsymbol{T}: \nabla \boldsymbol{v}$ ([113], p. 160). Except in extraordinary cases, the value of the thermodynamic variables does not depend on the choice of rigid frame of reference, i.e., $\hat{u}=u, \hat{s}=$ $s, \hat{v}=v, \hat{\boldsymbol{M}}=\boldsymbol{M}, \hat{e}=e$, where $(\wedge)$ indicates the value of a variable in the non-inertial frame. The vorticity $\boldsymbol{W}$ is the only quantity in Eq. (95) that changes in a rotating frame, $\hat{\boldsymbol{W}}=\boldsymbol{W}+\boldsymbol{\Omega}$, where $\boldsymbol{\Omega}$ is the angular velocity of the rotating frame with respect to the inertial frame and $\boldsymbol{\Omega}=-\boldsymbol{\Omega}^{\mathrm{T}}$. The entropy production rate in the rotating frame is

$T \hat{s}_{\mathrm{g}}=T s_{\mathrm{g}}+\frac{\partial u}{\partial \boldsymbol{M}}: \boldsymbol{R}^{\boldsymbol{M W}}: \boldsymbol{\Omega}$ therefore the requirement that the entropy production rate be the same in inertial and non-inertial frames demands that

$\frac{\partial u}{\partial \boldsymbol{M}}: \boldsymbol{R}^{\boldsymbol{M W}}: \boldsymbol{\Omega}=0$

for any skew-symmetric dyadic $\boldsymbol{\Omega}$. This condition is the same as Eq. (104), which was derived from the symmetry of the stress dyadic and is always satisfied if $\boldsymbol{R}^{\boldsymbol{M} W}$ is an isotropic function of $\boldsymbol{M}$ with $\boldsymbol{R}_{i j k l}^{\boldsymbol{M W}}=\boldsymbol{R}_{j i k l}^{\boldsymbol{M W}}=-\boldsymbol{R}_{i j l k}^{\boldsymbol{M W}}$. However, the symmetry of the stress tensor and the requirement that the entropy production rate be independent of frame are independent conditions; the first is not always true $[155,156]$, the latter is a widely accepted postulate.

\section{Appendix C. Invariance of transport equations of microstructure under rigid changes of frames}

The requirement that the transport equations of microstructure hold unchanged in any rotating rigid frame puts an additional constraint on the tensor $\boldsymbol{R}^{\boldsymbol{M W}}$. The transport equation of entanglements does not change in a rotating frame because the time derivative of a scalar does not depend on the choice of frame and the independent variables in the constitutive functions of $\boldsymbol{J}_{\mathrm{e}}, e_{\mathrm{g}}^{0}$, and $\boldsymbol{R}^{e v}$ are independent of frame. Additional terms appear in the transport equation of conformation, because the time derivative of a dyadic depends on the choice of rigid frame and so does the vorticity, and these terms must cancel because the equation holds in the rotating frame as well as the inertial frame. This gives

$\rho\left(-\boldsymbol{\Omega}^{\mathrm{T}} \cdot \boldsymbol{M}-\boldsymbol{M} \cdot \boldsymbol{\Omega}\right)=-\boldsymbol{R}^{\boldsymbol{M W}}: \boldsymbol{\Omega}$

which leads to the expression

$\boldsymbol{R}_{i j k l}^{M W}=\frac{1}{2} \rho\left(M_{i k} \delta_{j l}+M_{j k} \delta_{i l}-M_{i l} \delta_{j k}-M_{j l} \delta_{i k}\right)$.

An equivalent expression of $\boldsymbol{R}^{\boldsymbol{M W}}$ was derived by Jongschaap et al. [3].

\section{Appendix D. Non-entangled, non-isothermal, incompressible polymer solutions, uniform concentration}

The equation set describing the flow of a unentangled polymer solution is obtained from Eqs. (67)-(77) by dropping the transport equation of entanglement density and the dependence of the energy on the specific volume and entanglement density:

$$
\begin{aligned}
& 0=\nabla \cdot \boldsymbol{v} \\
& \rho \dot{\boldsymbol{v}}=\nabla \cdot \boldsymbol{T}-\nabla \Theta \\
& \rho \dot{u}=-\nabla \cdot \boldsymbol{J}_{\mathrm{u}}+\boldsymbol{T}: \boldsymbol{D}
\end{aligned}
$$


$\rho \dot{s}=-\nabla \cdot \boldsymbol{J}_{\mathrm{s}}+\frac{1}{T}\left(\boldsymbol{\tau}: \boldsymbol{D}-\nabla T \cdot \boldsymbol{J}_{\mathrm{s}}-\nabla \boldsymbol{S} \bullet \boldsymbol{J}_{\boldsymbol{M}}-\boldsymbol{S}: \boldsymbol{M}_{\mathrm{g}}^{0}\right)$

$\rho \dot{M}=-\nabla \cdot J_{M}+R^{M D}: D+R^{M W}: W+M_{\mathrm{g}}^{0}$

$\boldsymbol{T}=\boldsymbol{T}^{\mathrm{T}}=\boldsymbol{\sigma}+\boldsymbol{\tau}$

$\sigma=\sigma^{\mathrm{T}}=-\pi \boldsymbol{I}+\boldsymbol{S}: \boldsymbol{R}^{M D}$

$\tau=\tau^{T}=L^{v v}: D$

$J_{\mathrm{u}}=T J_{\mathrm{s}}+J_{M}: S$

$\boldsymbol{J}_{\mathrm{S}} \equiv-\boldsymbol{L}^{s s} \cdot \nabla T-\boldsymbol{L}^{s M} \bullet \nabla S$

$J_{M} \equiv-L^{M s} \cdot \nabla T-L^{M M} \bullet \nabla S$,

together with constitutive equations for the internal energy $u(s, \boldsymbol{M})$, the viscosity tetradic $\boldsymbol{L}^{\boldsymbol{v} v}(s, \boldsymbol{M})$, the coupling between rate of strain and conformation generation $\boldsymbol{R}^{\boldsymbol{M D}}(s, \boldsymbol{M})$, the thermal conductivity tensor $\boldsymbol{L}^{s s}(s, \boldsymbol{M})$, and the relaxation term and $\boldsymbol{M}_{\mathrm{g}}^{0}$; the coupling between vorticity and conformation generation $\boldsymbol{R}^{M W}(s, \boldsymbol{M})$ is given by Eq. (C.2).

\section{Appendix E. Non-entangled, isothermal, incompressible polymer solutions, non-uniform concentration}

The balance equations of a isothermal polymer solution in the presence of concentration gradients can be obtained from the general Eqs. (110)-(122)) by setting the density to a constant value, discarding the balance equations of internal energy and entropy (see discussion in Section 6.2), replacing the entanglement density $e$ with the polymer concentration $c_{\mathrm{p}}$, setting to zero the generation term in the equation of change of polymer concentration (stress-induced chain breakage and polymerization are not considered) and using the Helmholtz free energy $a\left(c_{\mathrm{p}}, \boldsymbol{M}\right)$ :

$0=\nabla \cdot \boldsymbol{v}$

$$
\begin{aligned}
\rho \dot{\boldsymbol{v}}= & \nabla \cdot \boldsymbol{T}-\nabla \Theta \\
= & -\nabla \pi+\nabla \cdot\left(\boldsymbol{S}: \boldsymbol{R}^{\boldsymbol{M} \boldsymbol{D}}\right)+\nabla \cdot\left(\boldsymbol{L}^{v v}: \boldsymbol{D}\right)-\nabla \Theta \\
\rho \dot{c}_{\mathrm{p}}= & -\nabla \cdot \boldsymbol{J}_{c}=\nabla \cdot\left(\boldsymbol{L}^{c c} \cdot \nabla \hat{\mu}+\boldsymbol{L}^{c \boldsymbol{M}} \bullet \nabla \boldsymbol{S}\right) \\
\rho \dot{\boldsymbol{M}}= & -\nabla \cdot \boldsymbol{J}_{\boldsymbol{M}}+\boldsymbol{R}^{\boldsymbol{M D}}: \boldsymbol{D}+\boldsymbol{R}^{\boldsymbol{M W}}: \boldsymbol{W}+\boldsymbol{M}_{\mathrm{g}}^{0} \\
= & \nabla \cdot\left(\boldsymbol{L}^{\boldsymbol{M} c} \cdot \nabla \hat{\mu}+\boldsymbol{L}^{\boldsymbol{M} \boldsymbol{M}} \bullet \nabla \boldsymbol{S}\right) \\
& +\boldsymbol{R}^{\boldsymbol{M} \boldsymbol{D}}: \boldsymbol{D}+\boldsymbol{R}^{\boldsymbol{M W}}: \boldsymbol{W}+\boldsymbol{M}_{\mathrm{g}}^{0}
\end{aligned}
$$

where the relationships between fluxes and linear phenomenological laws have been used in the right-most equalities above, and $\hat{\mu} \equiv \partial a / \partial c_{\mathrm{p}}$ and $\boldsymbol{S} \equiv \partial a / \partial \boldsymbol{M}$. This equation set is slightly different from Eqs. (9.2-6)-(9.2.8) of Beris and Edwards [2]; specifically, the gradients of the conformational affinity drive the mass and conformation fluxes in Eqs. (E.3) and (E.4), whereas gradients of elastic stress appear in the Generalized Bracket formulation; moreover, Eq. (9.2.8) is not cast in the classical convection-diffusion form (the last term is not the divergence of a flux). However, it is possible to recover Eqs. (E.3) and (E.4) by changing the form of the generating functional in the Generalized Bracket formulation [119].

\section{References}

[1] M. Grmela, P.J. Carreau, Conformation tensor rheological models, J. Non-Newtonian Fluid Mech. 23 (1987) 271-294.

[2] A.N. Beris, B.J. Edwards, Thermodynamics of Flowing Systems with Internal Microstructure, first ed., Oxford University Press, Oxford, 1994.

[3] R.J.J. Jongschaap, K.H. de Haas, C.A.J. Damen, A generic matrix representation of configuration tensor rheological models, J. Rheol. 38 (1994) 769-796.

[4] R.A. Bird, R.C. Armstrong, O. Hassager, Dynamics of Polymeric Liquids, vol. 1, second ed., Wiley, New York, 1987.

[5] C.W. Macosko, Rheology, first ed., VCH, New York, 1994.

[6] R.G. Larson, The Structure and Rheology of Complex Fluids, first ed., Oxford University Press, New York, 1999.

[7] P.J. Flory, Principles of Polymer Chemistry, first ed., Cornell University Press, Ithaca, 1953.

[8] W.W. Graessley, The entanglement concept in polymer rheology, Adv. Polym. Sci. 16 (1974) 1-179.

[9] P.G. de Gennes, Scaling Concepts in Polymer Physics, first ed., Cornell University Press, Ithaca, 1979.

[10] W.W. Graessley, Entangled linear, branched and network polymer systems-molecular theories, Adv. Polym. Sci. 47 (1982) 67117.

[11] M. Doi, S.F. Edwards, The Theory of Polymer Dynamics, first ed., Oxford University Press, Oxford, 1986.

[12] R.A. Bird, C.F. Curtiss, R.C. Armstrong, O. Hassager, Dynamics of Polymeric Liquids, vol. 2, second ed., Wiley, New York, 1987.

[13] W.W. Graessley, Polymer chain dimensions and the dependence of viscoelastic properties on concentration, molecular weight and solvent power, Polymer 21 (1980) 258-262.

[14] G. Astarita, Objective and generally applicable criteria for flow classification, J. Non-Newton. Fluid Mech. 6 (1979) 69-76.

[15] P.R. Schunk, L.E. Scriven, Constitutive equations for modeling mixed extension and shear in polymer solution processing, J. Rheol. 34 (1990) 1085-1119.

[16] R.L. Thompson, P.R.S. Mendes, Persistence of straining and flow classification, Int. J. Eng. Sci., 2004, accepted for publication.

[17] R.B. Bird, C.F. Curtiss, Nonisothermal polymeric fluids, Rheol. Acta 35 (1996) 103-109.

[18] R.B. Bird, C.F. Curtiss, Thermoviscoelasticity: continuummolecular connections, J. Non-Newtonian Fluid Mech. 79 (1998) 255-259.

[19] M. Dressler, B.J. Edwards, H.C. Öttinger, Macroscopic thermodynamics of flowing polymeric liquids, Rheol. Acta 38 (1999) 117 136.

[20] U.A. Al-Mubaiyedh, R. Sureshkumar, B. Khomami, Energetic effects on the stability of viscoelastic Dean flow, J. Non-Newtonian Fluid Mech. 95 (2000) 277-293.

[21] U.A. Al-Mubaiyedh, R. Sureshkumar, B. Khomami, The effect of viscous heating on the stability of Taylor-Couette flow, J. Fluid Mech. 462 (2002) 111-132.

[22] U.A. Al-Mubaiyedh, R. Sureshkumar, B. Khomami, Nonlinear stability analysis of viscoelastic Taylor-Couette flow in the presence of viscous heating, Phys. Fluids 14 (2002) 1056-1064. 
[23] M. Tirrell, M.F. Malone, Stress-induced diffusion of macromolecules, J. Polym. Sci. B: Polym. Phys. 15 (1977) 1569-1583.

[24] J.H. Aubert, M. Tirrell, Macromolecules in non-homogeneous velocity-gradient fields, J. Chem. Phys. 72 (1980) 2694-2701.

[25] V.G. Mavrantzas, A.N. Beris, Modeling of the rheology and flowinduced concentration changes in polymer-solutions, Phys. Rev. Lett. 69 (2) (1992) 273-276.

[26] A.N. Beris, V.G. Mavrantzas, On the compatibility between various macroscopic formalisms for the concentration and flow of dilute polymer-solutions, J. Rheol. 38 (1994) 1235-1250.

[27] C.F. Curtiss, R.B. Bird, Multicomponent diffusion in polymeric liquids, Proc. Natl. Acad. Sci. U.S.A. 93 (1996) 7440-7445.

[28] C.F. Curtiss, R.B. Bird, Diffusion-stress relations in polymer mixtures, J. Chem. Phys. 111 (1999) 10362-10370.

[29] M.V. Apostolakis, V.G. Mavrantzas, A.N. Beris, Stress gradientinduced migration effects in the Taylor-Couette flow of a dilute polymer solution, J. Non-Newtonian Fluid Mech. 102 (2002) 409445.

[30] R.M. Jendrejack, E.T. Dimalanta, D.C. Schwartz, M.D. Graham, J.J. de Pablo, DNA dynamics in a microchannel, Phys. Rev. Lett. 91 (3) (2003) 038102.

[31] B. Chitrangad, J.J.C. Picot, Similarity in orientation effects on thermal-conductivity and flow birefringence for polymers-polydimethylsiloxane, Polym. Eng. Sci. 21 (1981) 782786.

[32] D.C. Venerus, J.D. Schieber, H. Iddir, J.D. Guzman, A.W. Broerman, Relaxation of anisotropic thermal diffusivity in a polymer melt following step shear strain, Phys. Rev. Lett. 82 (1999) 366-369.

[33] H. Iddir, D.C. Venerus, J.D. Schieber, Measuring anisotropic thermal conduction in polyisobutylene following step shear strains, AIChE J. 46 (2000) 610-615.

[34] J.M. White, S.J. Muller, Viscous heating and the stability of Newtonian and viscoelastic Taylor-Couette flows, Phys. Rev. Lett. 84 (22) (2000) 5130-5133.

[35] J.M. White, S.J. Muller, The role of thermal sensitivity of fluid properties, centrifugal destabilization, and nonlinear disturbances on the viscous heating instability in Newtonian Taylor-Couette flow, Phys. Fluids 14 (11) (2002) 3880-3890.

[36] J.M. White, S.J. Muller, Experimental studies on the stability of Newtonian Taylor-Couette flow in the presence of viscous heating, J. Fluid Mech. 462 (2002) 133-159.

[37] A.I. Leonov, Nonequilibrium thermodynamics and rheology of viscoelastic polymer media, Rheol. Acta 25 (1976) 85-98.

[38] M. Grmela, Hamiltonian dynamics of incompressible elastic fluids, Phys. Lett. A 130 (1988) 355-358.

[39] R.J.J. Jongschaap, Microscopic modeling of the flow properties of polymers, Rep. Prog. Phys. 53 (1990) 1-55.

[40] M. Grmela, H.C. Öttinger, Dynamics and thermodynamics of complex fluids. I. Development of a general formalism, Phys. Rev. E 56 (1997) 6620-6632.

[41] R.G. Larson, Constitutive Equations for Polymer Melts and Solutions, first ed., Butterworths, Boston, 1988.

[42] R.B. Bird, W.E. Stewart, E.N. Lightfoot, Transport Phenomena, Wiley, New York, 1960.

[43] S.R. de Groot, P. Mazur, Non-Equilibrium Thermodynamics, first ed., North-Holland, Amsterdam, 1962.

[44] H.C. Öttinger, Stochastic Processes in Polymeric Fluids, first ed., Springer-Verlag, Berlin, 1996.

[45] R.G. Larson, T.T. Perkins, D.E. Smith, S. Chu, Hydrodynamics of a DNA molecule in a flow field, Phys. Rev. A 55 (1997) 1794-1797.

[46] R.G. Larson, H. Hu, D.E. Smith, S. Chu, Brownian dynamics simulations of a DNA molecule in an extensional flow field, J. Rheol. 43 (1999) 267-304.

[47] J.S. Hur, E.S.G. Shaqfeh, R.G. Larson, Brownian dynamics simulations of single DNA molecules in shear flow, J. Rheol. 44 (2000) $713-742$.
[48] H.P. Babcock, D.E. Smith, J.S. Hur, E.S.G. Shaqfeh, S. Chu, Relating the microscopic and macroscopic response of a polymeric fluid in a shearing flow, Phys. Rev. Lett. 85 (2000) 2018-2021.

[49] T.T. Perkins, D.E. Smith, S. Chu, Single polymer dynamics in an elongational flow, Science 276 (1997) 2016-2021.

[50] D.E. Smith, S. Chu, Response of flexible polymers to a sudden elongational flow, Science 281 (1998) 1335-1340.

[51] P.G. de Gennes, Polymer physics-molecular individualism. Science 276 (1999) 1997.

[52] R. Keunings, A survey of computational rheology, in: D.M. Binding, N.E. Hudson, J. Mewis, J.-M. Piau, C.J.S. Petrie, P. Townsend, M.H. Wagner, K. Walters (Eds.), Proceeding of the XIIIth International Congress on Rheology, vol. 1, Cambridge, UK, 2000, pp. 7-14.

[53] M.A. Hulsen, A.P.G. van Heel, B.H.A.A. van den Brule, Simulation of viscoelastic flow using Brownian configuration fields, J. NonNewtonian Fluid Mech. 70 (1997) 79-101.

[54] J. Bonvin, M. Picasso, Variance reduction methods for CONNFFESSIT-like simulations, J. Non-Newtonian Fluid Mech. 84 (1999) 191-215.

[55] C. Chauviere, A new method for micro-macro simulations of viscoelastic flows, SIAM J. Sci. Comp. 23 (2002) 2123-2140.

[56] C. Chauviere, A. Lozinski, An efficient technique for simulations of viscoelastic flows, derived from the Brownian configuration field method, SIAM J. Sci. Comp. 24 (2003) 1823-1837.

[57] M. Laso, H.C. Öttinger, Calculation of viscoelastic flow using moleculat models: the CONNFFESSIT approach, J. Non-Newtonian Fluid Mech. 47 (1993) 1-20.

[58] K. Feigl, M. Laso, H.C. Öttinger, CONNFFESSIT approach for solving a two-dimensional viscoelastic fluid problem, Macromolecules 28 (1995) 3261-3274.

[59] X. Gallez, P. Halin, G. Lielens, R. Keunings, V. Legat, The adaptive Lagrangian particle method for macroscopic and micro-macro computations of time-dependent viscoelastic flows, Comp. Methods Appl. Mech. Eng. 180 (1999) 345-364.

[60] M. Pasquali, Polymer molecules in free surface coating flows, Ph.D. thesis, University of Minnesota, Minneapolis, MN, 2000, available from UMI, Ann Arbor, MI, order number 9963019.

[61] W.J. Silliman, Viscous film flows with contact lines: finite element simulation, a basis for stability assessment and design optimization, Ph.D. thesis, University of Minnesota, Minneapolis, MN, 1979, available from UMI, Ann Arbor, MI, order number 7926172.

[62] R.I. Tanner, Engineering Rheology, second ed., Oxford University Press, Oxford, 2000.

[63] M. Pasquali, L.E. Scriven, Free surface flows of polymer solutions with models based on the conformation tensor, J. Non-Newtonian Fluid Mech. 108 (2002) 363-409.

[64] A.I. Leonov, On a class of constitutive equations for viscoelastic fluids, J. Non-Newtonian Fluid Mech. 25 (1987) 1-59.

[65] A.I. Leonov, Analysis of simple constitutive equations for viscoelastic liquids, J. Non-Newtonian Fluid Mech. 42 (1992) 323-350.

[66] M. Grmela, Bracket formulation of dissipative fluid mechanics equations, Phys. Lett. A 102 (1984) 355-358.

[67] A.N. Beris, B.J. Edwards, Poisson bracket formulation of incompressible flow equations in continuum mechanics, J. Rheol. 34 (1990) 55-78.

[68] A.N. Beris, B.J. Edwards, Poisson bracket formulation of viscoelastic flow equations of differential type: a unified approach, J. Rheol. 34 (1990) 503-538.

[69] H.C. Öttinger, M. Grmela, Dynamics and thermodynamics of complex fluids. II. Illustrations of a general formalism, Phys. Rev. E 56 (1997) 6633-6655.

[70] G.A. Maugin, R. Drouot, Internal variables and the thermodynamics of macromolecule solutions, Int. J. Eng. Sci. 21 (1983) 705-724.

[71] J. Stickforth, The rational mechanics and thermodynamics of polymeric fluids based upon the concept of a variable relaxed state, Rheol. Acta 25 (1986) 447-458. 
[72] D. Jou, J. Casas-Vàzquez, G. Lebon, Extended Irreversible Thermodynamics, first ed., Springer-Verlag, Berlin, 1993.

[73] G.A. Maugin, W. Muschik, Thermodynamics with internal variables. Part I. General concepts, J. Non-Equilib. Thermodyn. 19 (1994) 217-249.

[74] G.A. Maugin, W. Muschik, Thermodynamics with internal variables. Part II. Applications, J. Non-Equilib. Thermodyn. 19 (1994) 250289.

[75] R. Drouot, G.A. Maugin, Application of diffusive internal variables in complex fluids, J. Non-Newtonian Fluid Mech. 96 (2001) 31-43.

[76] W. Muschik, C. Papenfuss, H. Ehrentraut, A sketch of continuum thermodynamics, J. Non-Newtonian Fluid Mech. 96 (2001) 255290.

[77] I.-S. Liu, Continuum Mechanics, Springer-Verlag, New York, 2002.

[78] C. Eckart, The thermodynamics of irreversible processes. IV. The theory of elasticity and anelasticity, Phys. Rev. 73 (1948) 373-382.

[79] L.E. Malvern, Introduction to the Mechanics of a Continuous Medium, first ed., Prentice-Hall, Englewood Cliffs, NJ, 1969.

[80] A.I. Leonov, A.N. Prokunin, Nonlinear Phenomena in Flows of Viscoelastic Polymer Fluids, first ed., Chapman \& Hall, London, 1994.

[81] E.B. Wilson, Vector Analysis: A Text Book for the Use of Students of Mathematics and Physics and Founded upon the Lectures of J. Willard Gibbs, Yale University Press, New Haven, CT, 1901.

[82] M.D. Johnson, D. Segalman, A model for viscoelastic fluid behavior which allows non-affine deformation, J. Non-Newtonian Fluid Mech. 2 (1977) 225-270.

[83] N. Phan-Thien, R.I. Tanner, A new constitutive equation derived from network theory, J. Non-Newtonian Fluid Mech. 2 (1977) 353365.

[84] N. Phan-Thien, A nonlinear network viscoelastic model, J. Rheol. 22 (1978) 259-283.

[85] R.G. Larson, A constitutive equation for polymer melts based on partially extending strand convection, J. Rheol. 28 (1984) 545-571.

[86] Y. Kwon, A.I. Leonov, Stability constraints in the formulation of viscoelastic constitutive equations, J. Non-Newtonian Fluid Mech. 58 (1995) 25-46.

[87] R. Courant, D. Hilbert, Methods of Mathematical Physics, vol. 2, Wiley, New York, 1962.

[88] C. Lanczos, The Variational Principles of Mechanics, fourth ed., University of Toronto Press, Toronto, 1970.

[89] A.N. Kaufman, Dissipative hamiltonian systems, Phys. Lett. A100 (1984) 419-422.

[90] P.J. Morrison, Bracket formulation for irreversible classical fields, Phys. Lett. A100 (1984) 423-427.

[91] M. Grmela, Dependence of the stress tensor on the intramolecular viscosity, J. Rheol. 33 (1989) 207-231.

[92] I. Prigogine, C. George, F. Henin, L. Rosenfeld, A unified formulation of dynamics and thermodynamics, Chem. Scr. 4 (1973) 5-32.

[93] B.J. Edwards, A.N. Beris, Non-canonical poisson bracket for nonlinear elasticity with extensions to viscoelasticity, J. Phys. A: Math. Gen. 24 (1991) 2461-2480.

[94] H.C. Öttinger, General projection operator formalism for the dynamics and thermodynamics of complex fluids, Phys. Rev. A 57 (1998) 1416-1420.

[95] B.J. Edwards, A.N. Beris, H.C. Öttinger, An analysis of single and double generator thermodynamic formalisms for complex fluids. II. The microscopic description, J. Non-Equilib. Thermodyn. 23 (1998) 334-350.

[96] M. Doi, Molecular-dynamics and rheological properties of concentrated-solutions of rodlike polymers in isotropic and liquidcrystalline phases, J. Polym. Sci. B: Polym. Phys. 19 (1981) 229243.

[97] H. Giesekus, A simple constitutive equation for polymer fluids based on the concept of deformation-dependent tensorial mobility, J. Non-Newtonian Fluid Mech. 11 (1982) 69-109.
[98] G.A. Zevallos, M.S. Carvalho, M. Pasquali, Forward roll coating flows of viscoelastic liquids, in: A. Mammoli, C.A. Brebbia (Eds.), Moving Boundaries. VII. Computational Modelling of Free and Moving Boundary Problems, WIT Press, Southampton, UK, 2003.

[99] X. Xie, M. Pasquali, Computing 3D free surface viscoelastic flows, in: A. Mammoli, C.A. Brebbia (Eds.), Moving Boundaries. VII. Computational Modelling of Free and Moving Boundary Problems, WIT Press, Southampton, UK, 2003, pp. 225-234.

[100] X. Xie, M. Pasquali, A new, convenient way of imposing openflow boundary conditions in two- and three-dimensional viscoelastic flows, J. Non-Newtonian Fluid Mech., 2004, accepted for publication.

[101] R. Guénette, Z. Abdelmalek, A. Fortin, P. Carreau, M. Grmela, Simulation of viscoelastic flows using a conformation tensor model, J. Non-Newtonian Fluid Mech. 45 (1992) 187-208.

[102] B.J. Edwards, H.C. Öttinger, R.J.J. Jongschaap, On the relationships between thermodynamic formalisms for complex fluids, J. NonEquilib. Thermodyn. 22 (1997) 356-373.

[103] B.J. Edwards, An analysis of single and double generator thermodynamic formalisms for the macroscopic description of complex fluids, J. Non-Equilib. Thermodyn. 23 (1998) 301-333.

[104] R.J.J. Jongschaap, The matrix model, a driven state variables approach to non-equilibrium thermodynamics, J. Non-Newtonian Fluid Mech. 96 (2001) 63-76.

[105] C. Truesdell, Rational Thermodynamics, second ed., SpringerVerlag, New York, 1984.

[106] R.G. Larson, Convection and diffusion of polymer network strands, J. Non-Newtonian Fluid Mech. 13 (1983) 279-308.

[107] H.C. Öttinger, Modeling complex fluids with a tensor and a scalar as structural variables, Rev. Mex. Fisica. 48 (2002) 220-229.

[108] J.W. Gibbs, On the equilibrium of heterogeneous substances I, Trans. Conn. Acad. 3 (1875) 108-248.

[109] A.G. Fredrickson, Principles and Applications of Rheology, Prentice-Hall, Englewood Cliffs, NJ, 1964.

[110] A.S. Lodge, Elastic Liquids, Academic Press, London, 1964.

[111] F.T. Trouton, E.S. Andrews, On the viscosity of pitch-like substances, Philos. Mag. 7 (6) (1904) 347-355.

[112] G. Fano, Contributo allo studio dei corpi filanti, Archiv. Fisiol. 5 (1908) 365-370.

[113] L.C. Woods, The Thermodynamics of Fluid Systems, first ed., Oxford University Press, London, 1975.

[114] I. Müller, T. Ruggeri, Extended Thermodynamics, second ed., Springer-Verlag, New York, 1998.

[115] I.-S. Liu, Method of Lagrange multipliers for exploitation of the entropy principle, Arch. Rat. Mech. Anal. 46 (1972) 131-148.

[116] G.D.C. Kuiken, Thermodynamics of Irreversible Processes: Applications to Diffusion and Rheology, first ed., Wiley, New York, 1994.

[117] L.R.G. Treloar, The Physics of Rubber Elasticity, second ed., Oxford University Press, Oxford, 1958.

[118] C. Truesdell, W. Noll, The non-linear field theories of mechanics, in: S. Flügge (Ed.), Encyclopedia of Physics, vol. III/3, first ed., Springer-Verlag, Berlin, 1965.

[119] B.J. Edwards, Personal communication to Matteo Pasquali, 2003.

[120] C.F. Curtiss, R.B. Bird, Statistical mechanics of transport phenomena: polymeric liquid mixtures, Adv. Polym. Sci. 125 (1996) 1-101.

[121] C.F. Curtiss, R.B. Bird, Thermal conductivity of dilute solutions of chainlike polymers, J. Chem. Phys. 107 (1997) 5254-5267.

[122] F.P.T. Baaijens, Mixed finite element methods for viscoelastic flow analysis: a review, J. Non-Newtonian Fluid Mech. 79 (1998) 361385.

[123] R.J. Gordon, W.R. Schowalter, On the relation between molecular properties of polymer materials and continuum theories of fluids with structure, in: W.R. Schowalter, W.J. Minkowycz, A.V. Luikov, N.H. Afgan (Eds.), Heat and Mass Transfer in Rheologically Complex Fluids, Pergamon Press, Oxford, Prog. Heat Mass Transfer 5 (1972) 301-308. 
[124] R.J. Gordon, W.R. Schowalter, Anisotropic fluid theory: a different approach to the dumbbell theory of dilute polymer solutions, Trans. Soc. Rheol. 16 (1972) 79-97.

[125] G. Marrucci, N. Grizzuti, The free energy function of the Doi-Edwards theory: analysis of the instabilities in stress relaxation, J. Rheol. 27 (1983) 433-450.

[126] J.A. Yosick, A.J. Giacomin, P. Moldenaers, A kinetic network model for nonlinear flow behavior of molten plastics in both shear and extension, J. Non-Newtonian Fluid Mech. 70 (1997) 103-123.

[127] J. Mewis, M.M. Denn, Constitutive equations based on the transient network concept, J. Non-Newtonian Fluid Mech. 12 (1983) 6983.

[128] A.S. Lodge, A network theory of flow birefringence and stress in concentrated polymer solutions, Trans. Faraday Soc. 52 (1956) $120-130$.

[129] W.W. Graessley, Molecular entanglement theory of flow behavior in amorphous polymers, J. Chem. Phys. 43 (1965) 2696-2703.

[130] G. Marrucci, G. Titomanlio, G.C. Sarti, Testing a constitutive equation for entangled networks by elongational and shear data of polymer melts, Rheol. Acta 12 (1973) 269-275.

[131] D. Acierno, F.P. La Mantia, G. Marrucci, G. Titomanlio, A nonlinear viscoelastic model with structure-dependent relaxation times. I. Basic formulation, J. Non-Newtonian Fluid Mech. 1 (1976) 125146.

[132] G. De Cleyn, J. Mewis, A constitutive equation for polymeric liquids: application to shear flow, J. Non-Newtonian Fluid Mech. 9 (1981) 91-105.

[133] T.Y. Liu, D.S. Soong, M.C. Williams, Time-dependent rheological properties and transient structural states of entangled polymeric liquids—a kinetic network model, Polym. Eng. Sci. 21 (1981) 675687.

[134] P.G. Saffman, Vortex Dynamics, first ed., Cambridge University Press, Oxford, 1992.

[135] L.E. Wedgewood, K.R. Geurts, A non-affine network model for polymer melts, Rheol. Acta 33 (1995) 369-384.

[136] E.J. Hinch, L.G. Leal, Constitutive equations in suspension mechanics. Part 2 Approximate forms for a suspension of rigid particles affected by Brownian rotations, J. Fluid Mech. 76 (1976) 187208.

[137] G. Advani, C.L. Tucker, Closure approximation for threedimensional structure tensors, J. Rheol. 34 (1990) 367-386.

[138] C.V. Chaubal, L.G. Leal, A closure approximation for liquidcrystalline polymer models based on parametric density estimation, J. Rheol. 42 (1998) 177-201.

[139] M. Grosso, P.L. Maffettone, F. Dupret, A closure approximation for nematic liquid crystals based on the canonical distribution subspace theory, Rheol. Acta 39 (2000) 301-310.

[140] D.H. Chung, T.H. Kwon, Invariant-based optimal fitting closure approximation for the numerical prediction of flow-induced fiber orientation, J. Rheol. 46 (2002) 169-194.
[141] B.J. Edwards, H.C. Öttinger, Time-structure invariance criteria for closure approximations, Phys. Rev. E 56 (1997) 4097-4103.

[142] J. Feng, C.V. Chaubal, L.G. Leal, Closure approximations for the Doi theory: which to use in simulating complex flows of liquidcrystalline polymers? J. Rheol. 42 (1998) 1095-1119.

[143] M.G. Forest, Q. Wang, Near-equilibrium dynamics of Doi models for liquid crystal polymer flows: catastrophic and regularized behavior, J. Non-Newtonian Fluid Mech. 83 (1999) 131-150.

[144] V.K. Gupta, R. Sureshkumar, B. Khomami, J. Azaiez, Centrifugal instability of semidilute non-Brownian fiber suspensions, Phys. Fluids 14 (2002) 1958-1971.

[145] B.J. Edwards, Evaluation of the thermodynamic consistency of closure approximations in several models proposed for the description of liquid crystalline dynamics, J. Non-Equilib. Thermodyn. 27 (2002) 5-24.

[146] A.D. Rey, M.M. Denn, Dynamical phenomena in liquid-crystalline materials, Annu. Rev. Fluid Mech. 34 (2002) 233-266.

[147] H. Giesekus, Die Elastizität von Flüssigkeiten, Rheol. Acta 5 (1966) 29-35.

[148] R.B. Bird, J.R. DeAguiar, An encapsulated dumbbell model for concentrated polymer-solutions and melts. 1. Theoretical development and constitutive equation, J. Non-Newtonian Fluid Mech. 13 (1983) 149-160.

[149] R.B. Bird, J.M. Wiest, Anisotropic effects in dumbbell kinetic theory, J. Rheol. 29 (1985) 519-532.

[150] M.D. Chilcott, J.M. Rallison, Creeping flow of dilute polymer solutions past cylinders and spheres, J. Non-Newtonian Fluid Mech. 29 (1988) 381-432.

[151] J.M. Wiest, A differential constitutive equation for polymer melts, Rheol. Acta 28 (1989) 4-12.

[152] A.G. Lee, E.S.G. Shaqfeh, B. Khomami, A study of viscoelastic free surface flows by the finite element method: Hele-Shaw and slot coating flows, J. Non-Newtonian Fluid Mech. 108 (2002) 327-362.

[153] P. Moldenaers, J. Mewis, Extensional flow predictions of some differential models, in: L.A. Kleintjens, P.J. Lemstra (Eds.), Integration of Fundamental Polymer Science and Technology, Elsevier, London, 1990, pp. 351-355.

[154] C.C. Wang, C. Truesdell, Introduction to Rational Elasticity, Noordhoff, Leyden, 1973.

[155] J.S. Dahler, L.E. Scriven, Angular momentum of continua, Nature 192 (1961) 36-37.

[156] J.S. Dahler, L.E. Scriven, Theory of structured continua. I. General consideration of angular momentum and polarization, Proc. R. Soc. A 275 (1963) 504-527.

[157] J.G. Oldroyd, On the formulation of rheological equations of state, Proc. R. Soc. London A 200 (1950) 523-541.

[158] G. Marrucci, Dynamics of entanglements-a nonlinear model consistent with the cox-merz rule, J. Non-Newtonian Fluid Mech. 62 (1996) 279-289. 